\title{
Reduced Basis Kriging for Big Spatial Fields
}

\author{
Karl Pazdernik. Ranjan Maitrał Douglas Nychkał Stephan Sain ${ }^{\S}$
}

\begin{abstract}
In spatial statistics, a common method for prediction over a Gaussian random field (GRF) is maximum likelihood estimation combined with kriging. For massive data sets, kriging is computationally intensive, both in terms of CPU time and memory, and so fixed rank kriging has been proposed as a solution. The method however still involves operations on large matrices, so we develop an alteration to this method by utilizing the approximations made in fixed rank kriging combined with restricted maximum likelihood estimation and sparse matrix methodology. Experiments show that our methodology can provide additional gains in computational efficiency over fixed-rank kriging without loss of accuracy in prediction. The methodology is applied to climate data archived by the United States National Climate Data Center, with very good results.
\end{abstract}

Keywords: kriging; fixed rank kriging; Gaussian random field; sparse matrix; spatial prediction; maximum likelihood estimation; bandwidth; best linear unbiased predictor

\section{Introduction}

Data collected in a spatial domain are often incomplete. In a geostatistical setup, the spatial domain is defined as having an infinite set of locations from which a finite subset have been observed. Unobserved locations can be the result of data collected at irregularly spaced locations (such as temperature recorded only at locations with weather stations) or they may be due to a desired spatial resolution that is finer than that of the data (such as satellite images that record

\footnotetext{
${ }^{*}$ Department of Statistics, Pacific Northwest National Laboratory, Richland, Washington, USA

${ }^{\dagger}$ Department of Statistics, Iowa State University, Ames, Iowa, USA

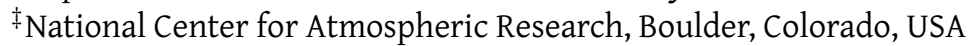

${ }^{\S}$ The Climate Corporation, San Francisco, California, USA
} 
observations in intervals of every kilometer when the desired resolution is in terms of a few meters). In either case, these unobserved locations may need to be imputed: this task is generally accomplished by a form of interpolation known as spatial prediction.

To fix ideas, consider two sets from a spatial domain $\mathcal{D}$ : one containing all the observed locations $\left(s=\left\{s_{1}, s_{2}, \ldots, s_{n}\right\} \in \mathcal{D}\right)$ and the other having the desired locations given by $\mathrm{q}\left(\boldsymbol{s}_{0}=\right.$ $\left\{\boldsymbol{s}_{01}, \boldsymbol{s}_{02}, \ldots, \boldsymbol{s}_{0 N}\right\} \in \mathcal{D}$ ), which may (or may not) include the observed set $\left\{\boldsymbol{s}_{i}: i=1,2, \ldots, n\right\}$. A random process $\boldsymbol{y}(\cdot)$ is defined on $\mathcal{D}$ by a linear combination consisting of three parts: a mean structure $\boldsymbol{x}(\cdot)$, a zero-mean spatial process $\boldsymbol{f}(\cdot)$, and a zero-mean measurement error process $\boldsymbol{\epsilon}(\cdot)$, formulated as

$$
\boldsymbol{y}(s)=x(s)+f(s)+\epsilon(s) .
$$

Kriging, one of the most common methods of spatial prediction, interpolates the missing values by utilizing spatial dependence in the prediction method. It was developed by the French mathematician Georges Matheron Matheron (1962) and named by him in honor of the empirical work by D. G. Krige, a South African mining engineer, for his work (Krige, 1951) which originated as his Masters' thesis. Specifically, in the setup of (1), the best linear unbiased predictions (BLUP) for the random variable at the desired locations $\left(\boldsymbol{y}\left(\boldsymbol{s}_{0}\right)\right)$ can be obtained under squared error loss using the first and second moments of $\boldsymbol{y}(\cdot)$ - see, e.g. Cressie (1993):

$$
\hat{\boldsymbol{y}}\left(\boldsymbol{s}_{0}\right)=\mathrm{E}\left(\boldsymbol{y}\left(\boldsymbol{s}_{0}\right)\right)+\mathcal{C}_{\boldsymbol{y}}\left(\boldsymbol{s}_{0}, \boldsymbol{s}\right) \mathcal{C}_{\boldsymbol{y}}^{-1}(\boldsymbol{s}, \boldsymbol{s})\left[\boldsymbol{y}\left(\boldsymbol{s}_{0}\right)-\mathrm{E}\left(\boldsymbol{y}\left(\boldsymbol{s}_{0}\right)\right)\right]
$$

where $\mathcal{C}_{\boldsymbol{y}}\left(\boldsymbol{s}_{0}, \boldsymbol{s}\right)=\operatorname{Cov}\left(\boldsymbol{y}\left(\boldsymbol{s}_{0}\right), \boldsymbol{y}(\boldsymbol{s})\right)$. Equation (2) is also called the kriging predictor: the methodology is extensively used in many applications, such as in mining (Richmond, 2003), hydrogeology (Chiles and Delfiner, 1999), natural sciences (Goovaerts, 1997), environmental sciences (Bayraktar and Turalioglu, 2005), remote sensing (Stein et al., 2002), or black-box modeling in computer experiments (Sacks et al., 1989). The kriging predictor involves solving an $n \times n$ linear system, which is an $O\left(n^{3}\right)$ operation: thus for datasets with large observations, kriging can be computationally impractical to implement in terms of both CPU time and memory.

There has been significant work in overcoming the computational limitations associated with kriging massive spatial fields. Most approaches can broadly be grouped into two categories. The first kind of methods use approximations to the kriging equations of (2) (Vecchia, 1988; Nychka et al., 1996; Higdon, 1998; Nychka, 2000; Nychka et al., 2002; Fuentes, 2002; Billings et al., 2002a,b; 
Stein et al., 2004; Quiñero Candela and Rasmussen, 2005; Furrer et al., 2006; Kaufman et al., 2008). These approximations include the use of orthogonal bases, covariance tapering, approximate iterative methods, inducing variables, and a reduced set of space-filling locations.

The other set of approaches in this area are concerned with choosing a class of covariance functions, within the framework of which kriging can be done exactly, regardless of the size of the data. In particular, multiresolution spatial processes can be formulated to produce covariance functions that allow for exact kriging (Huang et al., 2002; Johannesson and Cressie, 2004; Johannesson et al., 2007; Cressie and Johannesson, 2008; Banerjee et al., 2008; Finley et al., 2008; Lindgren et al., 2011; Nychka et al., 2015a). One advantage to exact kriging is that there are no concerns of how close approximate predictions and prediction errors are to their exact counterparts. However, performing estimation within the framework of a general $\boldsymbol{f}(\cdot)$ may still be computationally burdensome, so Cressie and Johannesson (2008) suggested "fixed rank kriging" which models the underlying spatial process $\boldsymbol{f}(\cdot)$ through basis functions, but allows for exact kriging. Because the methods proposed in this paper use fixed-rank kriging as a starting point, we describe it in somewhat more detail.

We continue with the setup of (1), but also assume without loss of generality that the field for $\boldsymbol{y}(\cdot)$ has mean zero. The Spatial Random Effects model (Cressie and Johannesson, 2008) uses a reduced set of independent locations $(m<n)$ coupled with a linear combination of basis functions to approximate the spatial process at the original set of locations. Specifically, let $\boldsymbol{\eta}(\cdot)$ define the spatial process at locations or knots $\boldsymbol{u}=\left\{\boldsymbol{u}_{1}, \ldots, \boldsymbol{u}_{m}\right\}$ and let $\boldsymbol{S}_{k}(\cdot)$ be the basis functions corresponding to the $k$ th knot. The spatial process is then approximated by $\boldsymbol{f}(\boldsymbol{s}) \approx$ $\sum_{k=1}^{m} \boldsymbol{S}_{k}(\boldsymbol{s}) \boldsymbol{\eta}\left(\boldsymbol{u}_{k}\right)+\boldsymbol{\delta}(\boldsymbol{s})$ and the model reduces to

$$
\boldsymbol{y}(\boldsymbol{s})=\sum_{k=1}^{m} \boldsymbol{S}_{k}(\boldsymbol{s}) \boldsymbol{\eta}\left(\boldsymbol{u}_{k}\right)+\boldsymbol{\delta}(\boldsymbol{s})+\boldsymbol{\epsilon}(\boldsymbol{s}),
$$

where $\boldsymbol{\eta}(\boldsymbol{u}) \sim N(\mathbf{0}, \boldsymbol{K})$ is mutually independent of $\boldsymbol{\delta}(\boldsymbol{s}) \sim N\left(\mathbf{0}, \sigma_{\delta}^{2} \boldsymbol{V}_{\delta}\right)$ and $\boldsymbol{\epsilon}(\boldsymbol{s}) \sim N\left(\mathbf{0}, \sigma_{\epsilon}^{2} \boldsymbol{V}_{\epsilon}\right)$ for known $\boldsymbol{V}_{\delta}$ and $\boldsymbol{V}_{\epsilon}$, both diagonal matrices with entries corresponding to the weights for the fine-scale and measurement error variances, respectively. Writing $\boldsymbol{S}$ as the $n \times m$-matrix with $k$ th column given by $\boldsymbol{S}_{k}(\boldsymbol{s})$, we get that the variance-covariance matrix of $\boldsymbol{y}(\boldsymbol{s})$ is given by $\boldsymbol{\Sigma}=$ $\boldsymbol{S} \boldsymbol{K} \boldsymbol{S}^{\prime}+\boldsymbol{D}$, where $\boldsymbol{D}=\sigma_{\delta}^{2} \boldsymbol{V}_{\delta}+\sigma_{\epsilon}^{2} \boldsymbol{V}_{\epsilon}$. With this model and by defining the particular set of basis functions between the unobserved locations and the knots as $\boldsymbol{A}=\boldsymbol{S}_{k}\left(\boldsymbol{s}_{0}\right)$ for $1 \leq k \leq m$, the 
$\log$-likelihood is $\ell\left(\boldsymbol{K}, \sigma_{\delta}^{2}, \sigma_{\epsilon}^{2} ; \boldsymbol{y}\right)=-\frac{1}{2} \boldsymbol{y}^{\prime}\left(\boldsymbol{S} \boldsymbol{K} \boldsymbol{S}^{\prime}+\boldsymbol{D}\right)^{-1} \boldsymbol{y}-\frac{1}{2} \log \left(\left|\boldsymbol{S} \boldsymbol{K} \boldsymbol{S}^{\prime}+\boldsymbol{D}\right|\right)-\frac{n}{2} \log (2 \pi)$. Assuming $\boldsymbol{s} \cap \boldsymbol{s}_{0}=\emptyset$, it follows that the kriging predictor is then $\hat{\boldsymbol{f}}\left(\boldsymbol{s}_{0}\right)=\boldsymbol{A} \hat{\boldsymbol{K}} \boldsymbol{S}^{\prime}\left(\boldsymbol{S} \hat{\boldsymbol{K}} \boldsymbol{S}^{\prime}+\hat{\boldsymbol{D}}\right)^{-1} \boldsymbol{y}$, with kriging standard error $\hat{\boldsymbol{\sigma}}_{k}\left(\boldsymbol{s}_{0}\right)=\left[\boldsymbol{A} \hat{\boldsymbol{K}} \boldsymbol{A}^{\prime}+\hat{\sigma}_{\delta}^{2} \boldsymbol{V}_{\delta}\left(\boldsymbol{s}_{0}\right)-\boldsymbol{A} \hat{\boldsymbol{K}} \boldsymbol{S}^{\prime}\left(\boldsymbol{S} \hat{\boldsymbol{K}} \boldsymbol{S}^{\prime}+\hat{\boldsymbol{D}}\right)^{-1} \boldsymbol{S} \hat{\boldsymbol{K}} \boldsymbol{A}^{\prime}\right]^{\frac{1}{2}}$, where $\hat{\boldsymbol{K}}$ and $\hat{\sigma}_{\delta}^{2}$ being the ML estimates of $\boldsymbol{K}$ and $\sigma_{\delta}^{2}$. The measurement error variance, $\sigma_{\epsilon}^{2}$, is assumed known or estimated a priori, thus, $\hat{\boldsymbol{D}}$ is completely defined given $\sigma_{\delta}^{2}$.

Cressie and Johannesson (2008) used the Sherman-Morrison-Woodbury formula (Henderson and Searle, 1981) to reduce the dimension of fixed-rank kriging by using the identity $\Sigma^{-1}=$ $\boldsymbol{D}^{-1}-\boldsymbol{D}^{-1} \boldsymbol{S}\left[\boldsymbol{K}^{-1}+\boldsymbol{S}^{\prime} \boldsymbol{D}^{-1} \boldsymbol{S}\right]^{-1} S^{\prime} \boldsymbol{D}^{-1}$, whereupon calculating $\boldsymbol{\Sigma}^{-1}$ reduces to inverting two $m \times m$ matrices as opposed to one $n \times n$ matrix. The Method of Moments was then used to obtain parameter estimates.

A likelihood-based approach was developed by Katzfuss and Cressie (2011) who adopted the expectation-maximization (EM) algorithm (Dempster et al., 1977) to maintain a positive definite covariance matrix throughout the estimation procedure. Their approach considers $\boldsymbol{y}$ as the observed data while $\boldsymbol{\eta}$ and $\boldsymbol{\delta}$ are missing. The parameter estimates are then obtained iteratively starting from appropriate initial values and updating, till convergence, via equations which update from the th to the $(t+1)$ th iteration:

$$
\begin{aligned}
\boldsymbol{K}^{(t+1)}= & \boldsymbol{K}^{(t)}-\boldsymbol{K}^{(t)} \boldsymbol{S}^{\prime} \boldsymbol{\Sigma}^{(t)^{-1}} \boldsymbol{S} \boldsymbol{K}^{(t)} \\
& +\left(\boldsymbol{K}^{(t)} \boldsymbol{S}^{\prime} \boldsymbol{\Sigma}^{(t)}{ }^{-1} \boldsymbol{y}\right)\left(\boldsymbol{K}^{(t)} \boldsymbol{S}^{\prime} \boldsymbol{\Sigma}^{(t)^{-1}} \boldsymbol{y}\right)^{\prime} \\
\sigma_{\delta}^{2(t+1)}= & \left.\sigma_{\delta}^{2(t)}+\frac{\left(\sigma_{\delta}^{2(t)}\right)^{2}}{n} \operatorname{tr}\left[\boldsymbol{\Sigma}^{(t)}\right)^{-1}\left(\boldsymbol{y} \boldsymbol{y}^{\prime} \boldsymbol{\Sigma}^{(t)^{-1}}-\boldsymbol{I}\right) \boldsymbol{V}_{\delta}\right] .
\end{aligned}
$$

Despite improvements in CPU time, implementation of this approach still requires matrix algebra involving operations on $n \times n$ matrices which can be computationally burdensome, and in some cases, prohibitive. In this paper, therefore, we propose alterations to the predetermined specifications of this method called "reduced basis kriging" that take advantage of sparse matrix operations, require less computer memory, result in faster estimation, and maintain or reduce mean square prediction error.

This paper is organized as follows. Section 2 illustrates how a QR-decomposition (Higham, 2002) of the matrix of basis functions $\boldsymbol{S}$ can be used to reduce the linear system solved in ML estimation, and also outlines how a version of the Sherman-Morrison-Woodbury formula can be utilized to reduce the linear system solved during prediction. Section 3 evaluates our methodol- 
ogy via a simulation study, and compares performance to the E-M approach. Section 4 demonstrates the applicability of our reduced-basis kriging methodology to predicting temperatures across the US. We conclude with some discussion and pointers to future work. We also have a supplement providing some additional illustrations. Sections and figures in the supplement referred to in this paper are labeled with the prefix "S-".

\section{Methodology}

\subsection{Reduced Model Reparametrization for increased computational effi- ciency}

Consider writing $\boldsymbol{S}$ in terms of its QR-decomposition (Golub and Van Loan, 1996), i.e., $\boldsymbol{S}=\boldsymbol{Q}_{1} \boldsymbol{R}_{1}$, where $\boldsymbol{R}_{1}$ is an $m \times m$ upper triangular matrix and $\boldsymbol{Q}_{1}$ is an $n \times m$ matrix being such that $\boldsymbol{Q}_{1}^{\prime} \boldsymbol{Q}_{1}=$ $\boldsymbol{I}_{m}$, the $m \times m$ identity matrix. Let $\boldsymbol{y}^{*}=\boldsymbol{Q}_{1}^{\prime} \boldsymbol{y}$, write $\boldsymbol{\epsilon}^{*}=\boldsymbol{Q}_{1}^{\prime}(\boldsymbol{\delta}+\boldsymbol{\epsilon})$, and define $\boldsymbol{D}^{*}=\boldsymbol{Q}_{1}^{\prime} \boldsymbol{D} \boldsymbol{Q}_{1}$. Then equation (3) reduces to $\boldsymbol{y}^{*}=\boldsymbol{R}_{1} \boldsymbol{\eta}+\boldsymbol{\epsilon}^{*}$, and the distribution of $\boldsymbol{y}^{*} \sim N_{m}\left(\mathbf{0}, \boldsymbol{\Sigma}^{*}\right)$ where $\boldsymbol{\Sigma}^{*}$ is defined as $\Sigma^{*}=\boldsymbol{R}_{1} \boldsymbol{K} \boldsymbol{R}_{1}^{\prime}+\boldsymbol{D}^{*}$. The likelihood function of the parameters of interest, given the transformed observations $\boldsymbol{y}^{*}$ is then given by

$$
\ell\left(\boldsymbol{K}, \sigma^{2} ; \boldsymbol{y}^{*}\right)=-\frac{1}{2} \boldsymbol{y}^{* \prime}\left(\boldsymbol{R}_{1} \boldsymbol{K} \boldsymbol{R}_{1}^{\prime}+\boldsymbol{D}^{*}\right)^{-1} \boldsymbol{y}^{*}-\frac{1}{2} \log \left(\left|\boldsymbol{R}_{1} \boldsymbol{K} \boldsymbol{R}_{1}^{\prime}+\boldsymbol{D}^{*}\right|\right)-\frac{n}{2} \log (2 \pi) .
$$

The transformation from $\boldsymbol{y}$ to $\boldsymbol{y}^{*}$ concentrates the original dataset into a smaller dataset of length $m$, located at the knots. It also reduces the size of the variance-covariance matrix of the dataset (from the original $n \times n$ matrix $\Sigma$ to the smaller $m \times m$ matrix $\Sigma^{*}$ ). A few computational benefits accrue with this reduction. For one, computations involving the determinant and the inverse of the matrices in the calculation of (5) are reduced from $O\left(n^{3}\right)$ to $O\left(m^{3}\right)$. A further speedup is obtained if we combine the matrix determinant and inverse calculations. We propose using a Cholesky decomposition (Higham, 2002) for its versatility in evaluating determinants as well as solving linear systems. The use of a Cholesky method coupled with our transformation eliminates the need to solve an additional $n \times n$ linear system to obtain the inverse of the variance-covariance matrix beyond the determinant: in its stead, we are reduced to solving a linear system involving an upper-triangular coefficient matrix, whose diagonal elements when multiplied lead to the determinant. 
A further efficiency is obtained because of the reduction of computational burden from $O\left(n^{2} m\right)$ to $O\left(\mathrm{~m}^{3}\right)$ with regard to matrix multiplication because this approach avoids the matrix multiplications involving $\Sigma^{-1}$ necessary with the E-M algorithm (4), using instead the $m \times m$ matrix $\Sigma^{*-1}$.

\subsection{Computationally Efficient Kriging Predictions}

We now turn our attention to making kriging predictions and calculating the corresponding standard errors. Using the identities $\boldsymbol{R}_{1}^{\prime} \boldsymbol{R}_{1}=\boldsymbol{S}^{\prime} \boldsymbol{S}$ and $\boldsymbol{R}_{1}^{\prime} \boldsymbol{y}^{*}=\boldsymbol{S}^{\prime} \boldsymbol{y}$, we note that the ML-estimated kriging predictor and standard errors are unchanged: $\hat{\boldsymbol{f}}\left(\boldsymbol{s}_{0}\right)=\boldsymbol{A} \hat{\boldsymbol{K}} \boldsymbol{S}^{\prime}\left(\boldsymbol{S} \hat{\boldsymbol{K}} \boldsymbol{S}^{\prime}+\hat{\boldsymbol{D}}\right)^{-1} \boldsymbol{y}$ and $\hat{\boldsymbol{\sigma}}_{k}\left(\boldsymbol{s}_{0}\right)=\left[\boldsymbol{A} \hat{\boldsymbol{K}} \boldsymbol{A}^{\prime}+\hat{\sigma}_{\delta}^{2} \boldsymbol{V}_{\delta}\left(\boldsymbol{s}_{0}\right)-\boldsymbol{A} \hat{\boldsymbol{K}} \boldsymbol{S}^{\prime}\left(\boldsymbol{S} \hat{\boldsymbol{K}} \boldsymbol{S}^{\prime}+\hat{\boldsymbol{D}}\right)^{-1} \boldsymbol{S} \hat{\boldsymbol{K}} \boldsymbol{A}^{\prime}\right]^{\frac{1}{2}}$. Note that both equations need the product $\hat{\boldsymbol{K}} \boldsymbol{S}^{\prime}\left(\boldsymbol{S} \hat{\boldsymbol{K}} \boldsymbol{S}^{\prime}+\hat{\boldsymbol{D}}\right)^{-1}$. To simplify calculations, we use the following matrix identity provided in Searle (2006) or in Peterson and Pedersen (2008): Let $\boldsymbol{B}$ be a $n \times n$ invertible matrix and let $\boldsymbol{C}$ be any $n \times m$ matrix such that $\boldsymbol{B}+\boldsymbol{C} \boldsymbol{C}^{\prime}$ is invertible. Then $\boldsymbol{C}^{\prime}\left(\boldsymbol{B}+\boldsymbol{C} \boldsymbol{C}^{\prime}\right)^{-1}=$ $\left(\boldsymbol{I}+\boldsymbol{C}^{\prime} \boldsymbol{B}^{-1} \boldsymbol{C}\right)^{-1} \boldsymbol{C}^{\prime} \boldsymbol{B}^{-1}$. This result can then be used to obtain a special case of the Woodbury formula, known for its use in Kalman filtering as an alternative algorithm (Peterson and Pedersen, 2008; Brown and Hwang, 1992). Let $\boldsymbol{S}$ be a $n \times m$ matrix ( $m<n$, of rank $m$ ). Further, let $\boldsymbol{K}$ and $\boldsymbol{D}$ be positive definite matrices of real values of rank $m$ and $n$, respectively, with unique square root matrix $\boldsymbol{K}^{\frac{1}{2}}$. Then $\boldsymbol{K} \boldsymbol{S}^{\prime}\left(\boldsymbol{S} \boldsymbol{K} \boldsymbol{S}^{\prime}+\boldsymbol{D}\right)^{-1}=\left(\boldsymbol{K}^{-1}+\boldsymbol{S}^{\prime} \boldsymbol{D}^{-1} \boldsymbol{S}\right)^{-1} \boldsymbol{S}^{\prime} \boldsymbol{D}^{-1}$. (For a full proof, refer to the supplementary material.) This means that the kriging predictor reduces to

$$
\hat{\boldsymbol{f}}\left(\boldsymbol{s}_{0}\right)=\boldsymbol{A}\left(\boldsymbol{S}^{\prime} \hat{\boldsymbol{D}}^{-1} \boldsymbol{S}+\hat{\boldsymbol{K}}^{-1}\right)^{-1} \boldsymbol{S}^{\prime} \hat{\boldsymbol{D}}^{-1} \boldsymbol{y}
$$

while the kriging standard error becomes

$$
\begin{aligned}
\hat{\boldsymbol{\sigma}}_{k}\left(\boldsymbol{s}_{0}\right)=\left[\boldsymbol{A} \hat{\boldsymbol{K}} \boldsymbol{A}^{\prime}+\hat{\sigma}_{\delta}^{2} \boldsymbol{V}_{\delta}\left(\boldsymbol{s}_{0}\right)-\right. & \\
& \left.\boldsymbol{A}\left(\boldsymbol{S}^{\prime} \hat{\boldsymbol{D}}^{-1} \boldsymbol{S}+\hat{\boldsymbol{K}}^{-1}\right)^{-1} \boldsymbol{S}^{\prime} \hat{\boldsymbol{D}}^{-1} \boldsymbol{S} \hat{\boldsymbol{K}} \boldsymbol{A}^{\prime}\right]^{\frac{1}{2}} .
\end{aligned}
$$

thus bringing down the computational effort needed in the matrix multiplication in the kriging predictor. Specifically, we note that in (6) and (7), we invert two $m \times m$ matrices, namely $\hat{\boldsymbol{K}}$ and $\boldsymbol{S}^{\prime} \boldsymbol{D}^{-1} \boldsymbol{S}+\hat{\boldsymbol{K}}^{-1}$, with each an $O\left(m^{3}\right)$ operation - instead of the $O\left(n^{3}\right)$ operation of inverting one $n \times n$ matrix. Both (6) and (7) also require significantly fewer matrix operations: thus for large $n$ 
(and especially when $m<<n$ ), there are substantial gains in computational efficiency in using these approaches.

The material developed in Sections 2.1 and Section 2.2 have presented the wherewithal to further reduce the $\mathrm{CPU}$ time and memory required to perform fixed-rank kriging. Because our simplifications are built on a setup with a reduced set of basis functions, we call our developed methodology "Reduced Basis Kriging". In general, while reduced basis kriging has the ability to bring down computational overhead, it should be noted that the exact form of the basis functions and the covariance structure also play a significant role on computational efficiency. We next discuss specific implementations of reduced basis kriging that address these issues.

\subsection{Issues in Implementation for Computational Efficiency}

\subsubsection{Choice of Basis Functions}

In general, the specific choice of basis functions is subjective since it is difficult to estimate from data, however it is common to use a multi-resolution smoothing function (Cressie and Johannesson, 2008) which defines the lth resolution basis function as

$$
S_{k(l)}(\boldsymbol{x})=\Psi\left(\frac{\left\|\boldsymbol{x}-\boldsymbol{u}_{k(l)}\right\|}{r_{l}}\right) \quad \forall \boldsymbol{x} \in D,
$$

where $\Psi(d)$ is the smoothing function. A commonly used $\Psi(d)$ is the local bisquare function defined as

$$
\Psi(d)= \begin{cases}\left(1-d^{2}\right)^{2} & 0 \leq d \leq 1 \\ 0 & d>1\end{cases}
$$

and $r_{l}=b \min \left\{\left\|\boldsymbol{u}_{i(l)}-\boldsymbol{u}_{j(l)}\right\|: j \neq i, 1 \leq i, j \leq m\right\}$. We follow Cressie and Johannesson (2008) and use the multi-resolution local bisquare function (8) together with (9) in our illustrations. In our paper, we have assumed that all distances are defined in terms of the Euclidean norm. Further, $r_{l}$ is a standardizing quantity that is known as the "bandwidth" of the basis function where $b$ is some constant.

The local bisquare function (9) sets any value equal to zero where the distance between the location and the knot is greater than $r_{l}$. This means that the matrices $\boldsymbol{S}$ and $\boldsymbol{A}$ are sparse as long as the $r_{l}$ for each resolution is not too large. Recognition of this aspect of $\boldsymbol{S}$ and $\boldsymbol{A}$ presents some computational advantages because we can now utilize matrix operations and algorithms (Davis, 
2006) that have been specifically designed to exploit sparsity and can therefore handle larger matrices. Such algorithms are computationally advantageous because they provide memory savings and allow for fast matrix manipulations. This is because they can be compressed into memoryreduced forms because only non-zero values and their corresponding indexes require storage. There are savings in computer processing time also because manipulations involving sparse matrices utilize the sparsity to reduce the number of calculations performed. In this paper, we used the R package Matrix (Bates and Maechler, 2015) which uses functions from the LAPACK (Anderson et al., 1999) and SuiteSparse (Davis, 2006) packages for fast matrix manipulations such as in efficient computation of QR-decomposition algorithms.

Much of the computational advantage of using a sparse matrix lies in the degree of sparsity that exists. Given that a local bisquare function is used for the basis functions, the bandwidth constant $(b)$ is a crucial factor in determining the amount of computational reduction we can expect. An exact estimate of $b$ may not be necessary, but a suitable range for $b$ should be determined to ensure fast computations and precise predictions. In their work, Cressie and Johannesson (2008) suggested a bandwidth constant of 1.5. In this paper, we investigate the effects of a range of values of $b$ through a series of simulation experiments.

\subsubsection{Choice of $K$}

The other a priori specification that can greatly affect prediction is the form of $\boldsymbol{K}$ which defines the spatial covariance between knots. The Spatial Random Effects model (Cressie and Johannesson, 2008) does not restrict $\boldsymbol{K}$ to any particular form, however it is important to understand what effect a smoothing function, in particular the local bisquare function, will have on the covariance of the reduced spatial process. We discuss the effect of $\boldsymbol{K}$ by first specifying the covariance structure for the original spatial process.

There are several choices for the spatial covariance structure (Cressie, 1993). For this particular paper, we will assume that the original spatial covariance, $\Sigma_{f}$, can be modeled in terms of a covariance matrix from the Matérn family (Stein, 1999), which is a common choice for spatial data. The Matérn covariance between $\boldsymbol{f}\left(\boldsymbol{s}_{i}\right)$ and $\boldsymbol{f}\left(\boldsymbol{s}_{j}\right)$ is defined by $\operatorname{Cov}\left[\boldsymbol{f}\left(\boldsymbol{s}_{i}\right), \boldsymbol{f}\left(\boldsymbol{s}_{j}\right)\right]=$ $\frac{\rho}{2^{\nu-1} \Gamma(\nu)}\left(\frac{\left\|s_{i}-s_{j}\right\|}{\theta}\right)^{\nu} K_{\nu}\left(\frac{\left\|s_{i}-s_{j}\right\|}{\theta}\right)$, where $K_{\nu}$ is the modified Bessel function of the second kind of order $\nu, \nu$ is the smoothness parameter, $\rho$ is the sill parameter, and $\theta$ is the range parameter, with 
$\nu, \theta \in(0, \infty)$ and $\rho \in[0, \infty)$. Note that given the assumption of the Euclidean norm, the Matérn covariance function is second-order stationary in our setup.

It is paramount to understand how the fixed rank kriging approximations model common forms of spatial variance. Thus, the form of $\boldsymbol{K}$ when $\boldsymbol{\Sigma}_{f}$ is Matérn is of significant importance. Although the exact form of $\boldsymbol{K}$ is not easily recovered, an empirical covariance structure can be obtained by combining the QR-decomposition of $\boldsymbol{S}$ with an eigenvalue decomposition of $\boldsymbol{\Sigma}$ (Higham, 2002). Specifically, writing $\Sigma_{f}$ in terms of its spectral decomposition $\Sigma_{f}=\boldsymbol{P} \boldsymbol{\Lambda} \boldsymbol{P}^{\prime}$. Write $\boldsymbol{P}=\left[\boldsymbol{P}_{1}: \boldsymbol{P}_{2}\right]$, where $\boldsymbol{P}_{1}$ contains the first $m$ columns of $\boldsymbol{P}$, and let $\boldsymbol{\Lambda}_{1}$ be the diagonal matrix containing the $m$ eigenvalues corresponding to the eigenvectors in $\boldsymbol{P}_{1}$. Then, since $\boldsymbol{f}(\boldsymbol{s})=\sum_{k=1}^{m} \boldsymbol{S}_{k}(\boldsymbol{s}) \boldsymbol{a}\left(\boldsymbol{u}_{k}\right)$, we have that $\boldsymbol{\Sigma}_{f}=\boldsymbol{S} \boldsymbol{K} \boldsymbol{S}^{\prime}$ and

$$
\boldsymbol{K}=\boldsymbol{R}_{1}^{-1} \boldsymbol{Q}_{1}^{\prime} \boldsymbol{P}_{1} \boldsymbol{\Lambda}_{1} \boldsymbol{P}_{1}^{\prime} \boldsymbol{Q}_{1}\left(\boldsymbol{R}_{1}^{-1}\right)^{\prime}
$$

Katzfuss and Cressie (2011) noted how the usual form of maximum likelihood estimation of $\boldsymbol{K}$ was problematic because it required maintaining a positive definite matrix through a numerical optimizing technique. Thus, in addition to identifying a form of $\boldsymbol{K}$ that will be computationally efficient and produce accurate predictions, a numerically stable form is also important. In the next section, we investigate the behavior of $\boldsymbol{K}$ to conclude a reasonable covariance structure. We will also explore, through simulation, the loss in accuracy that may be caused by using an approximate but simpler model $(\boldsymbol{K} \propto \boldsymbol{I})$, and outline methods for minimizing this loss by properly constructing the basis functions.

We conclude this section briefly reiterating that our setup here (and in our experiments) has assumed second order stationarity. In cases when the assumption can not be sustained, tactics such as detrending and median-polishing (Hoaglin et al., 1983) may be adopted to transform the observations into a second order stationary process.

\section{Experimental Evaluations}

\subsection{Simulation Details}

We investigated the behavior of our reduced basis kriging methodology through a series of simulation experiments using the local bisquare basis function of (8) and a Matérn covariance struc- 
ture for the original spatial process. For simplicity, we assume a Gaussian random spatial field with second order stationarity and no measurement error, $\sigma_{\epsilon}^{2}=0$.

We investigated the effect of fine-scale variation in our simulation experiments by setting $\sigma_{\delta}^{2}$ to be equal to $0,0.1,0.25$, and 0.4 , while holding $\rho$ constant at unity and set $\boldsymbol{V}_{\delta}=\boldsymbol{I}$ for simplicity. The smoothness parameter $\nu$, is most commonly set to be between 0.5 and 2 , so we let $\nu$ be over the set of values in $\{0.5,1,1.5,2\}$. Note that the range parameter, $\theta$, is dependent on $\nu$. Therefore the value of $\theta$ was paired to $\nu$ so that the correlation would be 0.2 between observations at a distance of $1 / 3$ of the spatial domain. Specifically, $\theta$ was set to be equal to $0.205,0.137,0.110$, and 0.095 , respectively.

The domain for the simulation experiments consisted of two grids $(50 \times 50$ or $200 \times 200)$ with locations ranging from 0 to 1 . Three hundred randomly selected locations out of the possible 2500 or 40000 represented the locations of the observations with varying degrees of missing data. The selection of basis functions depends on a variety of factors: these are the number of knots $(m)$, the location of the knots, the number of resolutions $(l)$, and the bandwidth constant (b). These variables all need specification prior to implementation of fixed rank kriging. Since the simulated spatial field is relatively small and without much complexity, using one resolution always produced predicted values with the lowest corresponding mean square prediction error. Thus, the focus of the results in this paper will be on estimation and prediction using a single resolution, $l=1$. The values for $m$ and $b$, in contrast, are presented varied to test the effect on time and accuracy.

A regular, triangular grid was chosen for the knot location after preliminary results suggested that different space-filling designs for the locations of the knots had an insignificant effect on prediction when the observations were not clustered. The number of knots used were $m=23$, $m=77$ or $m=175$, which represented a reduction of roughly $5 \%$ to $50 \%$ of $n$. Cressie and Johannesson (2008) suggested a bandwidth of 1.5 multiplied by the shortest distance between knots. Thus, in our experiments, we tested our method for bandwidths ranging from 0.5 to 2.5 in increments of 0.1 and multiplied by the shortest distance between knots. 


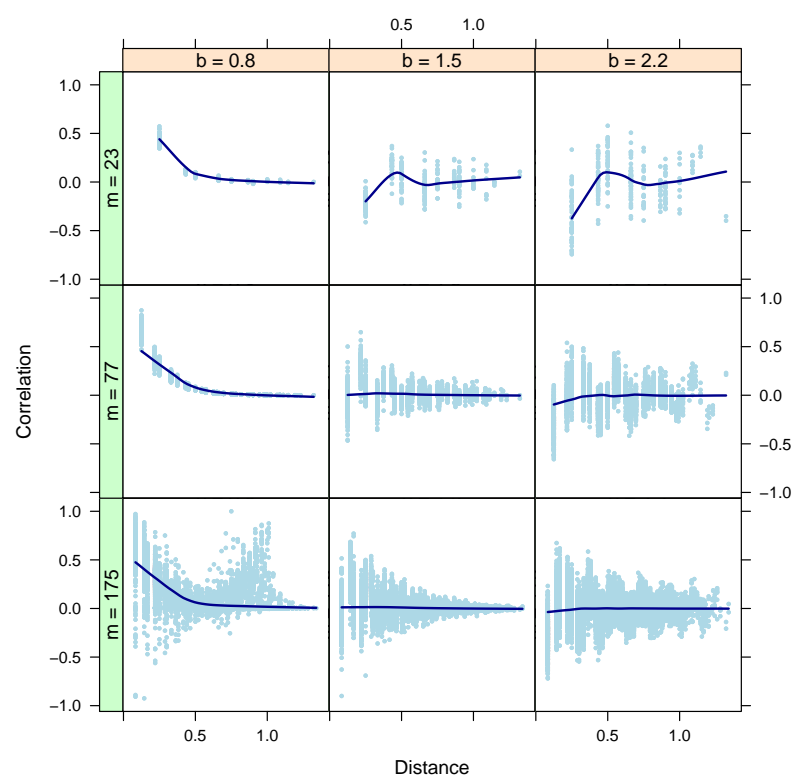

Figure 1: Correlation between knots from a $50 \times 50$ grid plotted against distance for $\nu=1$, $\theta=0.137, \sigma^{2}=0.25$.

\subsection{Covariance of the Reduced Spatial Process}

Using the setup from the previous subsection and the method outlined in (10), we simulated $\boldsymbol{K}$ for every combination of parameter values, number of knots, and bandwidth constants and converted the covariance into a correlation. Figure 1 provides an example of that correlation relative to distance (excluding the superfluous case where distance $=0$ ) for all $m$ at select bandwidth constants on a $50 \times 50$ grid when $\nu=1, \theta=0.137, \sigma^{2}=0.25$. (All other combinations of grid size, bandwidth constant, and parameter values yielded figures of the same kind and are omitted for brevity.)

The stationarity commonly visible in a Matérn covariance is clearly weakened by the use of basis functions to describe the large scale spatial structure. Some of the Matérn structure is maintained when using lower bandwidth constants, particularly for lower $m$ and a wave pattern is often visible for bandwidth constants larger than one. The majority of the plots, however, suggest that covariance structures such as a Matérn may be somewhat needlessly and overly complex for describing the correlation between knots at varying distances and could be simplified to a matrix proportional to the identity matrix. Choosing $\boldsymbol{K}$ to be proportional to the identity matrix 
is particularly attractive because its simplicity can provide huge computational gains while also increasing the stability in the numerical optimization steps.

\subsection{Simulation Results}

One hundred Gaussian random fields were simulated using the R package fields (Nychka et al., 2015b) with a single resolution for each set of parameter values. For each field, fine-scale errors were added to each response at the 300 randomly selected locations, resulting in values that represented the dataset. Then, we acquired ML estimates and the corresponding predicted values for each simulated field using three different methods: by optimizing (5) using an identity covariance for $\boldsymbol{K}=\rho \boldsymbol{I}$ and utilizing (6), by the E-M approach (4) using the full form of $\boldsymbol{K}$, and by the E-M approach approximating $\boldsymbol{K}$ with the identity matrix, $\rho \boldsymbol{I}$. As mentioned in Section 2, these computations were done using sparse matrix methods (Davis, 2006) implemented as per the R package "Matrix" (Bates and Maechler, 2015).

The results of our experiments indicated that the simplicity of the identity covariance for $\boldsymbol{K}$ resulted in an expected gain in computational efficiency. To illustrate this gain, the time required to iterate to convergence and obtain predicted values for each combination of inputs was recorded. This value combines the cost of inverting a matrix with a penalty for covariance structures that require additional iterations to meet convergence. Example plots of the distributions of seconds on the log scale for one resolution and varying number of knots, bandwidth constants, and estimation methods on a $50 \times 50$ grid when $\nu=1$ and $\sigma_{\delta}^{2}=.25$ are provided in Figure 2 (left panel). These boxplots display all three estimation methods: reduced basis kriging (blue), the E-M with an identity covariance for $\boldsymbol{K}$ (gold), and the E-M with a full covariance for $\boldsymbol{K}$ for fixed $b=1.5$ (white). The horizontal line represents the median seconds for the E-M approach using a full $\boldsymbol{K}$. For clarity of presentation, the distribution of log seconds for the E-M approach using the full form of $\boldsymbol{K}$ are presented separately when the maximum is significantly beyond the range of the corresponding results for the identity form of $\boldsymbol{K}$, such as in Figure 2(c). These example plots represent the patterns seen throughout all input combinations. Additional figures (see Figures S-1 through S-16) in the supplementary material also support the following conclusions.

Fewer knots resulted in less computation time for the E-M approach and a bandwidth con- 
stant near unity provided the optimal level of sparsity in $S$ in terms of CPU time. An interesting difference between the E-M approach and reduced basis kriging is the robustness of reduced basis kriging to increasing $m$ or a poor choice of bandwidth constant. Reduced basis kriging is efficient regardless of the specifics of the basis functions used and is at least as efficient as the $\mathrm{E}-\mathrm{M}$ approach with the identity covariance for any choice of $b$. The E-M approach is consistently more computationally expensive for larger values of $b$.

Although reduced basis kriging improves computational efficiency, the additional compression of the data could increase prediction error. To assess accuracy the mean square prediction error (MSPE) was computed relative the true simulated field. Figure 2 (right panel) also provides the distributions of MSPE on a $50 \times 50$ grid when $\nu=1$ and $\sigma_{\delta}^{2}=.25$. These boxplots are organized as before, with the horizontal line representing the median MSPE for the E-M approach using a full $\boldsymbol{K}$. Once again, these example plots represent the patterns seen throughout all input combinations.

The distributions of MSPE show that for a reasonable $b$, the identity covariance structure is at least as accurate in terms of prediction as the full covariance structure. In fact, as $m$ approaches $n$, the identity covariance performs substantially better than the full covariance with regards to both median and maximum MSPE. This suggests that added complexity to $\boldsymbol{K}$ is unnecessary when our focus is on prediction. Considering its simplicity and the speed of estimation, proceeding sections will focus only on spatial prediction using the identity covariance model.

Using the identity covariance for $\boldsymbol{K}$, reduced basis kriging and the E-M approach are comparable for choices of bandwidth constant where MSPE is minimized, however an unreasonable $b$ favors the E-M approach in terms of MSPE. This is opposite of computational efficiency, in which reduced basis kriging was more robust to poor choices of $b$. Fortunately, the substantial reduction in computation achieved by using the identity covariance coupled with parallel computing allow for the estimation of multiple models (varying $b$ and the number of resolutions). A model selection approach can thus be implemented by minimizing MSPE through cross-validation. However, as a continuous variable, the domain of $b$ is infinite and thus a model selection approach is less than ideal. Selection of an appropriate value for $b$ and the correct number of resolutions are paramount to producing accurate predictions and will be explored in further work.

Provided a reasonable bandwidth and number of resolutions can be obtained, reduced basis 


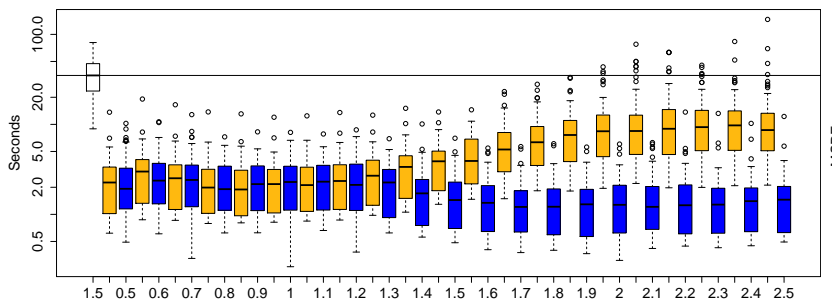

(a) $m=23$

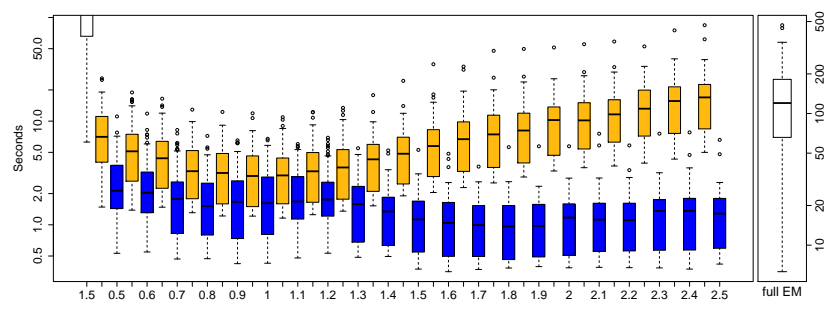

(c) $m=77$

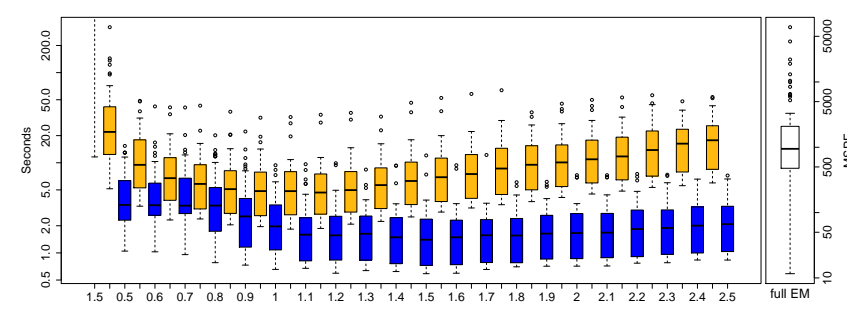

(e) $m=175$

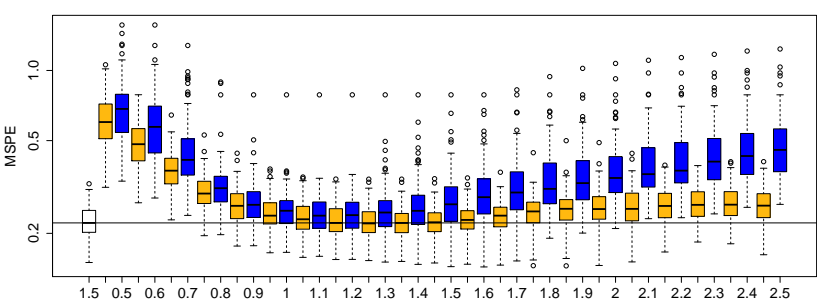

(b) $m=23$

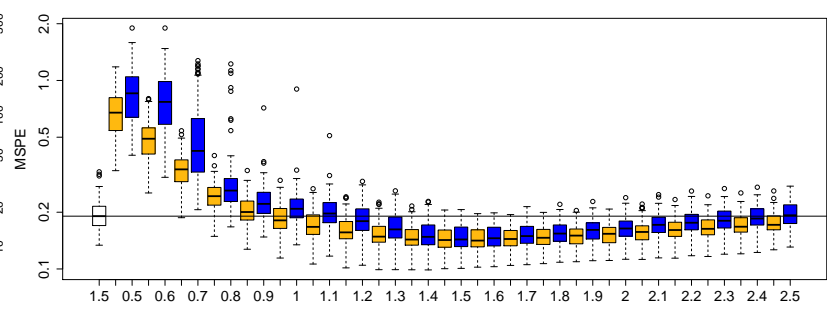

(d) $m=77$

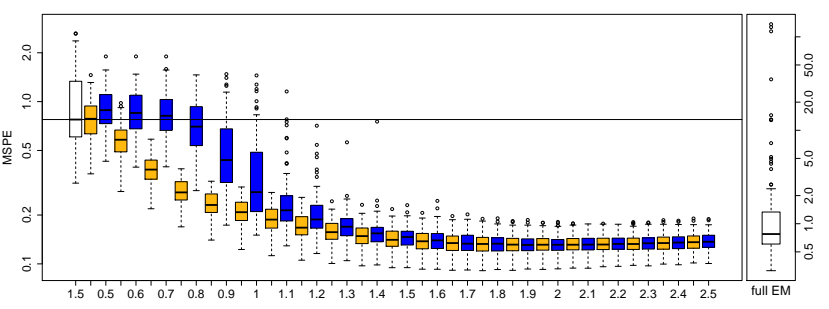

(f) $m=175$

Figure 2: Seconds (left panel) and Mean Squared Prediction Errors (MSPEs, right panel) for varying bandwidths constants (x-axis) and number of knots $(m)$ for all three estimation methods on a $50 \times 50$ grid when $\nu=1$ and $\sigma^{2}=.25$. The white boxplot is the E-M method with a full covariance for $K$ for fixed $b=1.5$. 
kriging and the E-M approach using the identity covariance produce very comparable distributions of MSPE. The major advantage to reduced basis kriging is that the memory necessary to perform each iteration of the estimation process is greatly reduced by concentrating the data from length $n$ to length $m$. Distributions of seconds for bandwidth constants that minimize MSPE ( $b \approx 1.3$ for $m=23, b \approx 1.5$ for $m=77$, and $b \approx 2$ for $m=175$ ) illustrate a distinct advantage to using reduced basis kriging. All patterns visible in Figure 2 were also consistent for all other combinations of parameter and grid values, which can be found in the supplementary materials.

In summary, the results of our experiments show that the identity form of $\boldsymbol{K}$ is optimal in terms of minimizing computational cost without sacrificing accurate prediction, provided that a reasonable bandwidth constant and resolution are selected. The massive computational gains achieved by simplifying the covariance between knots allows for the estimation of numerous "models", leading us to a model selection approach that is achieved by minimizing the mean kriging standard error, $\hat{\boldsymbol{\sigma}}_{k}\left(\boldsymbol{s}_{0}\right)$. Additional and robust computational gains are also achieved by reduced basis kriging with a minimal increase in MSPE. We now apply our methodology to the National Climate Data Center (NCDC) data of monthly temperatures recorded across the continental United States of America.

\section{Predicting temperatures over the continental US}

The Cooperative Observer Program (COOP), formally established in 1890, is the nation's largest and oldest weather and climate observing network (National Weather Service, 2000), consisting of over 11,700 volunteer citizens and institutions observing and reporting weather information on a 24-hour basis. The data is archived at the US National Climate Data Center (NCDC) and available online at http://www.image.ucar.edu/Data/US.monthly.met/.

A basic summary in climate science is provided by mean temperature and precipitation fields on a regular grid (Johns et al., 2003). One important application is to compare these fields from observational data to those simulated by climate derived models. For our example, we consider mean temperatures in April in 1990 observed over the entire contiguous United States of America. Thus kriging the observed field is important in this setting.

For our example, we use mean temperatures in April in 1990 observed over the entire con- 
tinental United States. The daily minimum and maximum temperatures were observed at 5030 locations across the United States and the mean monthly minimum and maximum temperatures were calculated. To obtain an overall monthly average, the mean monthly minimum and maximum temperatures were averaged together. This average will be our "monthly mean" temperature.

Under the normal kriging method, prediction using the entire U.S. observational record would require a Cholesky decomposition on a matrix of dimension $5030 \times 5030$, which can be computationally prohibitive, both in terms of CPU time and memory. Additionally, obtaining kriging predictions generally requires the use of an iterative process to estimate the parameter values of the model, meaning that this expensive calculation will need to be repeated. Thus, the normal kriging approach clearly becomes impractical for large data sets, and we are led to approaches such as reduced basis kriging.

\subsection{Data Preprocessing}

Intrinsic stationarity is not a reasonable assumption for this data. Temperature is directly affected by latitude, longitude, and elevation and so the spatial field does not have a constant mean. A simple approach to adjust for the non-stationarity due to these factors is to use the additive model $\boldsymbol{y}(\boldsymbol{s}, \boldsymbol{h}, \boldsymbol{u})=\boldsymbol{g}_{\text {Elev }}(\boldsymbol{h}(\boldsymbol{s}))+\boldsymbol{g}_{\text {Lat }}(\boldsymbol{s})+\boldsymbol{g}_{\text {Lon }}(\boldsymbol{s})+\boldsymbol{S}(\boldsymbol{s}, \boldsymbol{u}) \boldsymbol{\eta}(\boldsymbol{u})+\boldsymbol{\delta}(\boldsymbol{s})+\boldsymbol{\epsilon}(\boldsymbol{s})$ where $\boldsymbol{h}(\boldsymbol{s})$ is the elevation at location $\boldsymbol{s}, \boldsymbol{u}$ are the knots, $\boldsymbol{\eta}(\boldsymbol{u}) \sim N(\mathbf{0}, \rho \boldsymbol{I}), \boldsymbol{\delta}(\boldsymbol{s}) \sim N\left(\mathbf{0}, \sigma_{\delta}^{2} \boldsymbol{I}\right)$ and $\boldsymbol{\epsilon}(\boldsymbol{s}) \sim N\left(\mathbf{0}, \sigma_{\epsilon}^{2} \boldsymbol{I}\right)$, and $\boldsymbol{\eta}(\boldsymbol{u}), \boldsymbol{\delta}(\boldsymbol{s})$, and $\boldsymbol{\epsilon}(\boldsymbol{s})$ are mutually independent.

We estimated $\boldsymbol{g}_{\text {Elev }}(\boldsymbol{h}(\boldsymbol{s}))$ by a cubic regression spline with five degrees of freedom to the data, $\boldsymbol{g}_{\text {Lat }}(\boldsymbol{s})$ in terms of a quadratic regression spline with 4 degrees of freedom, and $\boldsymbol{g}_{\text {Lon }}(\boldsymbol{s})$ using a cubic regression spline with 6 degrees of freedom, all fit to the dataset by AIC. These splines are shown in Figure 3 and demonstrate that the majority of the mean structure is captured.

\subsection{Results and Analysis}

We begin with the linear equation, $\boldsymbol{y}=\boldsymbol{X} \boldsymbol{\alpha}+\boldsymbol{S} \boldsymbol{\eta}+\boldsymbol{\delta}+\boldsymbol{\epsilon}$ and define the $\mathrm{QR}$-decompositions $\boldsymbol{X}=\left[\boldsymbol{Q}_{\boldsymbol{X} 1}, \boldsymbol{Q}_{\boldsymbol{X}_{2}}\right]\left[\boldsymbol{R}_{\boldsymbol{X}}, \mathbf{0}\right]^{\prime}$ and $\tilde{\boldsymbol{S}}=\boldsymbol{Q}_{1} \boldsymbol{R}_{1}$. Given this additive model, a QR-decomposition of the basis matrix for the polynomial splines was used to remove the trend in the data by multi- 


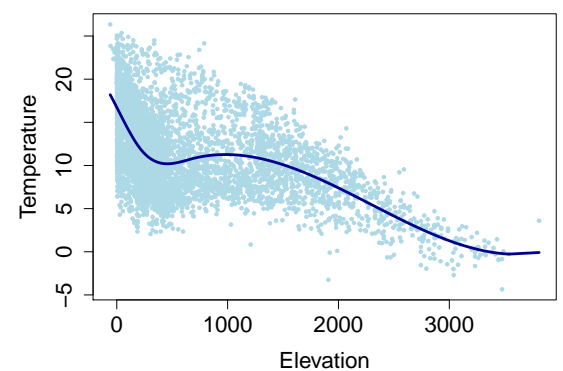

(a) Cubic regression spline

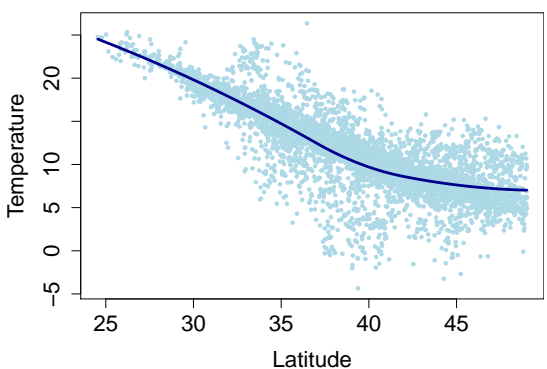

(b) Quadratic regression spline

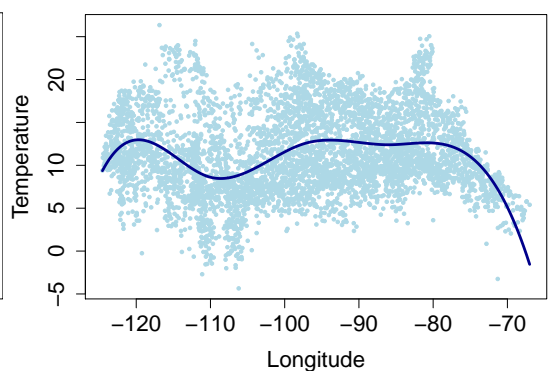

(c) Cubic regression spline

Figure 3: Monthly mean temperature plotted against covariates with regression spline.

plying through by the portion of $\boldsymbol{Q}_{\boldsymbol{X}}$ that is orthogonal to $\boldsymbol{X}$, as shown in equation (11). Thus, the effects of location and elevation are removed from the observations. Reduced basis kriging was subsequently performed on the detrended data set, $\tilde{\boldsymbol{y}}$, and least-squares estimates of the regression splines were used to add the effects of elevation and latitude back into the response following reduced basis kriging.

$$
\begin{aligned}
y & =\boldsymbol{X} \boldsymbol{\alpha}+\boldsymbol{S} \boldsymbol{\eta}+\boldsymbol{\delta}+\boldsymbol{\epsilon} \\
\boldsymbol{X} & =\left[\begin{array}{ll}
Q_{X 1} & Q_{X 2}
\end{array}\right]\left[\begin{array}{c}
\boldsymbol{R}_{X} \\
0
\end{array}\right]
\end{aligned}
$$

Then,

$$
\begin{aligned}
\boldsymbol{Q}_{\boldsymbol{X} 2}^{\prime} \boldsymbol{y} & =\boldsymbol{Q}_{\boldsymbol{X} 2}^{\prime} \boldsymbol{X} \boldsymbol{\alpha}+\boldsymbol{Q}_{\boldsymbol{X} 2}^{\prime} \boldsymbol{S} \boldsymbol{\eta}+\boldsymbol{Q}_{\boldsymbol{X}_{2}}^{\prime}(\boldsymbol{\delta}+\boldsymbol{\epsilon}) \\
\Rightarrow \tilde{\boldsymbol{y}} & =\tilde{\boldsymbol{S}} \boldsymbol{\eta}+\tilde{\boldsymbol{\epsilon}}, \quad \text { where } \tilde{\boldsymbol{S}}=\boldsymbol{Q}_{1} \boldsymbol{R}_{1}, \\
\text { and } \boldsymbol{Q}_{1}^{\prime} \tilde{\boldsymbol{y}} & =\boldsymbol{Q}_{1}^{\prime} \tilde{\boldsymbol{S}} \boldsymbol{\eta}+\boldsymbol{Q}_{1}^{\prime} \tilde{\boldsymbol{\epsilon}} \Rightarrow \boldsymbol{y}^{*}=\boldsymbol{R}_{1} \boldsymbol{\eta}+\boldsymbol{\epsilon}^{*}
\end{aligned}
$$

The knots $(m=501)$ were selected on a regular triangular grid within the U.S. and tested on three levels of resolution. Basis functions with a single resolution and a bandwidth constant of $b=1.6$ fit the best based on minimizing the mean kriging standard error. When run on a standard laptop with 6MB of RAM and a 64-bit Intel dual-core i5 processor, estimation was complete in 4.17 seconds and the entire process of cleaning the data, defining the matrix of basis func- 


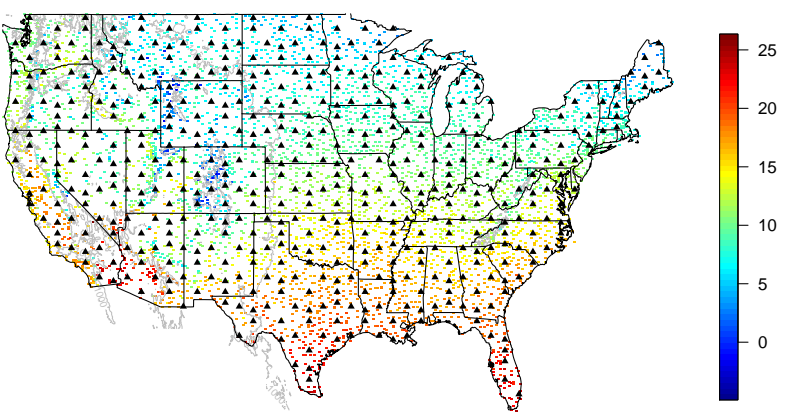

(a) Observations

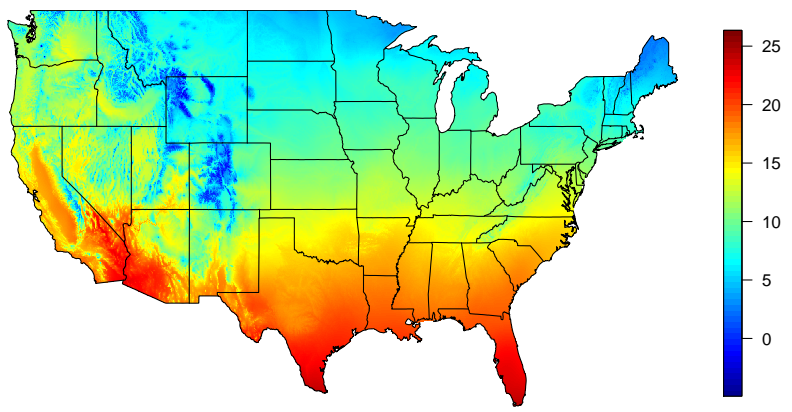

(c) Kriging estimates plus covariates

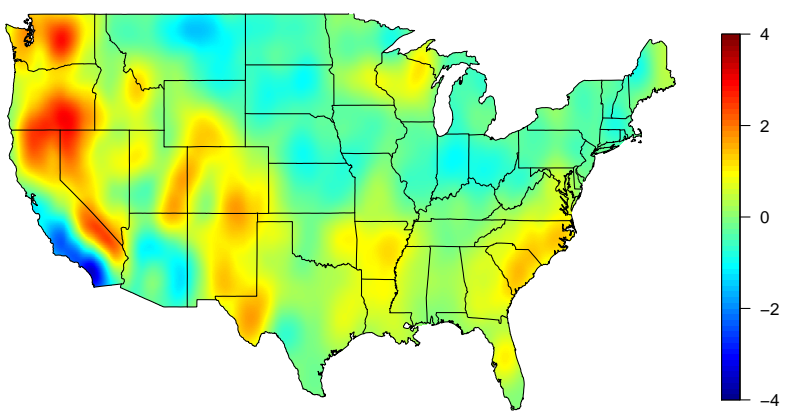

(b) Kriging estimates only

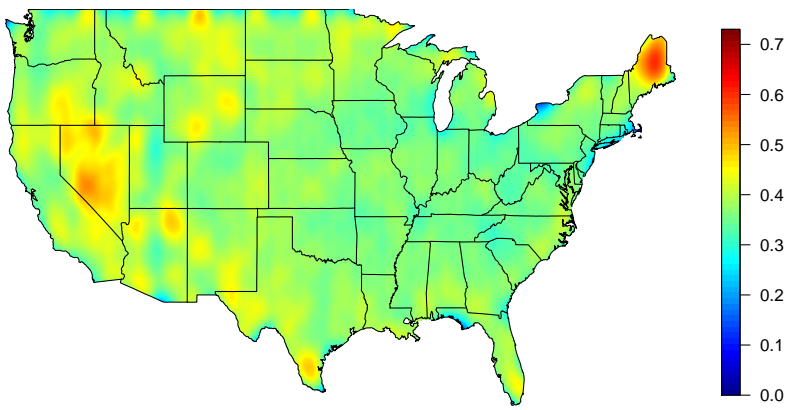

(d) Kriging standard errors

Figure 4: Mean temperatures in the US in April 1990; (a) displays the 5030 observations using 501 knots (black triangles) at one resolution, (b) depicts the reduced basis kriging estimates only, and (c) shows the sum of the spline regression predicted values for elevation, latitude, and longitude with the kriging estimates. (d) shows the kriging standard errors.

tions, detrending, estimation, prediction, and plotting results took around 3 minutes. Figure 4 summarizes the results.

From the figure, it is easy to see that elevation, latitude, and longitude are clearly shown to be useful predictors. The kriging estimates capture the additional warmth of states northwest of Texas, despite their high altitude, and the temperature difference between western and eastern sides of the Appalachian Mountains. In addition, the mild climate of the Pacific coast line is by achieved by negative kriging values in southern California and positive values in northern California, Oregon, and Washington. The kriging standard errors show relatively uniform variability, with higher variability in areas where weather stations are scarce, such as Nevada and Maine. There is also less variability in New York along the Erie and Ontario lake coast lines and in other 
areas that are along borders of the US. The systematic pattern visible in the kriging errors can be attributed to using basis functions on a regular grid. In particular, the alternating pattern visible along the US-Canada border is a result of the placement of regularly spaced knots near the border of the domain.

\section{Discussion}

This paper presents a method for performing spatial prediction efficiently and effectively when data sets are large. This is accomplished by first approximating the original spatial process using the spatial random effects model which separates the spatial covariance into a linear combination of basis functions and a reduced rank spatial process. With the approximate spatial process, a $\mathrm{QR}$-decomposition is used to reduce the dimensionality of the covariance matrix that is then solved for maximum likelihood estimation. Finally, an alternative Sherman-Morrison-Woodbury formula is invoked to obtain the predicted values over a spatial grid with increased efficiency.

Using reduced basis kriging as opposed to the E-M approach requires less memory and, thus, is beneficial when implementing kriging on large data sets. Also, in using basis functions to interpolate from the original observations to the knots, much of the spatial dependence between locations becomes absorbed in the basis functions when the bandwidth is relative large. In these situations and when the primary goal is prediction, a simplistic covariance between knots $(K)$ can be sufficient to achieve high accuracy. Consequently, it is essential that the basis functions are properly constructed, that is to say, the bandwidth and number of resolutions are carefully selected. Given the substantial computational gains achieved by a simplified covariance structure and the ability to utilize parallel processing, optimal bandwidth and number of resolutions can be obtained through model selection characterized by minimizing mean square prediction error through cross-validation. Investigation into the proper form of the basis functions is ongoing.

Although reduced basis kriging should be applicable to any Gaussian random field with intrinsic stationarity, the previous conclusions pertain to when the basis functions are local bisquare functions and only applies to situations where a Matérn covariance structure could be assumed

on the original spatial process. Reduced basis kriging provides an algorithm for simplifying the process down to the aspects essential for accurate interpolation while maintaining the advan- 
tage of quantifying uncertainty gained through kriging. However, the added flexibility of the E-M approach is preferred when estimation of the covariance between knots is of importance. Often, when data is collected over space, it is also defined by a temporal component. This work focuses on predictions for spatial data at a fixed point in time, so a spatio-temporal model would be required for more complex data.

There are several topics for future research. As mentioned, the bandwidth constant and the number of resolutions is currently being investigated. Optimal knot placement is another aspect of basis function identification that is of interest. Simulations were attempted using both a regular grid and a stratified sampling technique, without an obvious difference in performance, but these results apply only to randomly located observations and thus a more rigorous analysis would be required to come to any definite conclusions. Alternative basis functions could possibly be used to adjust for directional dependence. Recent work by Katzfuss (2016) which allows for flexible basis functions shows promise in this area. Also, considering that the accuracy of these methods were quantified by means of mean square prediction error, another area for future work would be in investigating the effects of this criteria on kriging predictions errors. Thus, we see that while we have made contributions, there is additional work to be done.

\section{Acknowledgment}

This research was supported, in part, by National Science Foundation (NSF) grant DMS-0707069, and by NSF grant DMS-CAREER-0437555. The National Center for Atmospheric Research is managed by the University Corporation for Atmospheric Research under the sponsorship of the NSF. An earlier version of this manuscript won Karl Pazdernik the 2012 Joint Statistical Meetings (JSM) Student Paper competition award sponsored by the American Statistical Association (ASA) Sections on Statistical Computing and Statistical Graphics.

\section{Supplementary Materials}

Additional Investigations: Figures S-1 through S-16 are in the supplementary materials archive of the journal website. 
R-code: R-code used to perform the estimation and prediction methods described in the article is included.

\section{References}

Anderson, E., Bai, Z., Bischof, C., Blackford, S., Demmel, J., Dongarra, J., Du Croz, J., Greenbaum, A., Hammarling, S., McKenney, A., and Sorensen, D. (1999). LAPACK Users' Guide. Society for Industrial and Applied Mathematics, Philadelphia, PA, third edition.

Banerjee, S., Gelfand, A., Finley, A., and Sang, H. (2008). Gaussian predictive process models for large spatial data sets. Journal of the Royal Statistical Society, Series B, 70(4):825-848.

Bates, D. and Maechler, M. (2015). Matrix: Sparse and Dense Matrix Classes and Methods. R package version 1.2-3.

Bayraktar, H. and Turalioglu, F. (2005). A kriging-based approach for locating a sampling site in the assessment of air quality. SERRA, 19(4):301 - 305.

Billings, S. D., Beatson, R. K., and Newsam, G. N. (2002a). Interpolation of geophysical data using continuous global surfaces. Geophysics, 67(6):1810 - 1822.

Billings, S. D., Beatson, R. K., and Newsam, G. N. (2002b). Smooth fitting of geophysical data using continuous global surfaces. Geophysics, 67(6):1823 - 1834.

Brown, R. and Hwang, P. (1992). Introduction to Random Signals and Applied Kalman Filtering. Wiley Series in Probability and Statistics, 2 edition.

Chiles, J. and Delfiner, P. (1999). Geostatistics, Modeling Spatial uncertainty. Wiley Series in Probability and statistics.

Cressie, N. (1993). Statistics for Spatial Data. Wiley Series in Probability and Mathematical Statistics, revised edition.

Cressie, N. and Johannesson, G. (2008). Fixed rank kriging for very large spatial data sets. Journal of the Royal Statistical Society B, 70(1):209 - 226. 
Davis, T. (2006). Direct Methods for Sparse Linear Systems. Fundamentals of Algorithms. Society for Industrial and Applied Mathematics.

Dempster, A. P., Laird, N. M., and Rubin, D. B. (1977). Maximum likelihood for incomplete data via the em algorithm. Jounal of the Royal Statistical Society, Series B, 39:1-38.

Finley, A., Sang, H., Banerjee, S., and Gelfand, A. (2008). Improving the performance of predictive process modeling for large datasets. Computational Statistics and Data Analysis, 53(8):2873-2884.

Fuentes, M. (2002). Spectral methods for nonstationary spatial processes. Biometrika, 89(1):197210.

Furrer, R., Genton, M., and Nychka, D. (2006). Covariance tapering for interpolation of large spatial datasets. Journal of Computational and Graphical Statistics, 15(3):502 - 523.

Golub, G. and Van Loan, C. (1996). Matrix Computations. Johns Hopkins, 3 edition.

Goovaerts, P. (1997). Geostatistics for natural resource evaluation. Springer.

Henderson, H. and Searle, S. (1981). On deriving the inverse of a sum of matrices. Society for Industrial and Applied Mathematics Review, 23(1):53 - 60.

Higdon, D. (1998). A process-convolution approach to modelling temperatures in the north atlantic ocean. Environmental and Ecological Statistics, 5(2):173-190.

Higham, N. (2002). Accuracy and Stability of Numerical Algorithms. Society for Industrial and Applied Mathematics, 2 edition.

Hoaglin, D., Mosteller, F., and Tukey, J. (1983). Understanding Robust and Exploratory Data Analysis. Wiley Series in Probability and Statistics.

Huang, H. C., Cressie, N., and Gabrosek, J. (2002). Fast, resolution-consistent spatial prediction of global processes from satellite data. Journal of Computational Graphical Statistics, 11:63 - 88.

Johannesson, G. and Cressie, N. (2004). Variance-covariance modeling and estimation for multiresolution spatial models. In Sanchez-Vila, X., Carrera, J., and Gomez-Hernandez, J., editors, geoENV IV-Geostatistics for Environmental Applications, pages 319-330. Dordrecht: Kluwer. 
Johannesson, G., Cressie, N., and Huang, H. C. (2007). Dynamic multi-resolution spatial models. Environmental and Ecological Statistics, 14:5-25.

Johns, C., Nychka, D., Kittel, T., and C., D. (2003). Infilling sparse records of spatial fields. Journal of the American Statistical Association, 98(464):796 - 806.

Katzfuss, M. (2016). A multi-resolution approximation for massive spatial datasets. Journal of the American Statistical Association, 0(ja).

Katzfuss, M. and Cressie, N. (2011). Spatio-temporal smoothing and em estimation for massive remote-sensing data sets. Journal of Time Series Analysis, 32:430-446.

Kaufman, C., Schervish, M., and Nychka, D. (2008). Covariance tapering for likelihood-based estimation in large spatial data sets. Journal of the American Statistical Association, 103(484):1545 1555.

Krige, D. (1951). A statistical approach to some basic mine valuation problems on the witwatersrand. Journal of the Chemical, Metallurgical and Mining Society of South Africa, 52(6):119-139.

Lindgren, F., Rue, H., and Lindström, J. (2011). An explicit link between Gaussian fields and Gaussian Markov random fields: the stochastic partial differential equation approach. Journal of the Royal Statistical Society: Series B (Statistical Methodology), 73(4):423-498.

Matheron, G. (1962). Traite de Geostatistique Applique, volume 1. Technip.

National Weather Service (2000). Cooperative Observer Program (COOP).

Nychka, D. (2000). Spatial-process estimates as smoothers. In Schimek, M. G., editor, Smoothing and Regression: Approaches, Computation, and Application, pages 393 - 424. New York: Wiley.

Nychka, D., Bailey, B., Ellner, S., Haaland, P., and O'Connell, M. (1996). FUNFITS: Data Analysis and Statistical Tools for Estimating Functions. Raleigh: North Carolina State University.

Nychka, D., Bandyopadhyay, S., Hammerling, D., Lindgren, F., and Sain, S. (2015a). A multiresolution Gaussian process model for the analysis of large spatial datasets. Journal of Computational and Graphical Statistics, 24(2):579-599. 
Nychka, D., Furrer, R., Paige, J., and Sain, S. (2015b). fields: Tools for Spatial Data. R package version 8.3-5.

Nychka, D., Wikle, C., and Royle, J. A. (2002). Multiresolution models for nonstationary spatial covariance functions. Statistical Modelling, 2(4):315 - 331.

Peterson, K. and Pedersen, M. (2008). The Matrix Cookbook.

Quiñero Candela, J. and Rasmussen, C. (2005). A unifying view of sparse approximate Gaussian process regression. Journal of Machine Learning Research, 6:1939 - 1959.

Richmond, A. (2003). Financially efficient ore selection incorporating grade uncertainty. Mathematical Geology, 35(2):195 - 215.

Sacks, J., Welch, W., Mitchell, T., and Wynn, H. (1989). Design and analysis of computer experiments. Statistical Science, 4(4):409 - 435.

Searle, S. (2006). Matrix Algebra Useful for Statistics. Wiley Series in Probability and Statistics.

Stein, A., van der Meer, F., and Gorte, B. (2002). Spatial Statistics for remote sensing. Kluwer Academic Publishers.

Stein, M. (1999). Interpolation of Spatial Data. Springer.

Stein, M., Chi, Z., and Welty, L. (2004). Approximating likelihoods for large spatial data sets. Journal of the Royal Statistical Society: Series B (Statistical Methodology), 66(2):275-296.

Vecchia, A. (1988). Estimation and model identification for continuous spatial processes. Journal of the Royal Statistical Society.Series B (Methodological), pages 297-312. 


\title{
Supplement to "Reduced Basis Kriging for Massive Spatial Fields"
}

\author{
Karl Pazdernik ${ }^{1}$, Ranjan Maitra ${ }^{1}$, Douglas Nychka ${ }^{2}$, and Stephan Sain ${ }^{2}$ \\ ${ }^{1}$ Department of Statistics and Statistical Laboratory, Iowa State University \\ ${ }^{2}$ Institute for Mathematics Applied to Geosciences, National Center for \\ Atmospheric Research
}

\section{S-1 Additional Experimental Evaluations}

A simulation study was conducted to assess the value of reduced basis kriging in comparison to obtaining maximum likelihood estimates of the Spatial Random Effects model via the E-M algorithm. One hundred 2-dimensional Gaussian random fields were simulated using the $\mathrm{R}$ package fields (Nychka et al., 2015) on either a $50 \times 50$ or $200 \times 200$ grid. The spatial process at the locations of the observations were assumed to follow a Gaussian distribution with a Matern covariance structure. The Matérn parameter values $\nu$ and $\theta$ were varied in unison to be such that $(\nu, \theta) \in\{(0.5,0.205),(1,0.137),(1.5,0.110),(2,0.095)\}$, holding $\rho=1$ constant. For each field, simulated measurement errors $\left(\sigma^{2}=\{0,0.1,0.25,0.4\}\right)$ were added to each response at 300 randomly selected locations, resulting in values that represented data.

ML estimates and the corresponding predicted values for each simulated field were then computed using three different methods: reduced basis kriging using an identity covariance for $\boldsymbol{K}=\delta \boldsymbol{I}$ (blue), by the E-M approach approximating $\boldsymbol{K}$ with the identity matrix, $\delta \boldsymbol{I}$ (gold), and by the E-M approach using the full form of $\boldsymbol{K}$ with constant $b=1.5$ (white). Basis functions with a single resolution and either 23,77 , or 175 knots were used during estimation and prediction, with the bandwidth constant allowed to vary from 0.5 to 2.5 by 0.1 for both methods that use the identity covariance. As mentioned in the full paper, these computations were done using sparse matrix methods (Davis, 2006) implemented as per the R package "Matrix" (Bates and Maechler, 2015).

The value of reduced basis kriging was quantified by two summaries: the time required to iterate to convergence followed by computing kriging estimates and the mean square prediction error of the predicted field. The distributions of the one hundred simulated fields are represented with boxplots on the log scale in Figures S-1 through S-16. For clarity of presentation, the distribution of seconds or MSPE for the E-M approach using the full form of $\boldsymbol{K}$ are truncated on the main figure and presented in full separately when the maximum is significantly beyond the range of the corresponding results for the identity form of $\boldsymbol{K}$, as in Figure S-1(c).

Two expected gains in computational efficiency were achieved by either using the identity covariance for $\boldsymbol{K}$ or by using fewer knots. The value of the bandwidth constant, $b$, was also critical to minimizing CPU time because it controlled the level of sparsity in $S$. The effect of $b$ on the E$\mathrm{M}$ approach was predictable as lower values resulted in decreased CPU time, with an optimal level 
of sparsity occurring near $b=1$ for the $50 \times 50$ grid. An interesting difference between the E-M approach and reduced basis kriging is the robustness of reduced basis kriging to increasing $m$ or a poor choice of bandwidth constant. Reduced basis kriging is efficient regardless of the specifics of the basis functions used and is at least as efficient as the E-M approach with the identity covariance for any reasonable choice of $b$. The E-M approach is consistently more computationally expensive for larger values of $b$. When the $200 \times 200$ grid size was used, the E-M approach would occasionally reach convergence sooner than reduced basis kriging for low values of the bandwidth constant $(b<1)$, with a dramatic reduction occurring when $b=0.5$ and $m=23$. However, the resulting MSPE of these estimations were particularly poor.

The distributions of MSPE for both $50 \times 50$ and $200 \times 200$ grid sizes show that for a reasonable $b$, the identity covariance structure is at least as accurate in terms of prediction as the full covariance structure. In fact, as $m$ approaches $n$, the identity covariance performs substantially better than the full covariance with regards to both median and maximum MSPE. This suggests that added complexity to $\boldsymbol{K}$ is unnecessary when our focus is on prediction. Using the identity covariance for $\boldsymbol{K}$, reduced basis kriging and the E-M approach are comparable for choices of bandwidth constant where MSPE is minimized, however an unreasonable $b$ favors the E-M approach in terms of MSPE. This is opposite of computational efficiency, in which reduced basis kriging was more robust to poor choices of $b$. 


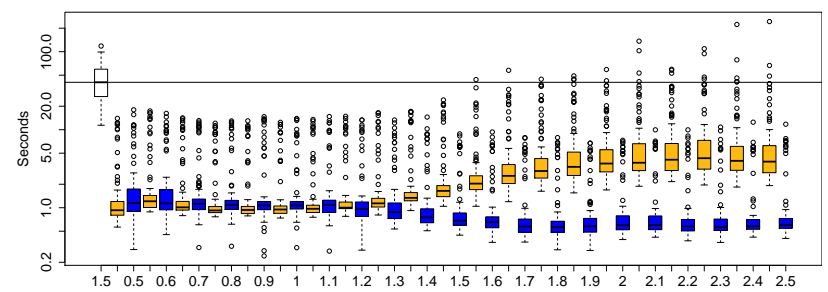

(a) $50 \times 50$ grid with $m=23$

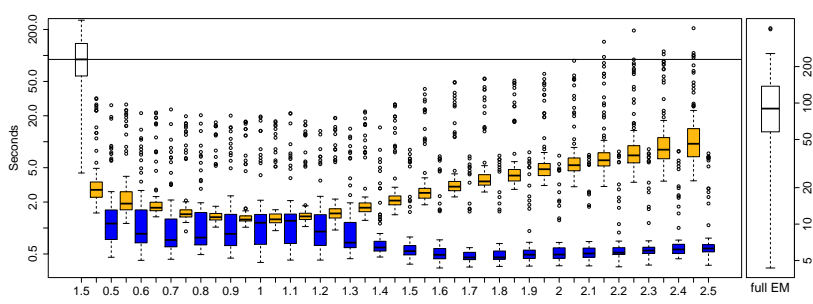

(c) $50 \times 50$ grid with $m=77$

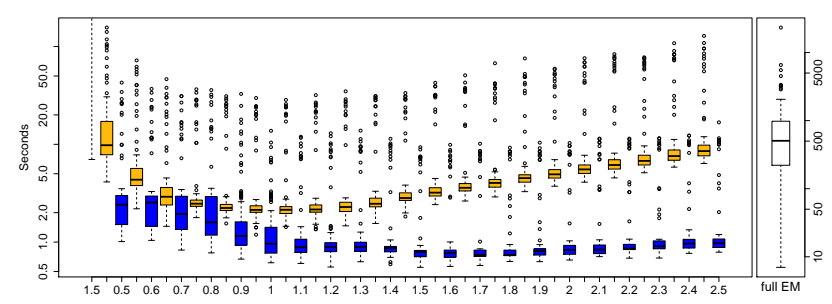

(e) $50 \times 50$ grid with $m=175$

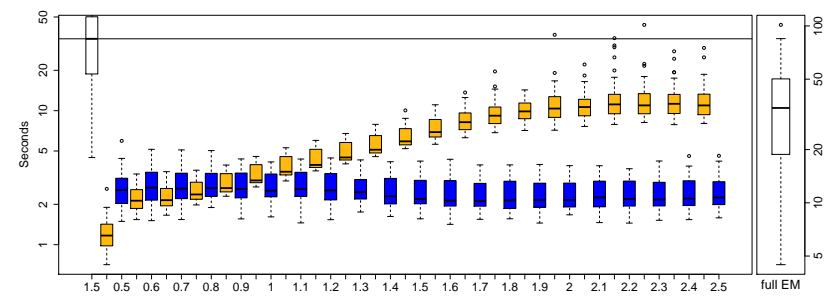

(g) $200 \times 200$ grid with $m=23$

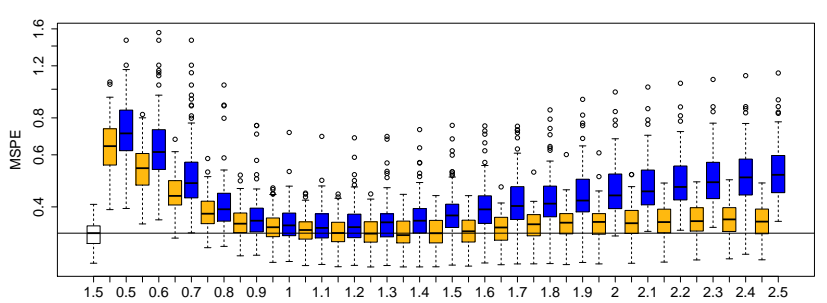

(b) $50 \times 50$ grid with $m=23$

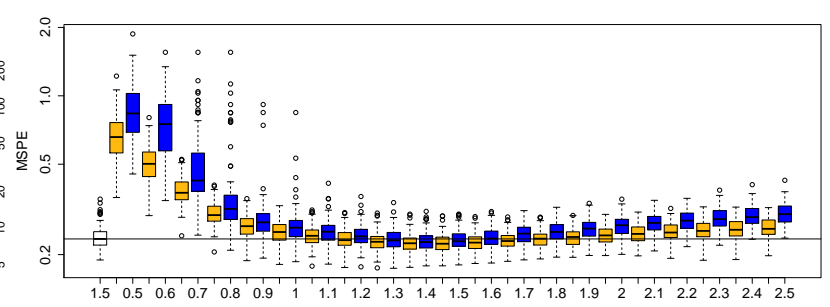

(d) $50 \times 50$ grid with $m=77$

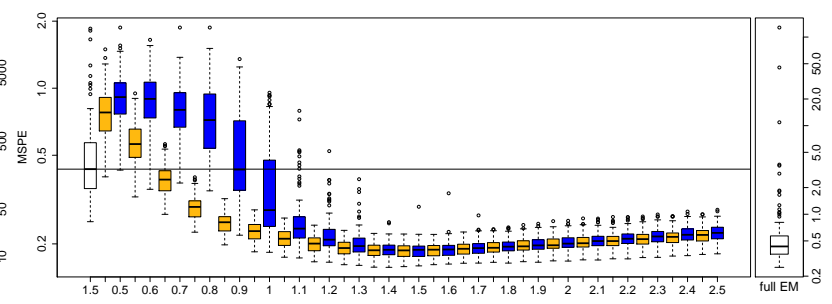

(f) $50 \times 50$ grid with $m=175$

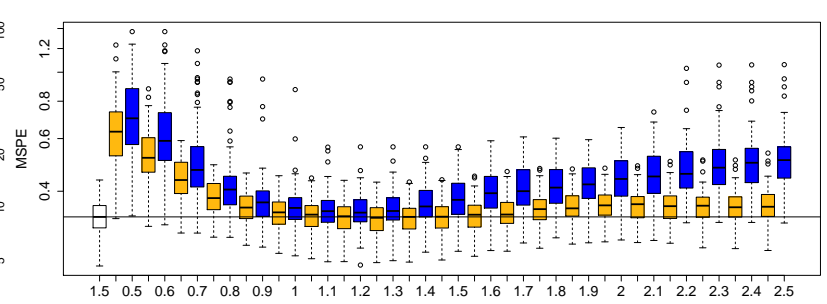

(h) $200 \times 200$ grid with $m=23$

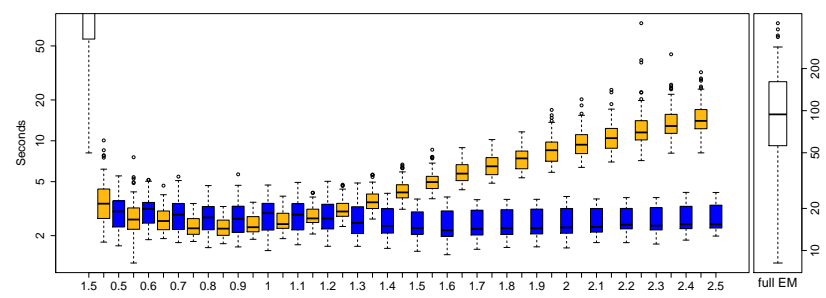

(i) $200 \times 200$ grid with $m=77$

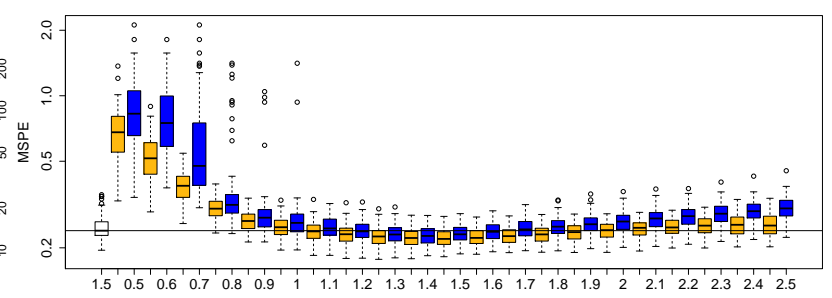

(j) $200 \times 200$ grid with $m=77$

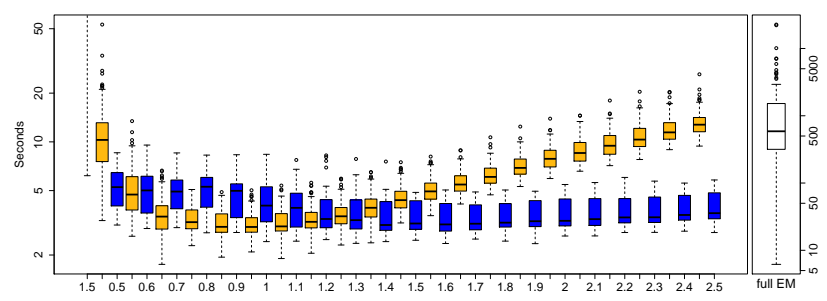

(k) $200 \times 200$ grid with $m=175$

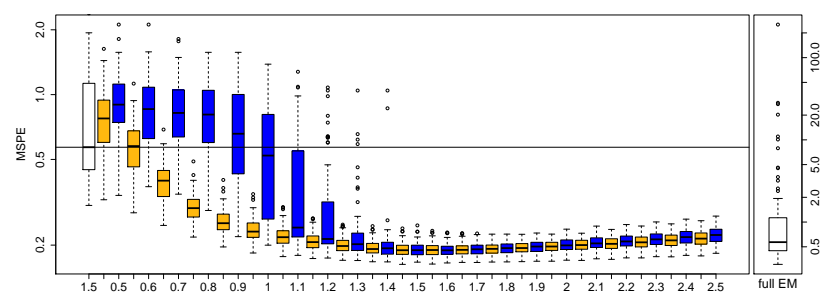

(l) $200 \times 200$ grid with $m=175$

Figure S-1: Seconds (left panel) and Mean SquaredzPrediction Errors (MSPEs, right panel) for varying bandwidths constants (x-axis) and number of knots $(m)$ for all three estimation methods on both grids when $\nu=0.5$ and $\sigma^{2}=0$. 


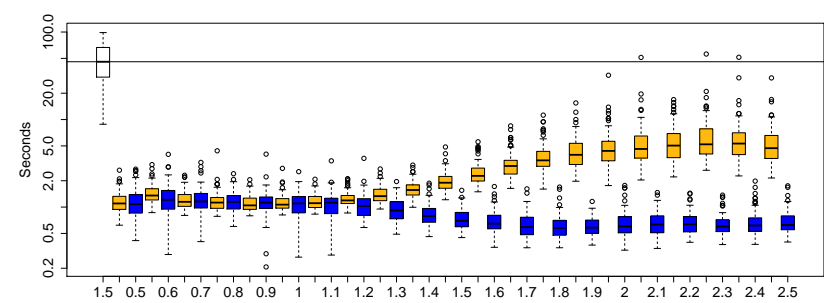

(a) $50 \times 50$ grid with $m=23$

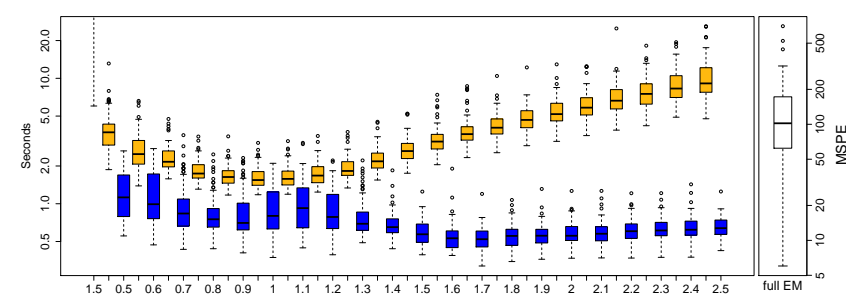

(c) $50 \times 50$ grid with $m=77$

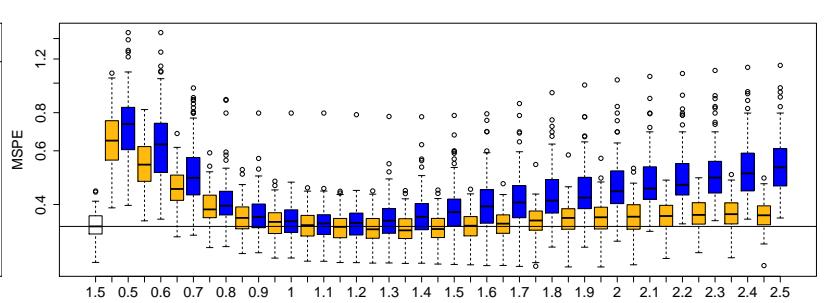

(b) $50 \times 50$ grid with $m=23$

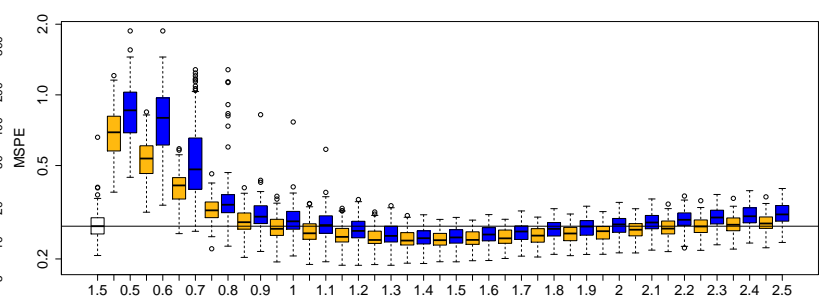

(d) $50 \times 50$ grid with $m=77$

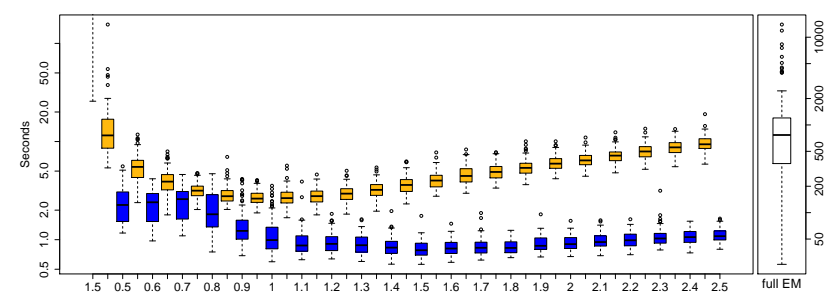

(e) $50 \times 50$ grid with $m=175$

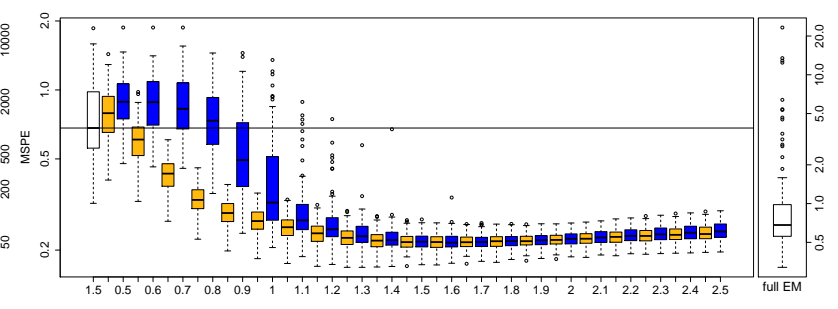

(f) $50 \times 50$ grid with $m=175$

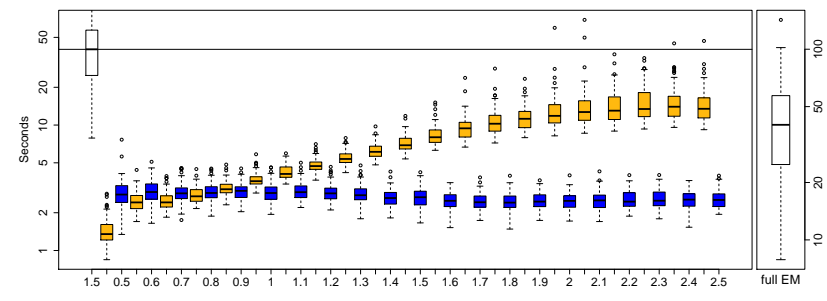

(g) $200 \times 200$ grid with $m=23$

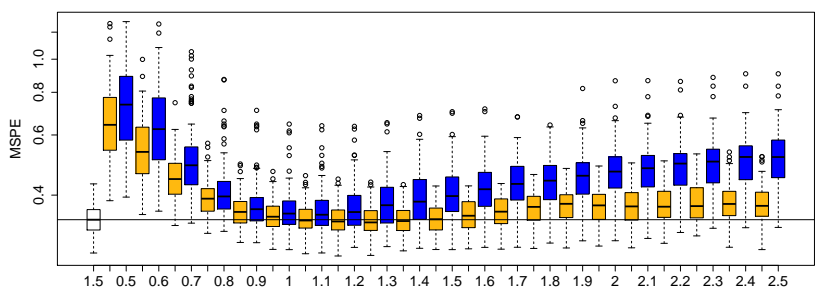

(h) $200 \times 200$ grid with $m=23$

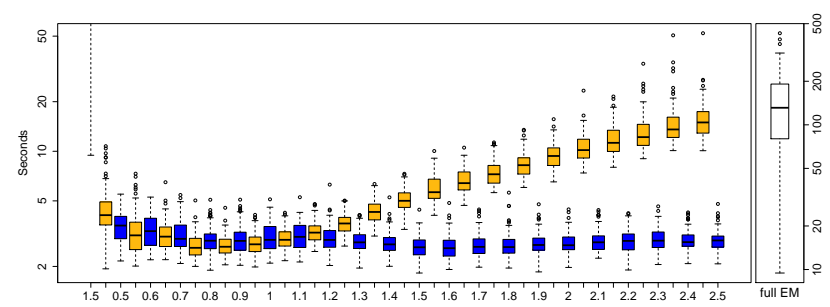

(i) $200 \times 200$ grid with $m=77$

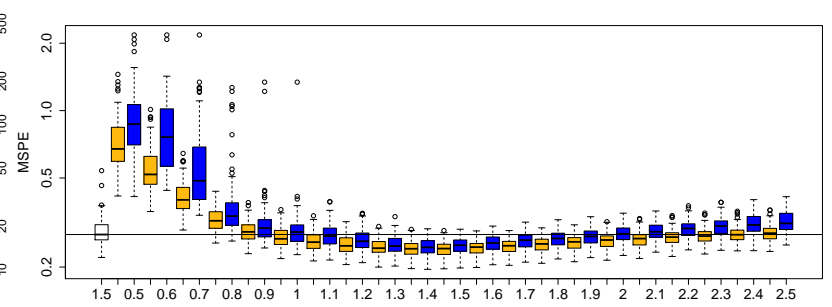

(j) $200 \times 200$ grid with $m=77$

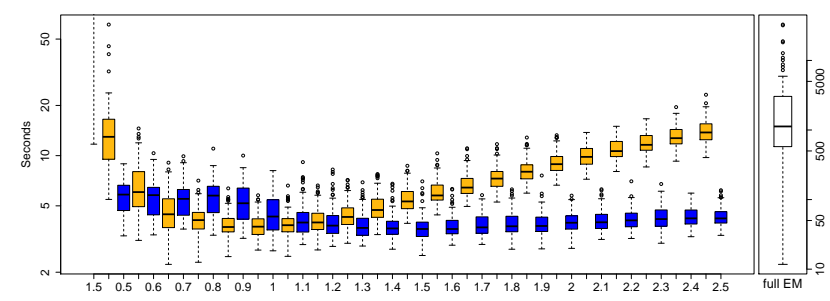

(k) $200 \times 200$ grid with $m=175$

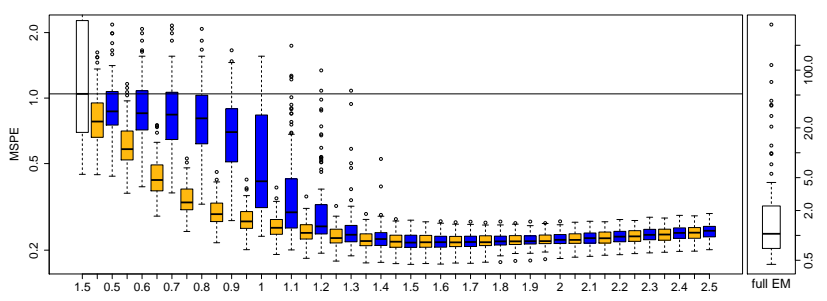

(l) $200 \times 200$ grid with $m=175$

Figure S-2: Seconds (left panel) and Mean Squared Prediction Errors (MSPEs, right panel) for varying bandwidths constants (x-axis) and number of knots $(m)$ for all three estimation methods on both grids when $\nu=0.5$ and $\sigma^{2}=0.1$. 


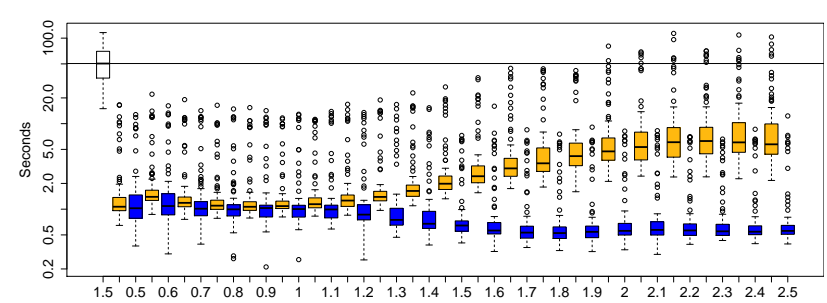

(a) $50 \times 50$ grid with $m=23$

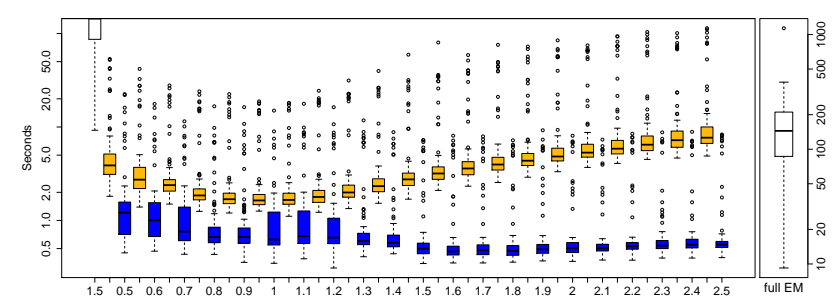

(c) $50 \times 50$ grid with $m=77$

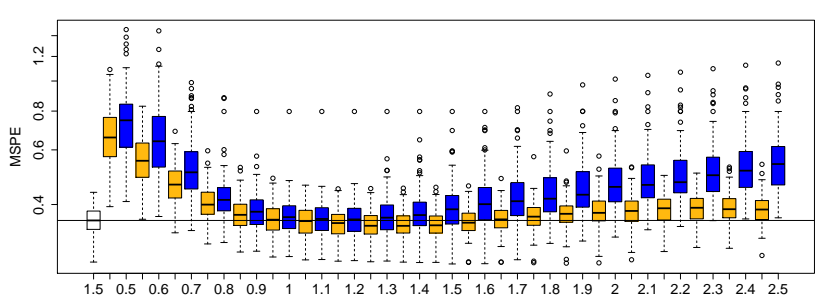

(b) $50 \times 50$ grid with $m=23$

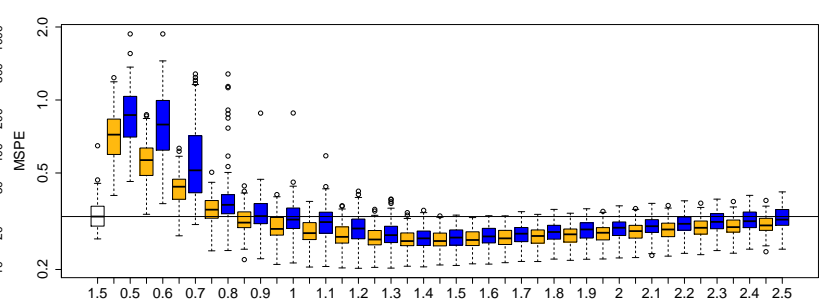

(d) $50 \times 50$ grid with $m=77$

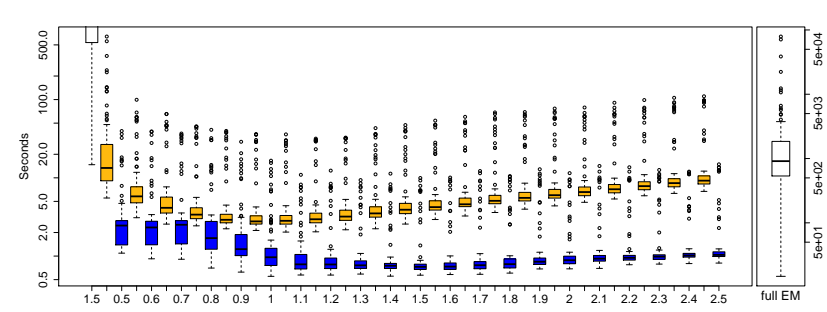

(e) $50 \times 50$ grid with $m=175$

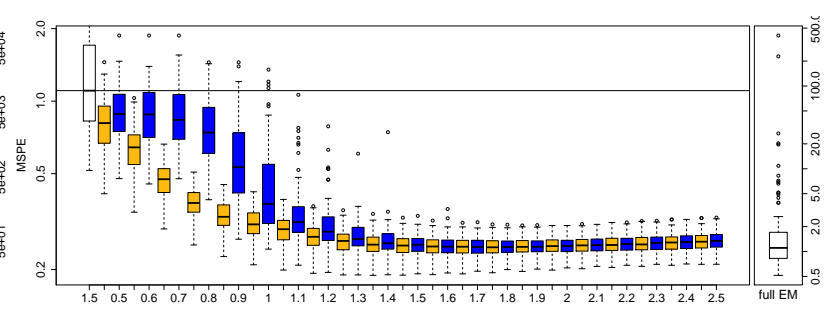

(f) $50 \times 50$ grid with $m=175$

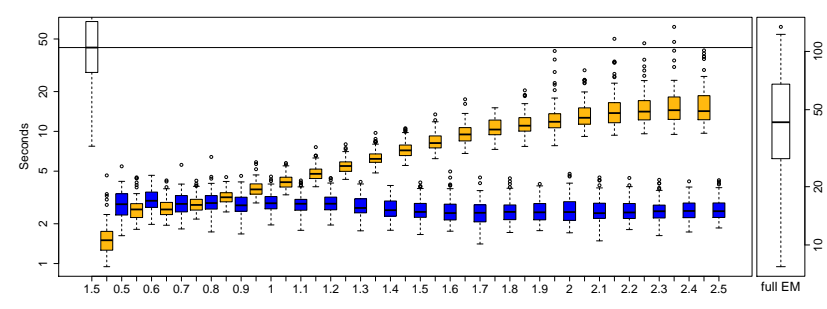

(g) $200 \times 200$ grid with $m=23$

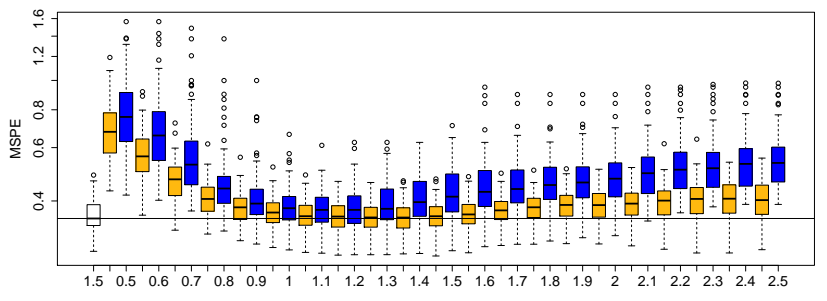

(h) $200 \times 200$ grid with $m=23$

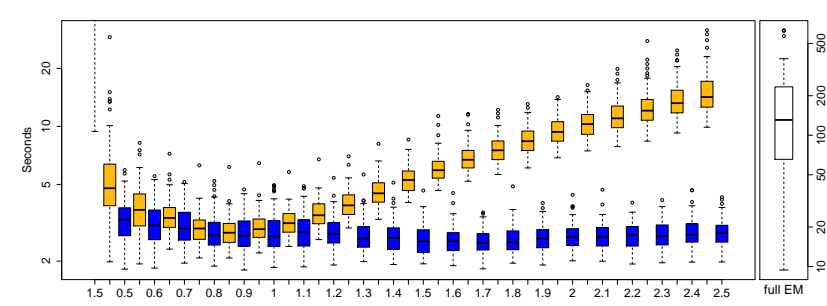

(i) $200 \times 200$ grid with $m=77$

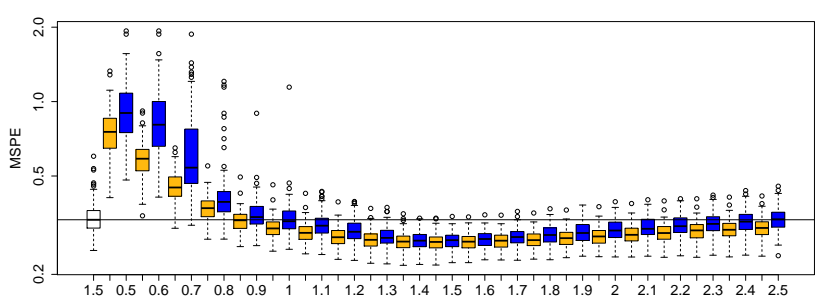

(j) $200 \times 200$ grid with $m=77$

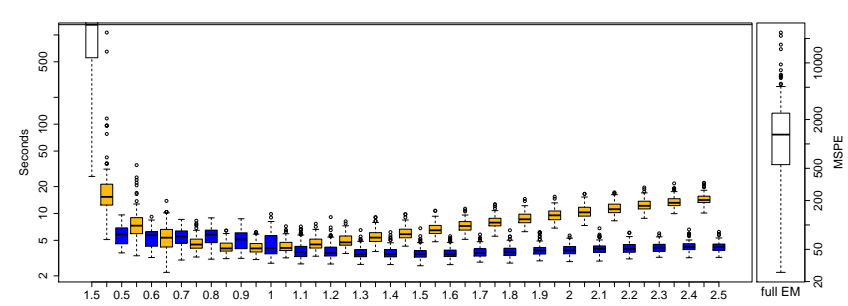

(k) $200 \times 200$ grid with $m=175$

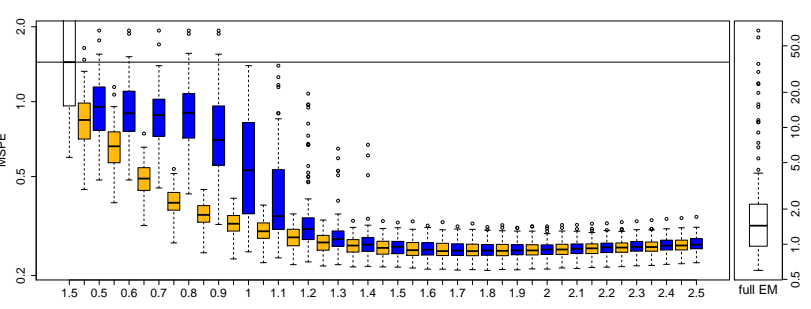

(l) $200 \times 200$ grid with $m=175$

Figure S-3: Seconds (left panel) and Mean SquaredfPrediction Errors (MSPEs, right panel) for varying bandwidths constants (x-axis) and number of knots $(m)$ for all three estimation methods on both grids when $\nu=0.5$ and $\sigma^{2}=0.25$. 


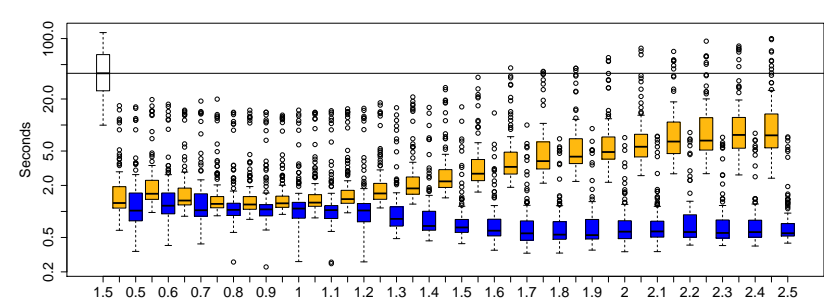

(a) $50 \times 50$ grid with $m=23$

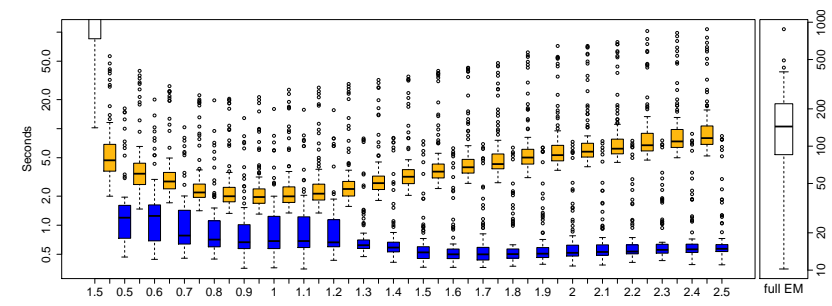

(c) $50 \times 50$ grid with $m=77$

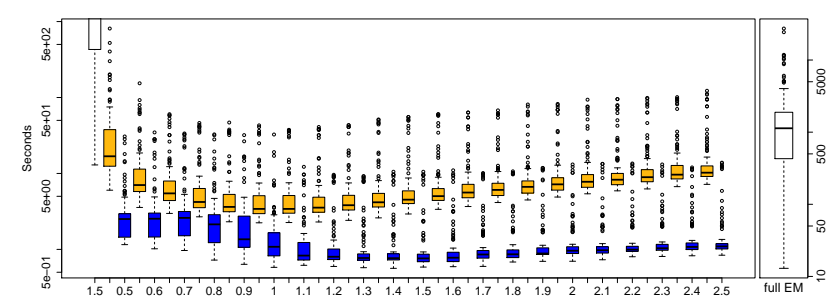

(e) $50 \times 50$ grid with $m=175$

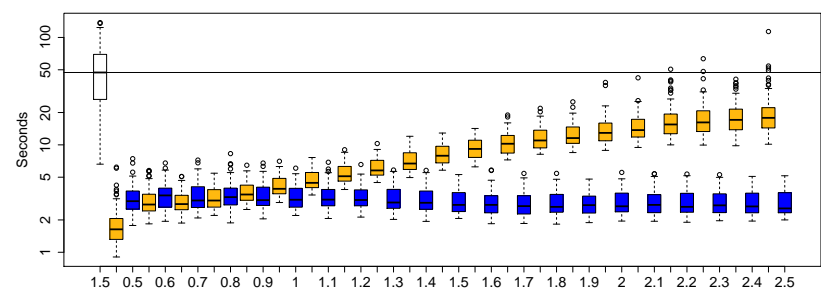

(g) $200 \times 200$ grid with $m=23$

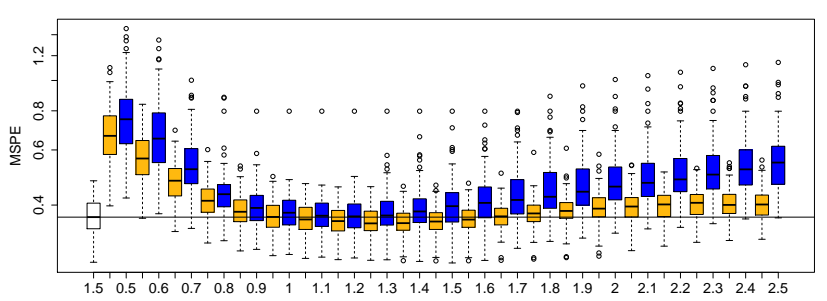

(b) $50 \times 50$ grid with $m=23$

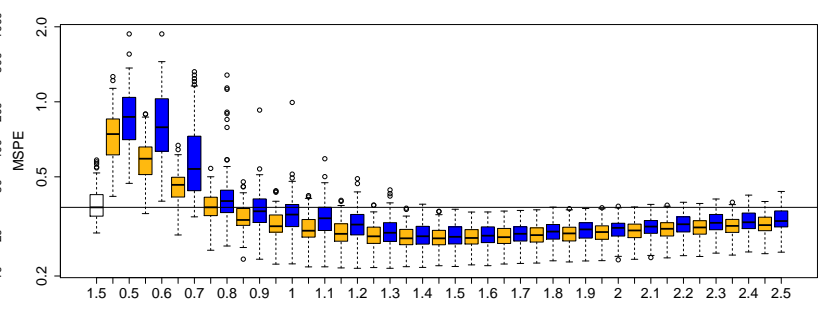

(d) $50 \times 50$ grid with $m=77$

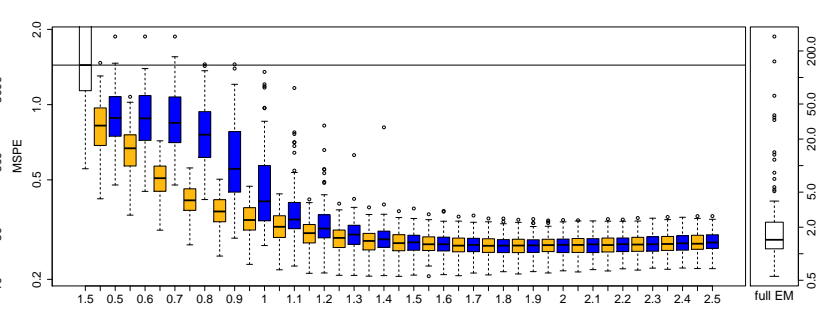

(f) $50 \times 50$ grid with $m=175$

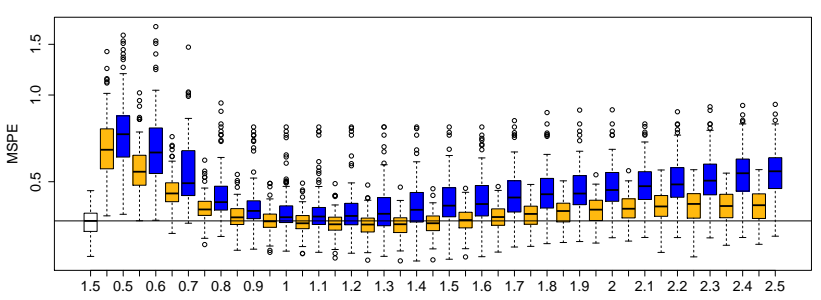

(h) $200 \times 200$ grid with $m=23$

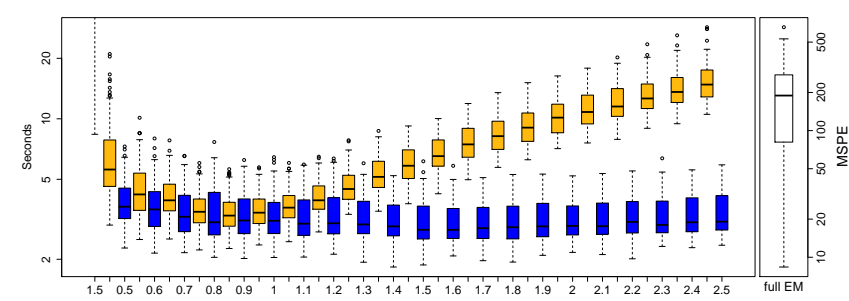

(i) $200 \times 200$ grid with $m=77$

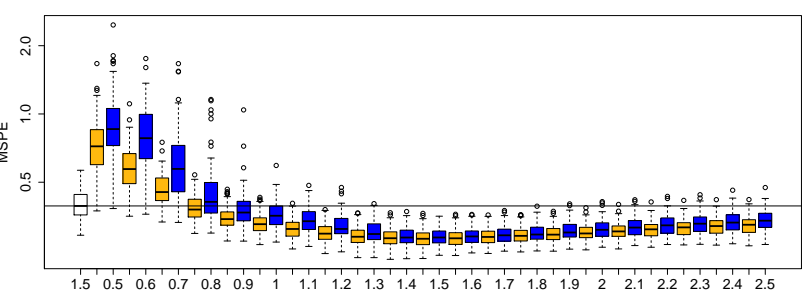

(j) $200 \times 200$ grid with $m=77$

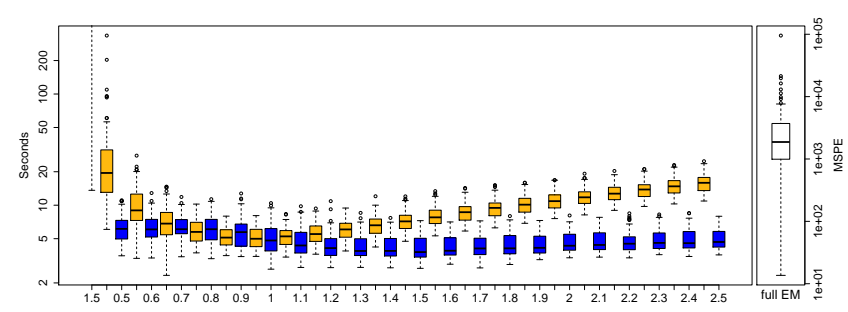

(k) $200 \times 200$ grid with $m=175$

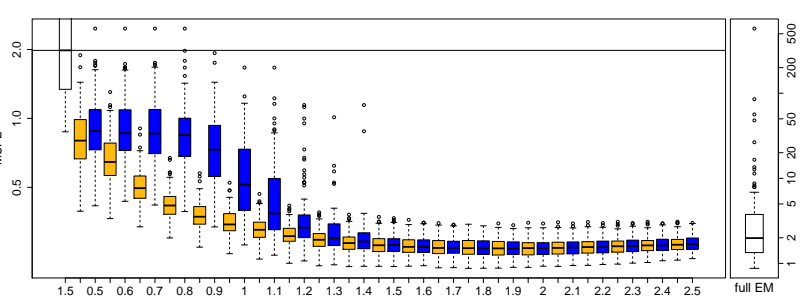

(l) $200 \times 200$ grid with $m=175$

Figure S-4: Seconds (left panel) and Mean SquaredfPrediction Errors (MSPEs, right panel) for varying bandwidths constants (x-axis) and number of knots $(m)$ for all three estimation methods on both grids when $\nu=0.5$ and $\sigma^{2}=0.4$. 


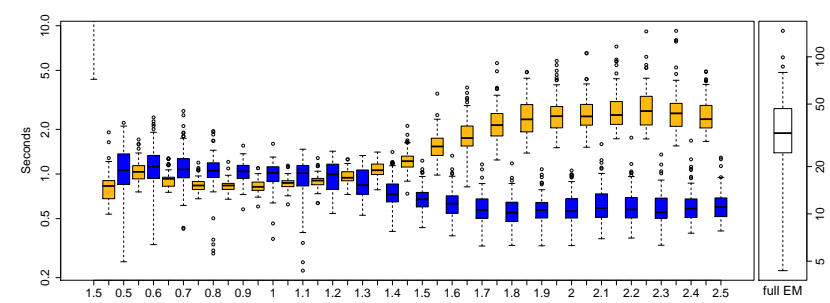

(a) $50 \times 50$ grid with $m=23$

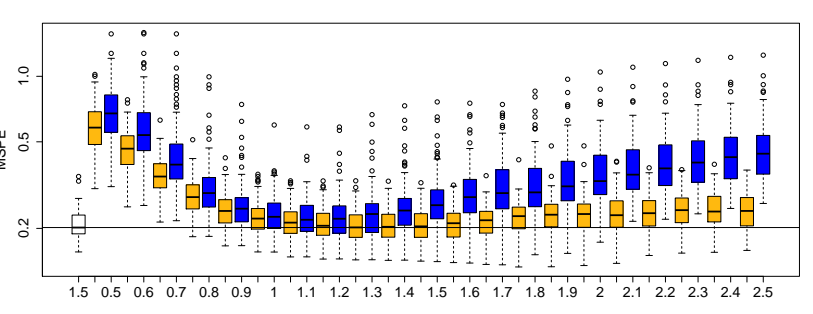

(b) $50 \times 50$ grid with $m=23$

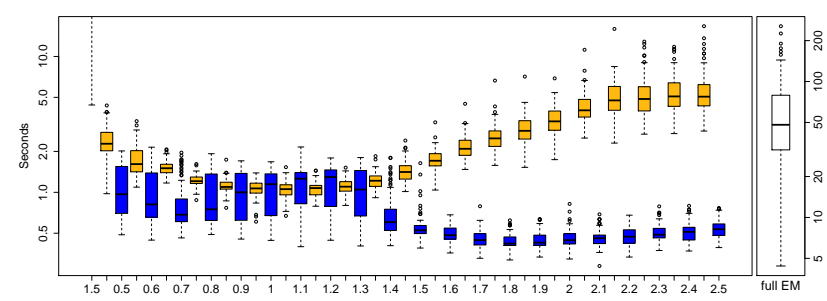

(c) $50 \times 50$ grid with $m=77$

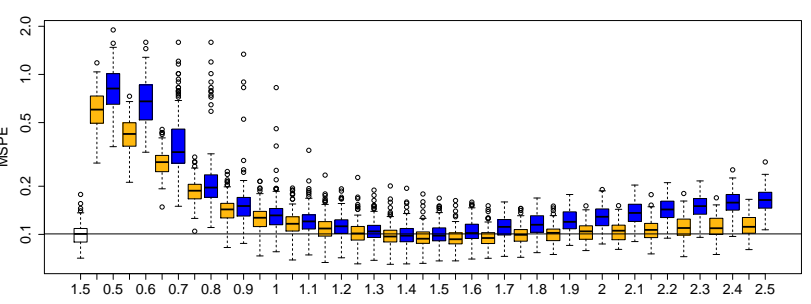

(d) $50 \times 50$ grid with $m=77$

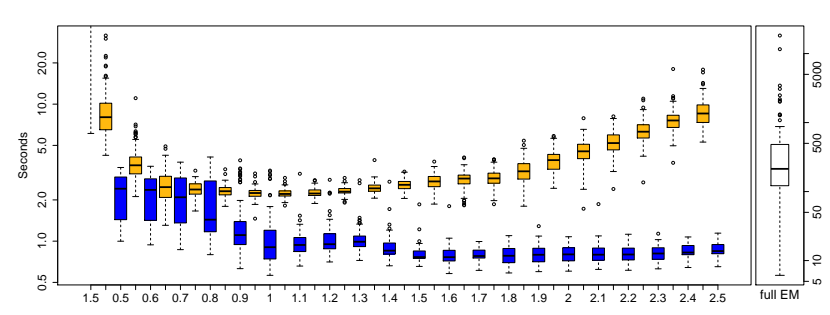

(e) $50 \times 50$ grid with $m=175$

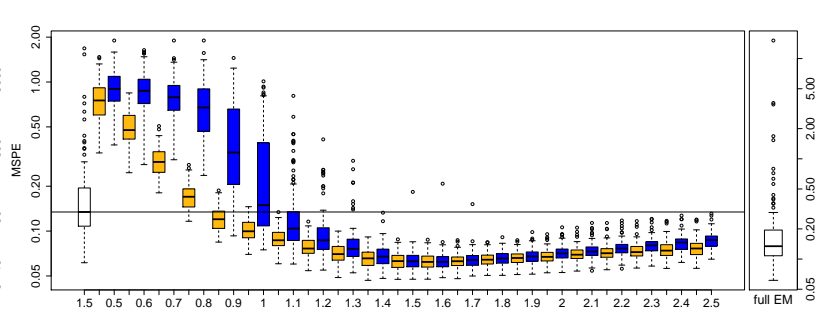

(f) $50 \times 50$ grid with $m=175$

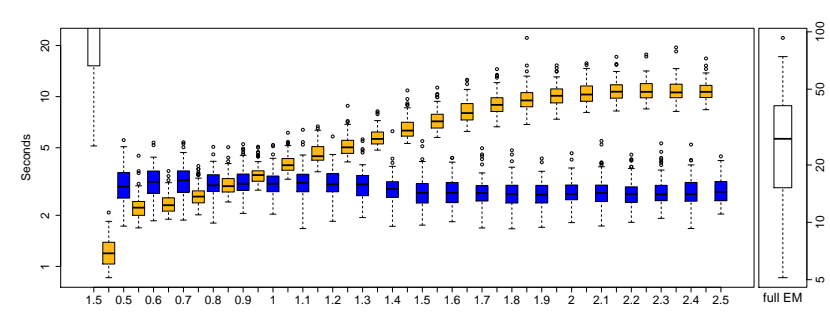

(g) $200 \times 200$ grid with $m=23$

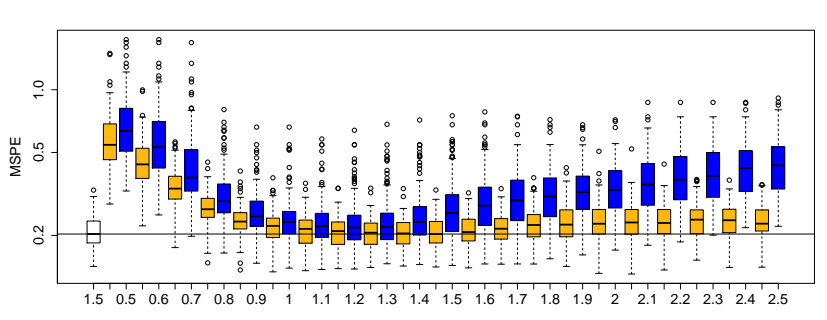

(h) $200 \times 200$ grid with $m=23$

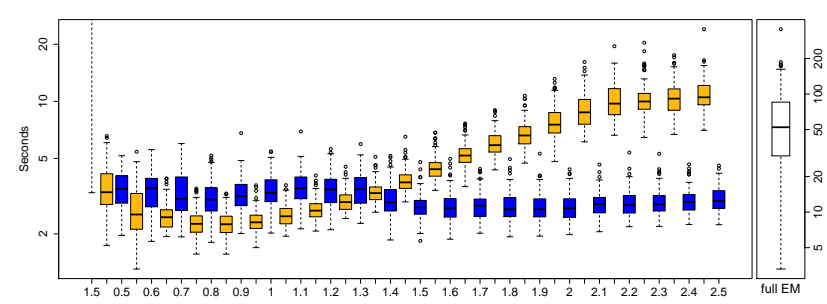

(i) $200 \times 200$ grid with $m=77$

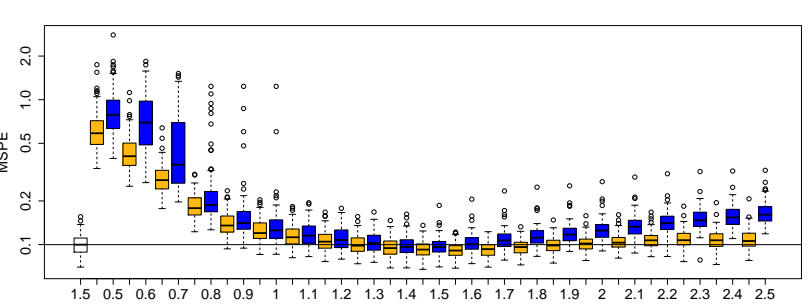

(j) $200 \times 200$ grid with $m=77$

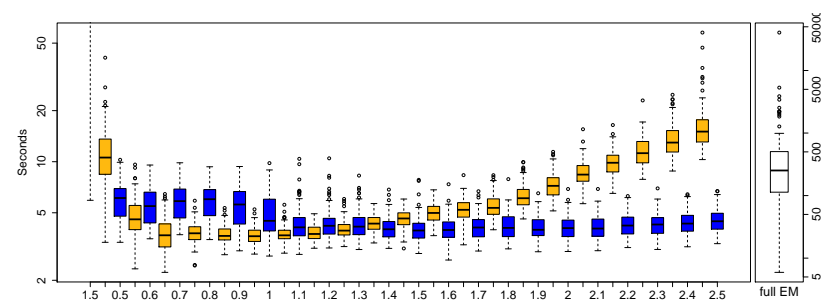

(k) $200 \times 200$ grid with $m=175$

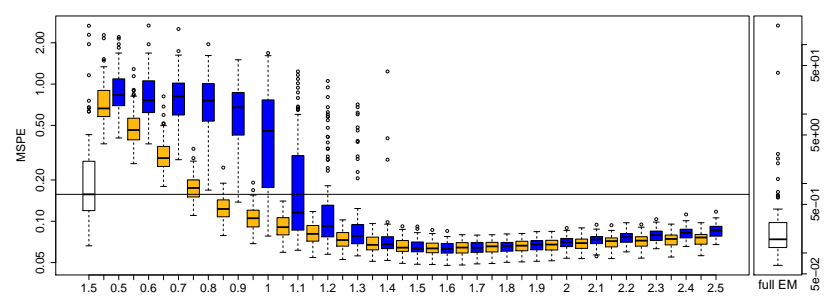

(l) $200 \times 200$ grid with $m=175$

Figure S-5: Seconds (left panel) and Mean Squaredprediction Errors (MSPEs, right panel) for varying bandwidths constants (x-axis) and number of knots $(m)$ for all three estimation methods on both grids when $\nu=1$ and $\sigma^{2}=0$. 


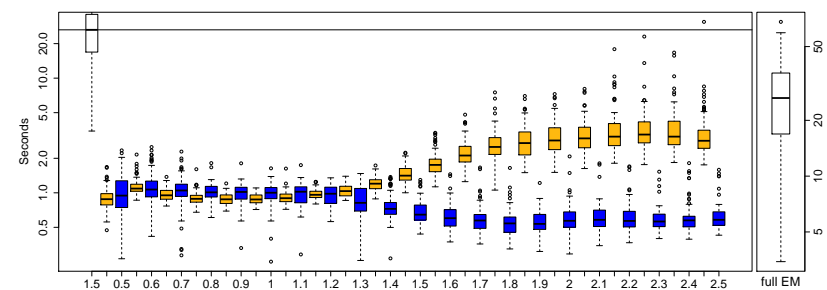

(a) $50 \times 50$ grid with $m=23$

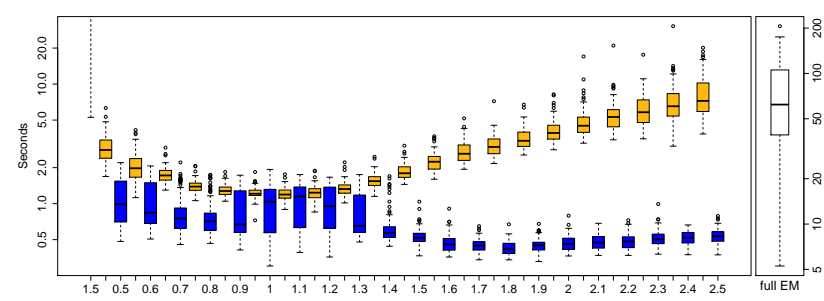

(c) $50 \times 50$ grid with $m=77$

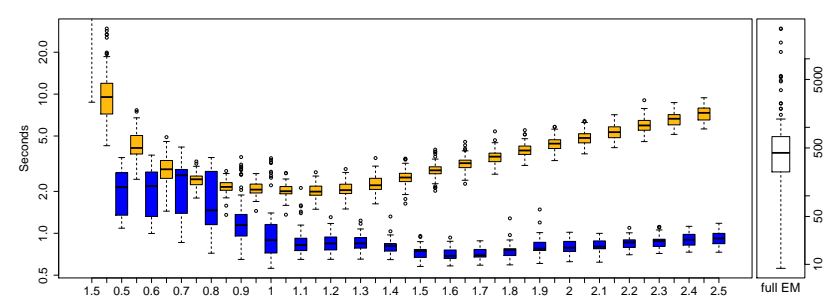

(e) $50 \times 50$ grid with $m=175$

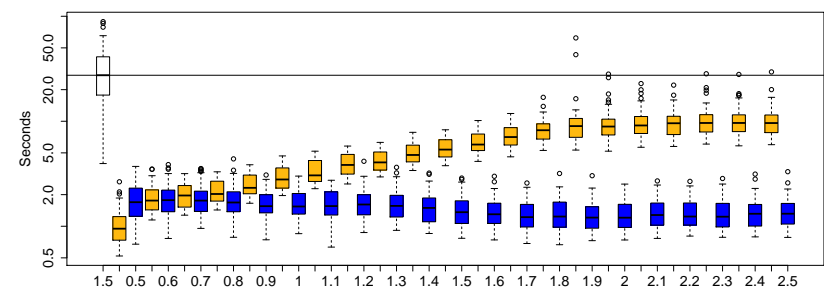

(g) $200 \times 200$ grid with $m=23$

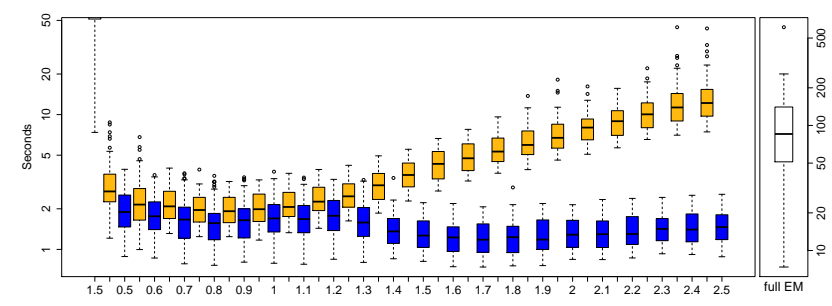

(i) $200 \times 200$ grid with $m=77$

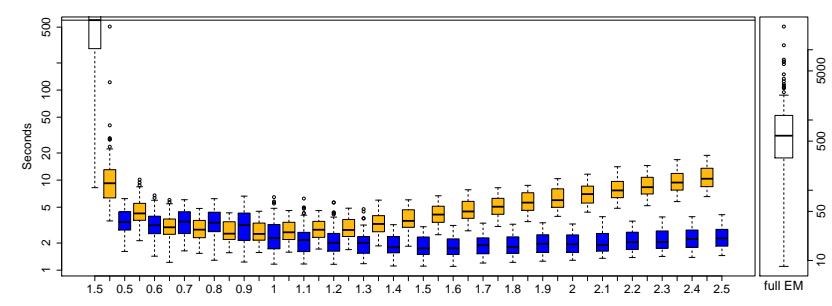

(k) $200 \times 200$ grid with $m=175$

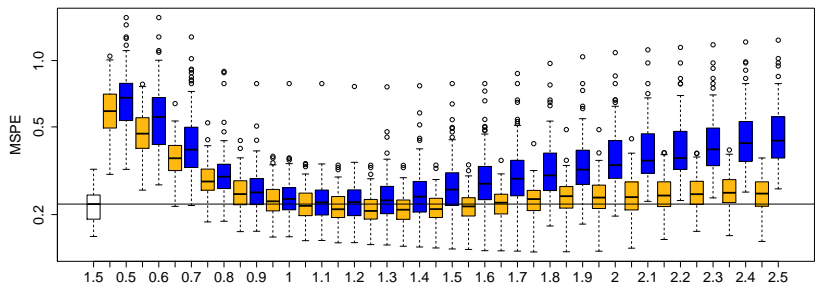

(b) $50 \times 50$ grid with $m=23$

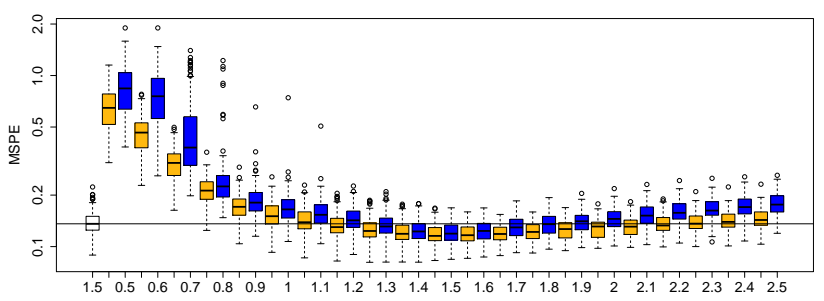

(d) $50 \times 50$ grid with $m=77$

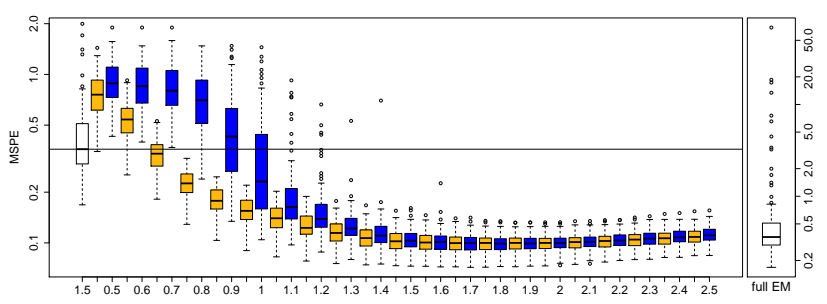

(f) $50 \times 50$ grid with $m=175$

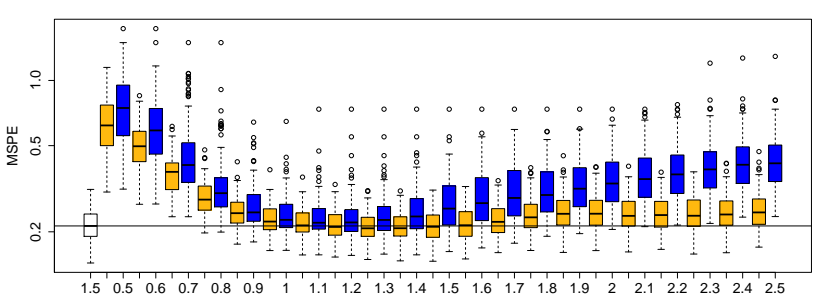

(h) $200 \times 200$ grid with $m=23$

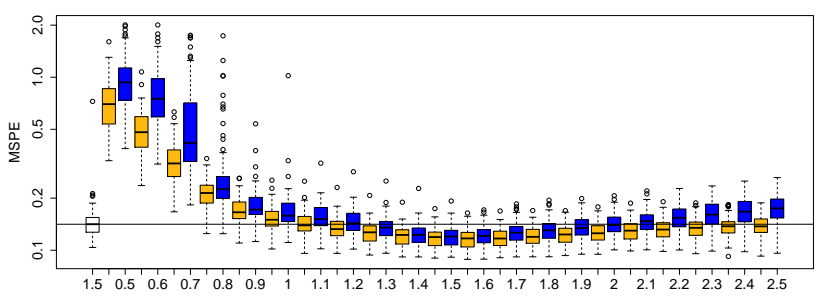

(j) $200 \times 200$ grid with $m=77$

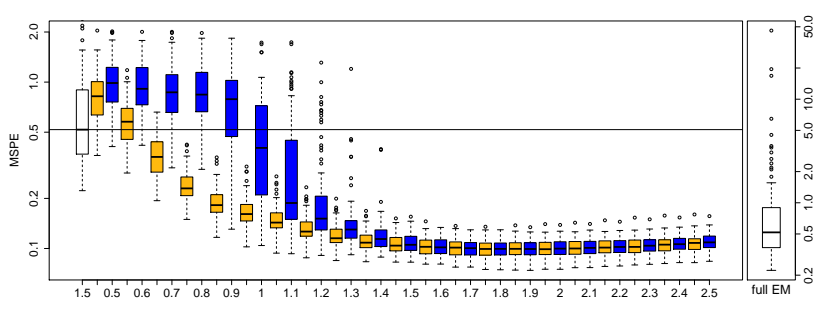

(l) $200 \times 200$ grid with $m=175$

Figure S-6: Seconds (left panel) and Mean Squared_Prediction Errors (MSPEs, right panel) for varying bandwidths constants (x-axis) and number of knots $(m)$ for all three estimation methods on both grids when $\nu=1$ and $\sigma^{2}=0.1$. 


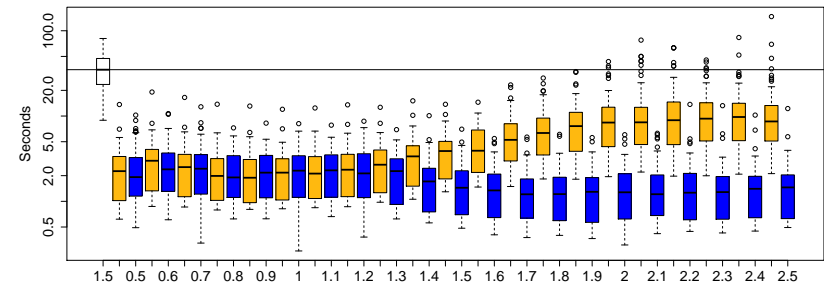

(a) $50 \times 50$ grid with $m=23$

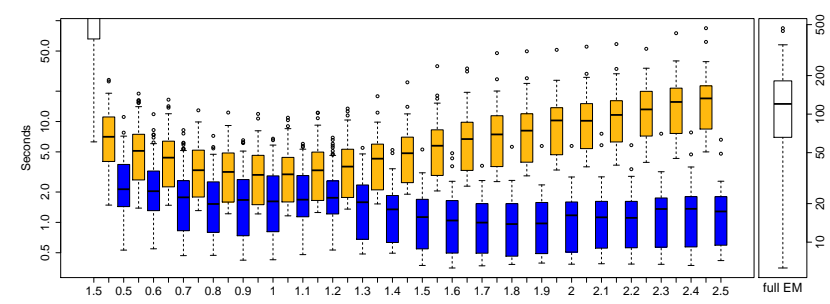

(c) $50 \times 50$ grid with $m=77$

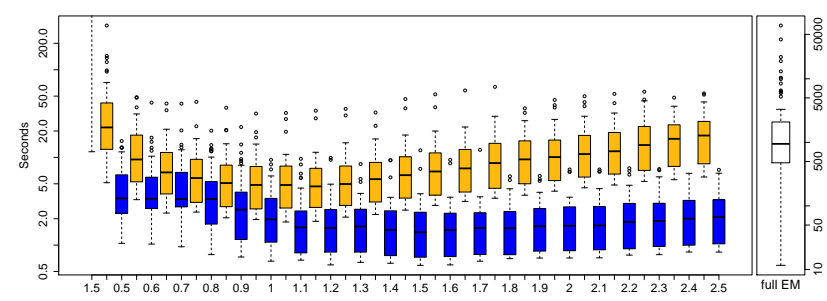

(e) $50 \times 50$ grid with $m=175$

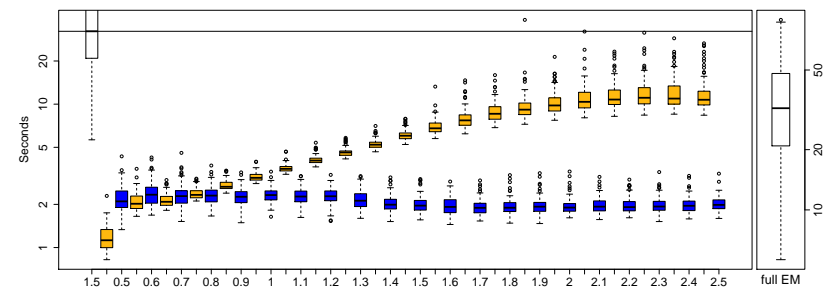

(g) $200 \times 200$ grid with $m=23$

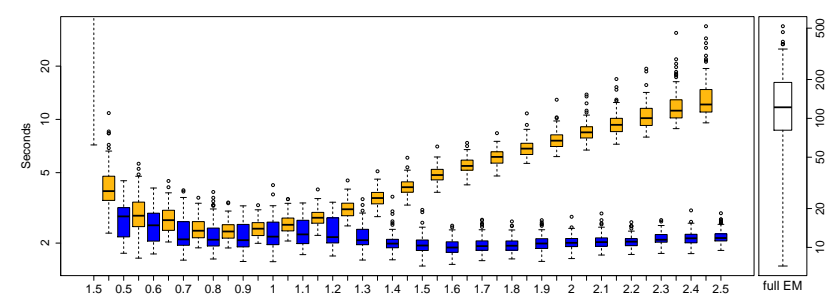

(i) $200 \times 200$ grid with $m=77$

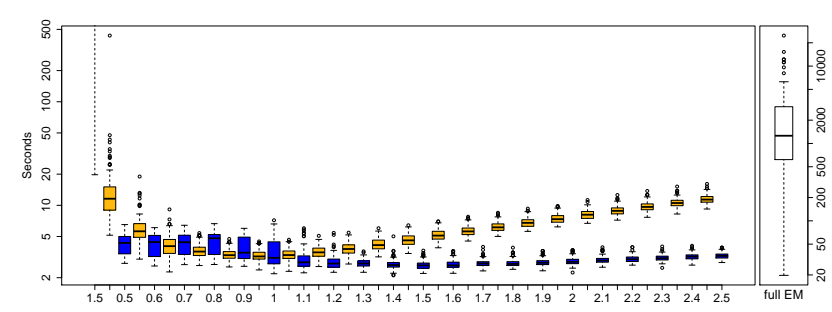

(k) $200 \times 200$ grid with $m=175$

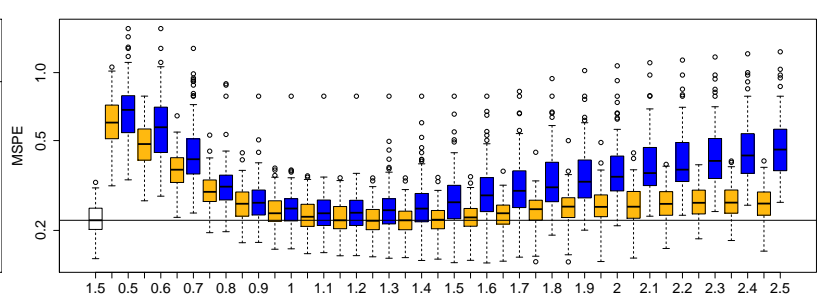

(b) $50 \times 50$ grid with $m=23$

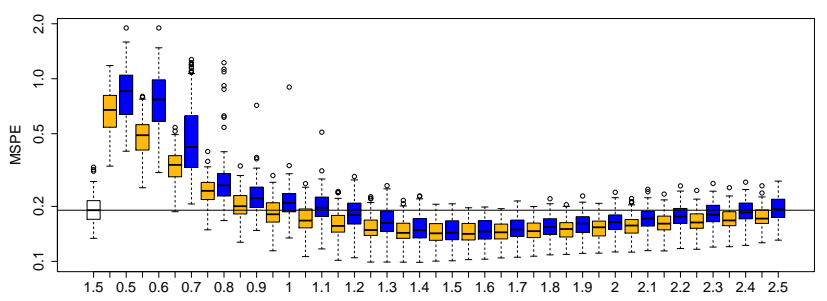

(d) $50 \times 50$ grid with $m=77$

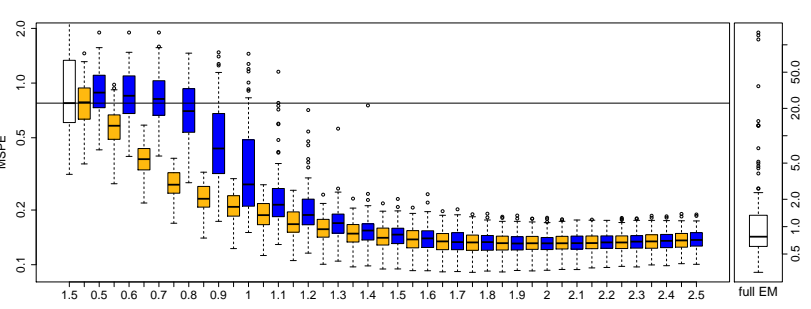

(f) $50 \times 50$ grid with $m=175$

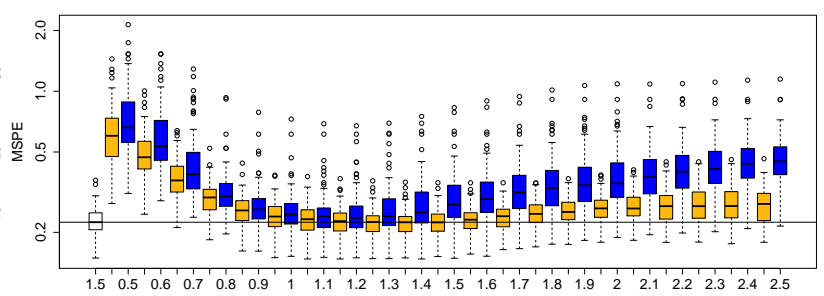

(h) $200 \times 200$ grid with $m=23$

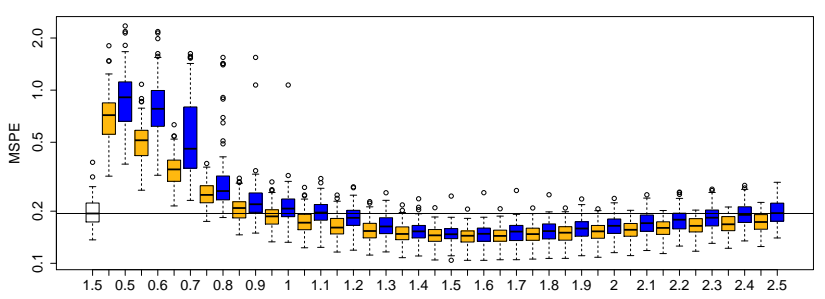

(j) $200 \times 200$ grid with $m=77$

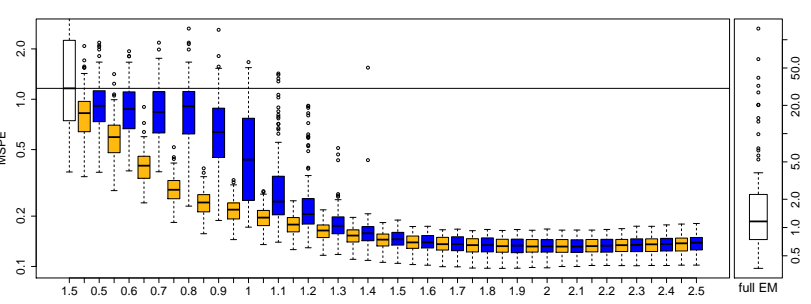

(l) $200 \times 200$ grid with $m=175$

Figure S-7: Seconds (left panel) and Mean Squaredprediction Errors (MSPEs, right panel) for varying bandwidths constants (x-axis) and number of knots $(m)$ for all three estimation methods on both grids when $\nu=1$ and $\sigma^{2}=0.25$. 


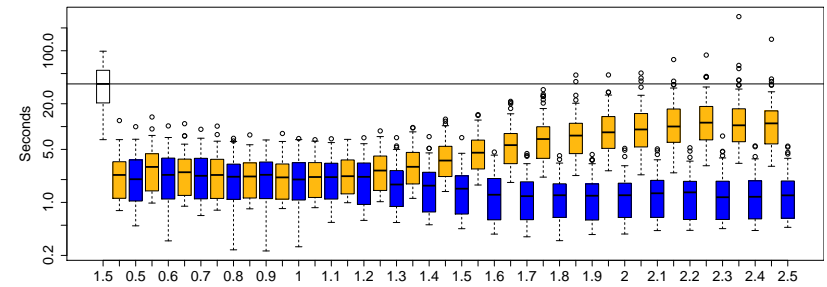

(a) $50 \times 50$ grid with $m=23$

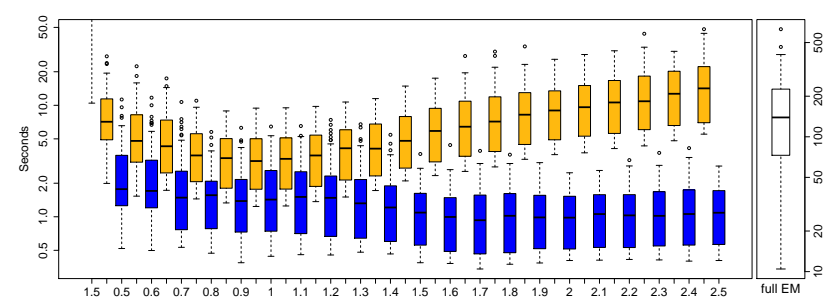

(c) $50 \times 50$ grid with $m=77$

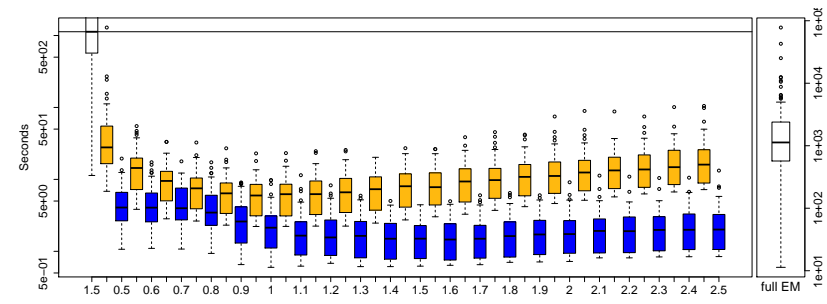

(e) $50 \times 50$ grid with $m=175$

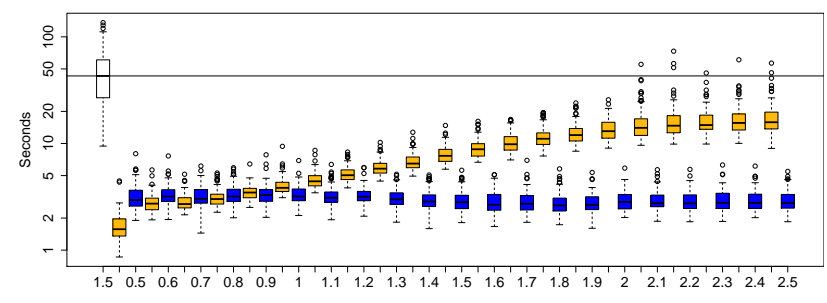

(g) $200 \times 200$ grid with $m=23$

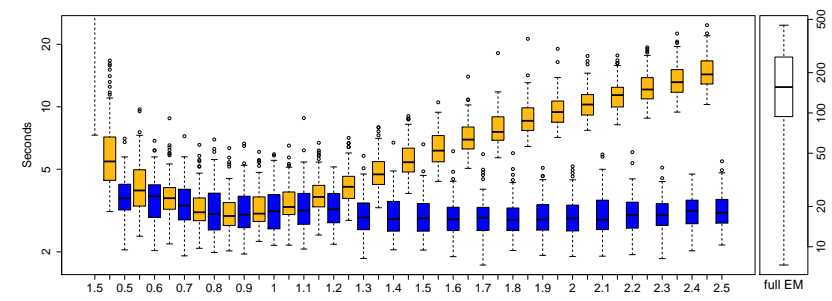

(i) $200 \times 200$ grid with $m=77$

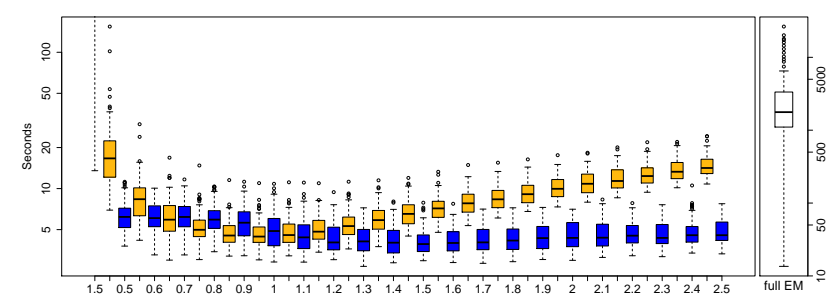

(k) $200 \times 200$ grid with $m=175$

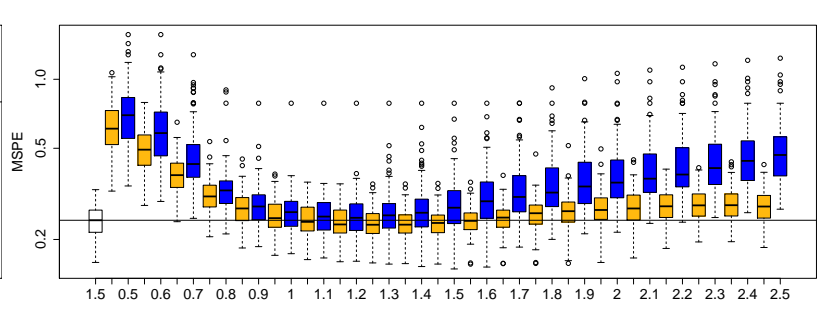

(b) $50 \times 50$ grid with $m=23$

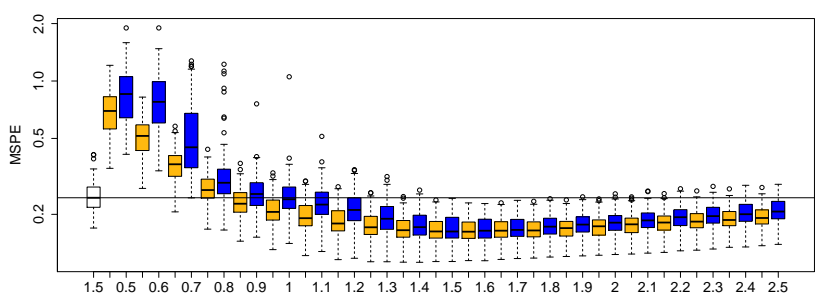

(d) $50 \times 50$ grid with $m=77$

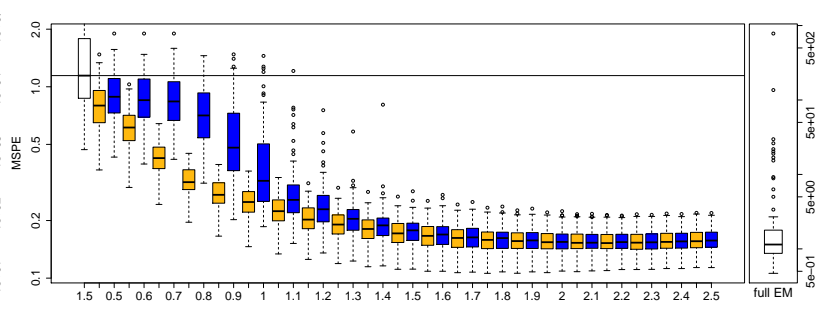

(f) $50 \times 50$ grid with $m=175$

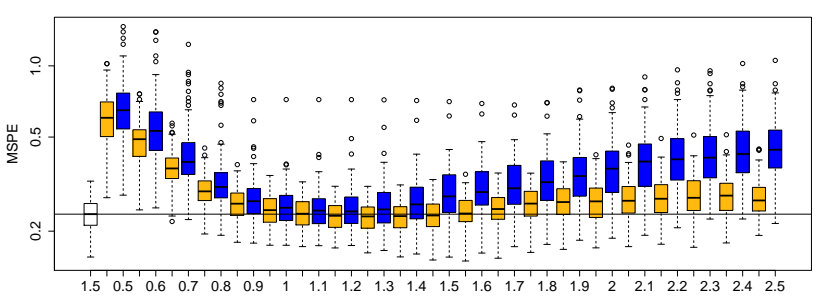

(h) $200 \times 200$ grid with $m=23$

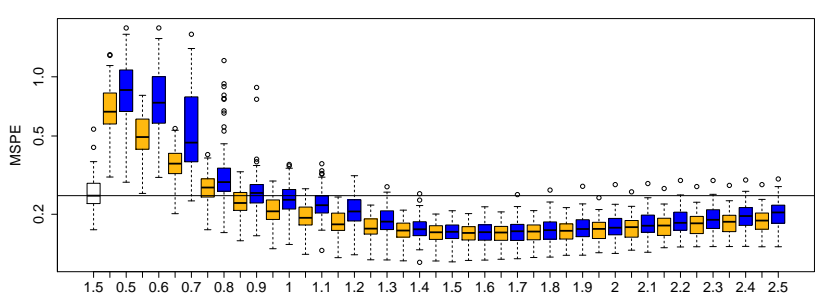

(j) $200 \times 200$ grid with $m=77$

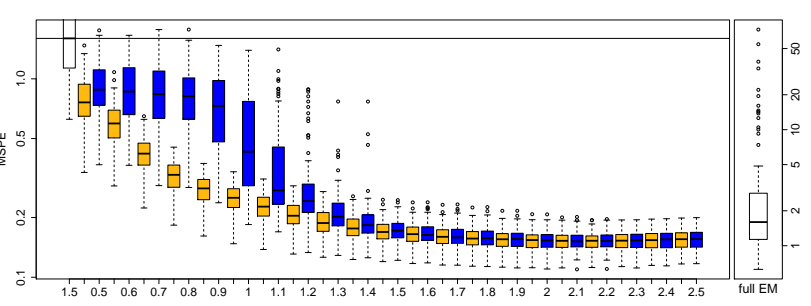

(l) $200 \times 200$ grid with $m=175$

Figure S-8: Seconds (left panel) and Mean Square£@rediction Errors (MSPEs, right panel) for varying bandwidths constants (x-axis) and number of knots $(m)$ for all three estimation methods on both grids when $\nu=1$ and $\sigma^{2}=0.4$. 


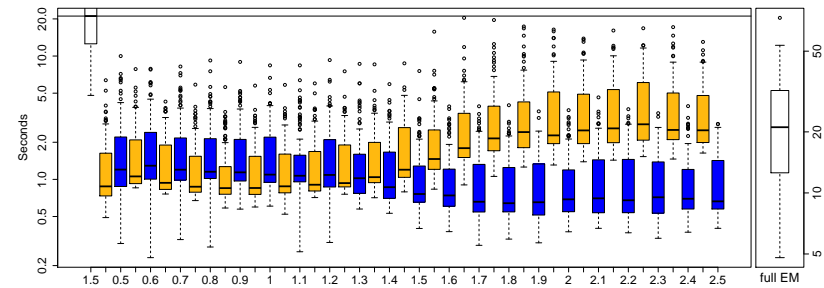

(a) $50 \times 50$ grid with $m=23$

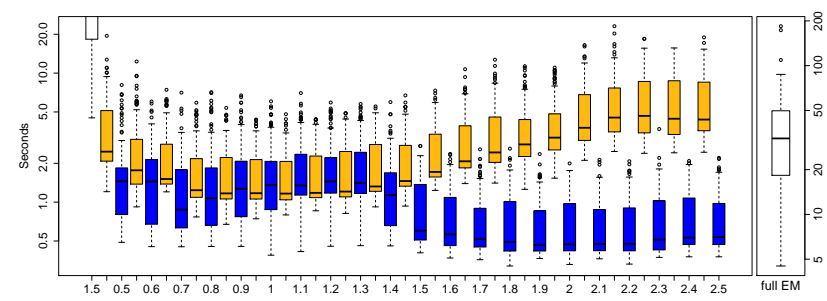

(c) $50 \times 50$ grid with $m=77$

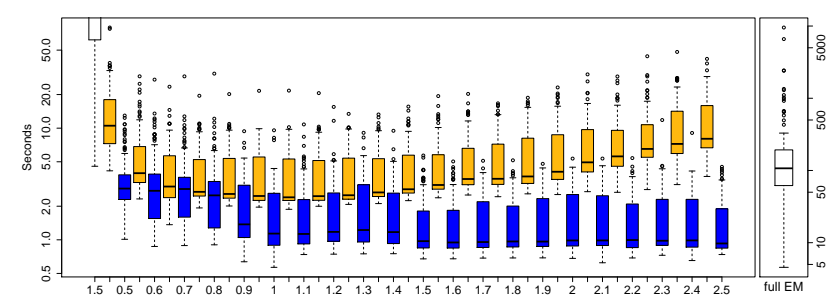

(e) $50 \times 50$ grid with $m=175$

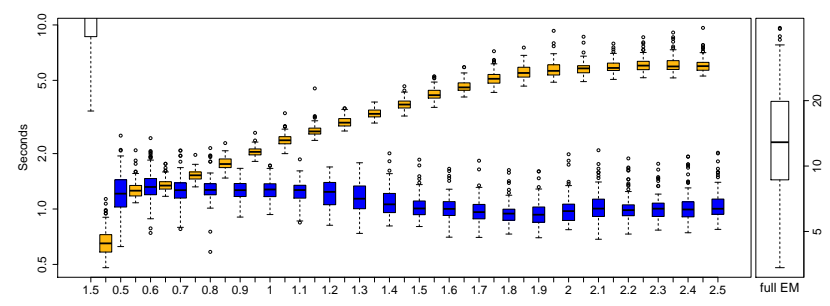

(g) $200 \times 200$ grid with $m=23$

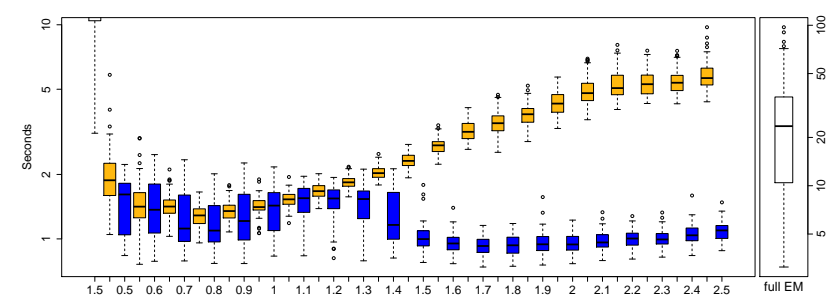

(i) $200 \times 200$ grid with $m=77$

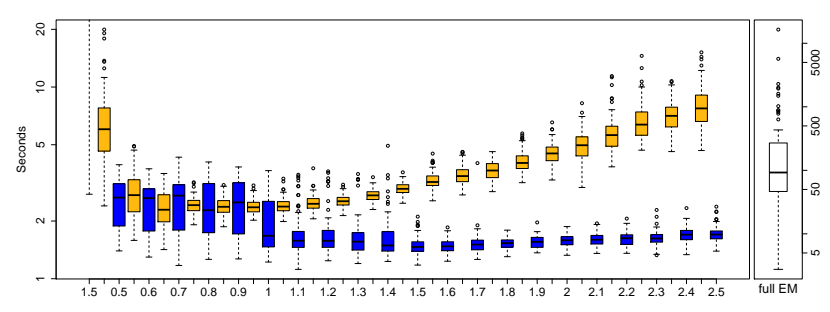

(k) $200 \times 200$ grid with $m=175$

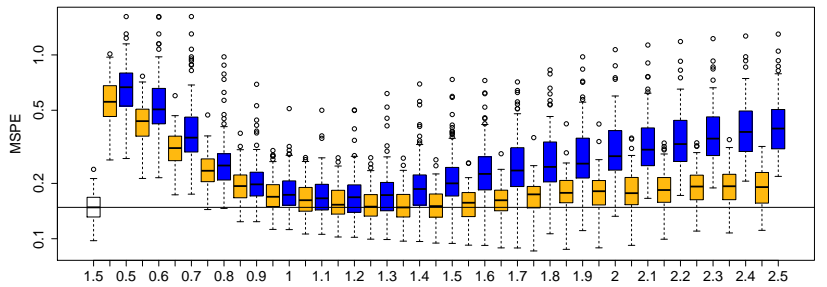

(b) $50 \times 50$ grid with $m=23$

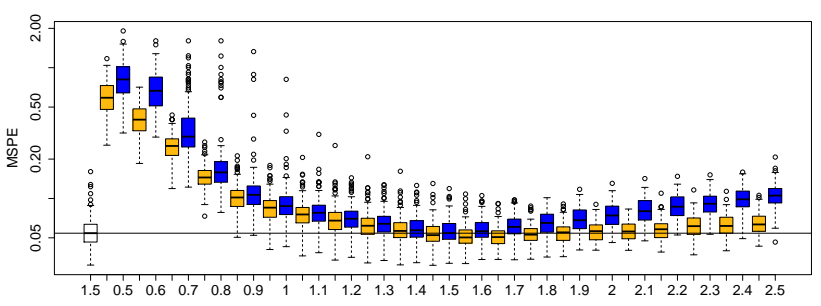

(d) $50 \times 50$ grid with $m=77$

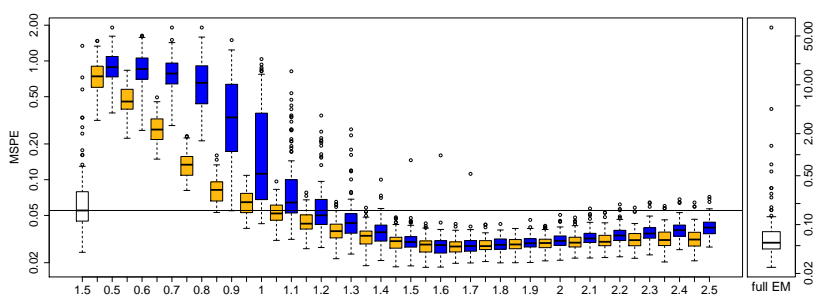

(f) $50 \times 50$ grid with $m=175$

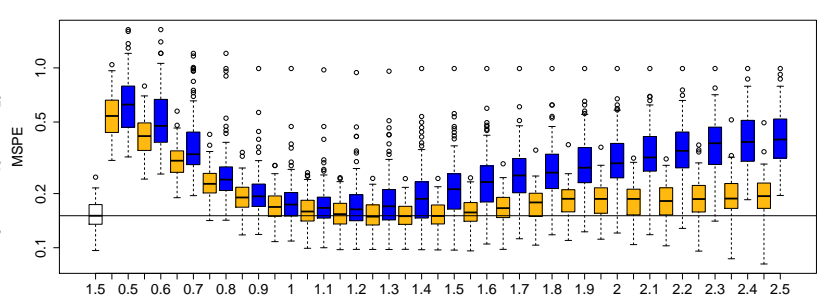

(h) $200 \times 200$ grid with $m=23$

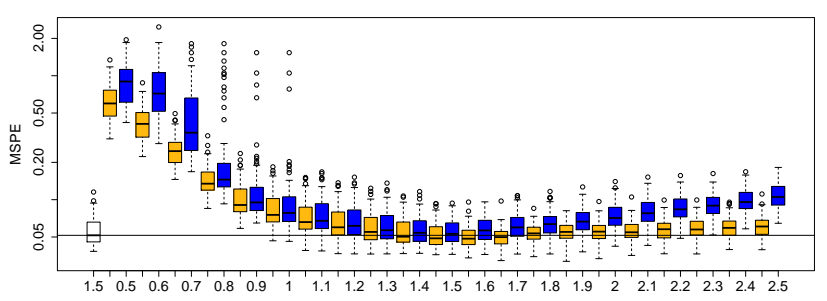

(j) $200 \times 200$ grid with $m=77$

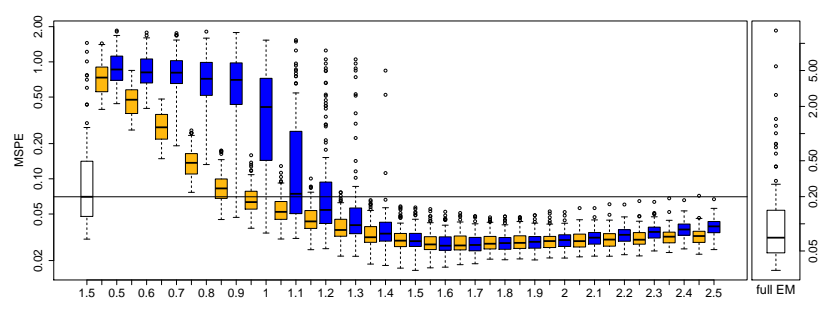

(l) $200 \times 200$ grid with $m=175$

Figure S-9: Seconds (left panel) and Mean Squarełprediction Errors (MSPEs, right panel) for varying bandwidths constants (x-axis) and number of knots $(m)$ for all three estimation methods on both grids when $\nu=1.5$ and $\sigma^{2}=0$. 


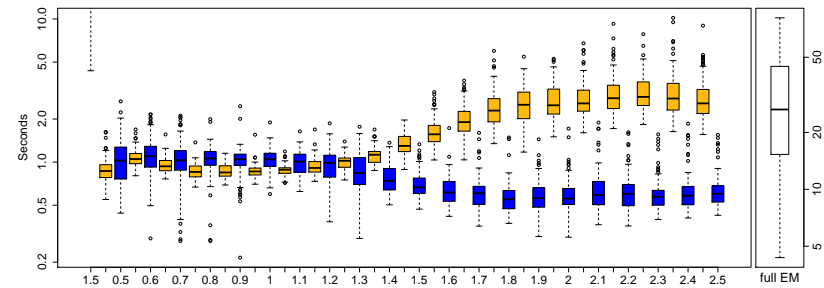

(a) $50 \times 50$ grid with $m=23$

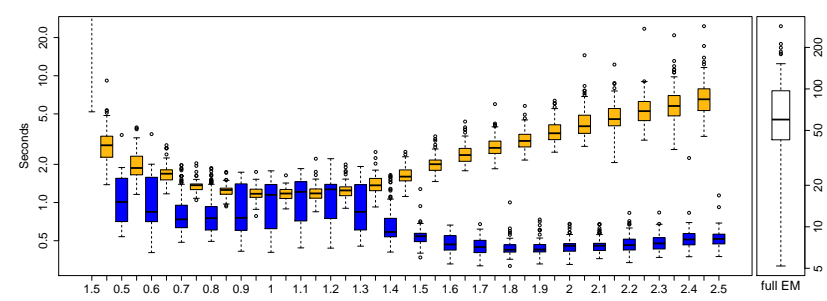

(c) $50 \times 50$ grid with $m=77$

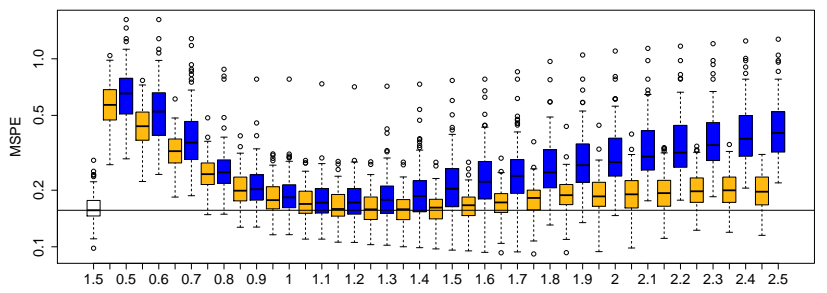

(b) $50 \times 50$ grid with $m=23$

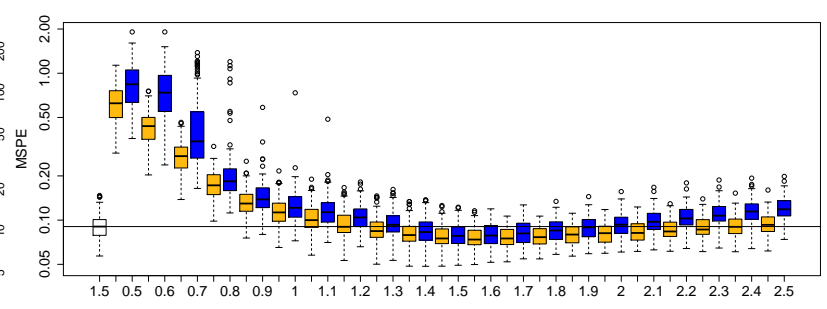

(d) $50 \times 50$ grid with $m=77$

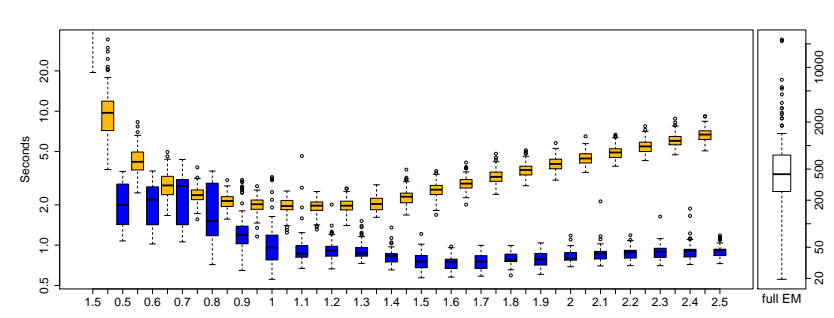

(e) $50 \times 50$ grid with $m=175$

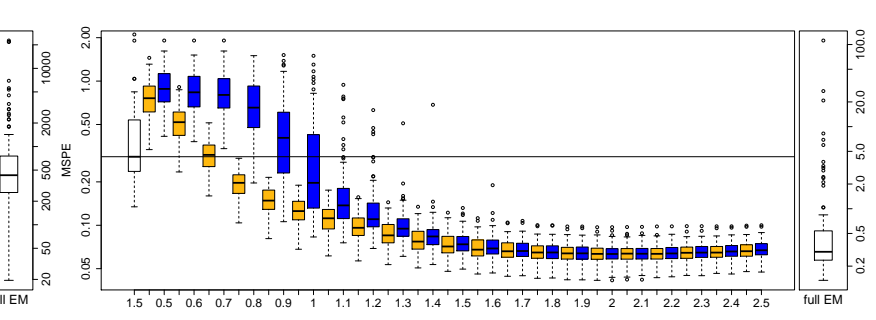

(f) $50 \times 50$ grid with $m=175$

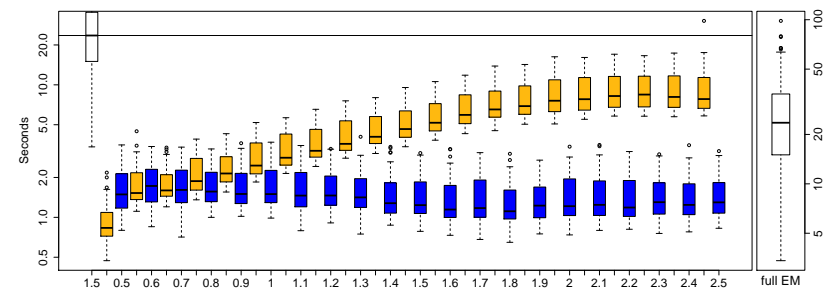

(g) $200 \times 200$ grid with $m=23$

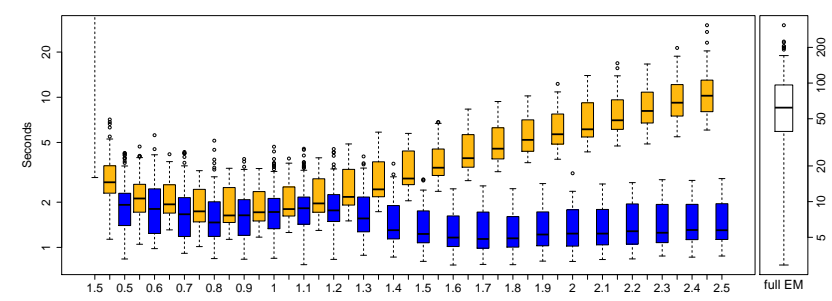

(i) $200 \times 200$ grid with $m=77$

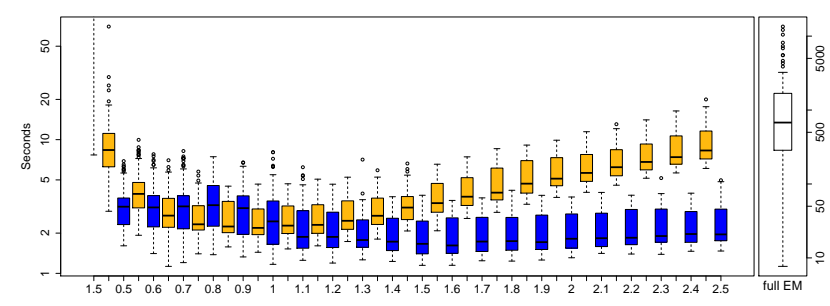

(k) $200 \times 200$ grid with $m=175$

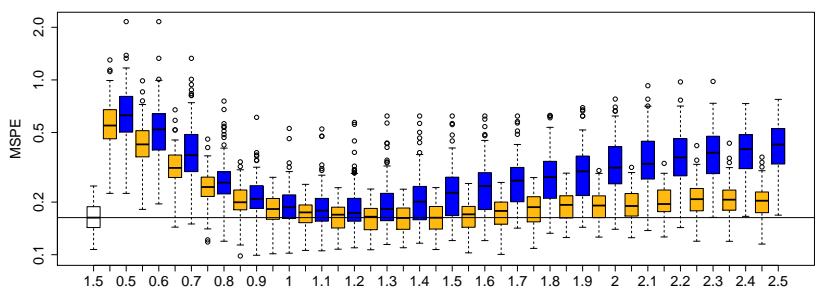

(h) $200 \times 200$ grid with $m=23$

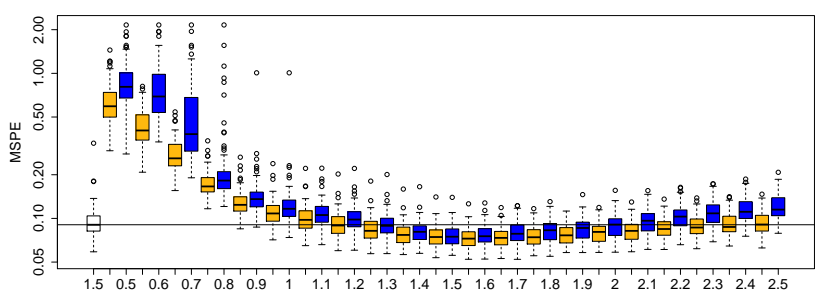

(j) $200 \times 200$ grid with $m=77$

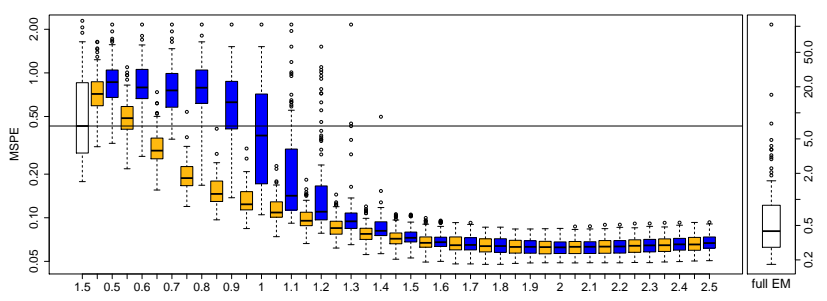

(l) $200 \times 200$ grid with $m=175$

Figure S-10: Seconds (left panel) and Mean Squared Prediction Errors (MSPEs, right panel) for varying bandwidths constants (x-axis) and number of knots $(m)$ for all three estimation methods on both grids when $\nu=1.5$ and $\sigma^{2}=0.1$. 


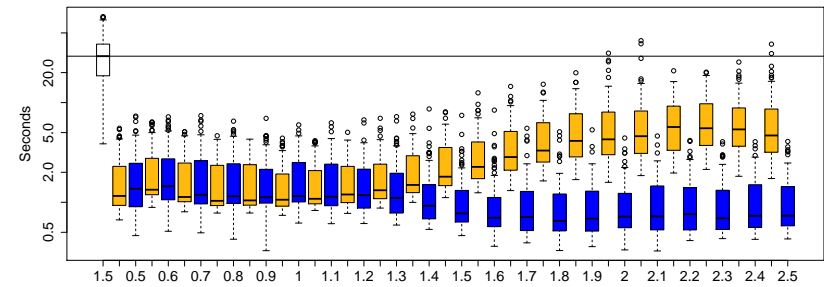

(a) $50 \times 50$ grid with $m=23$

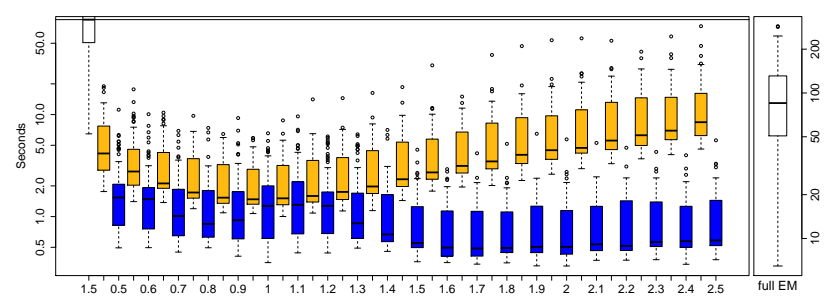

(c) $50 \times 50$ grid with $m=77$

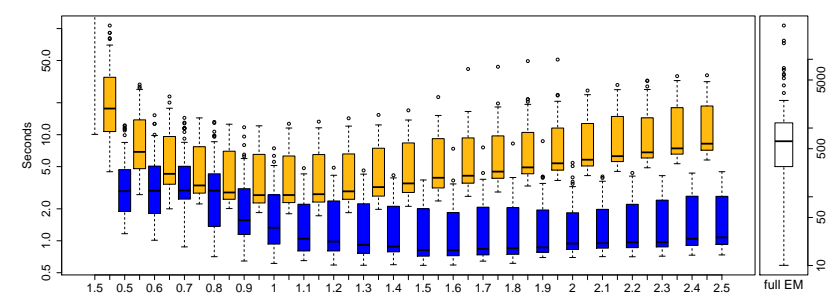

(e) $50 \times 50$ grid with $m=175$

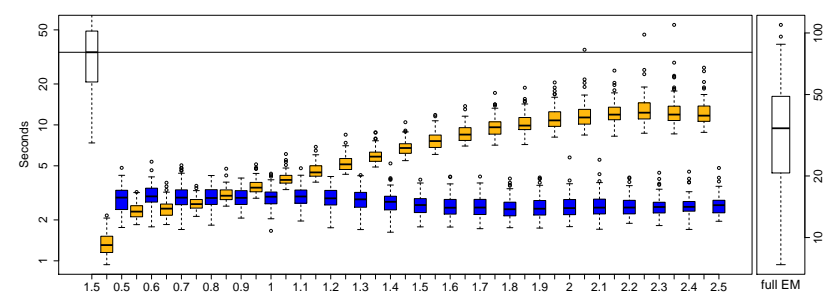

(g) $200 \times 200$ grid with $m=23$

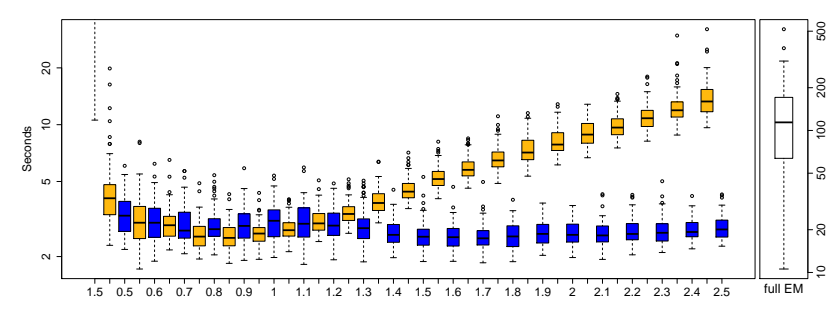

(i) $200 \times 200$ grid with $m=77$

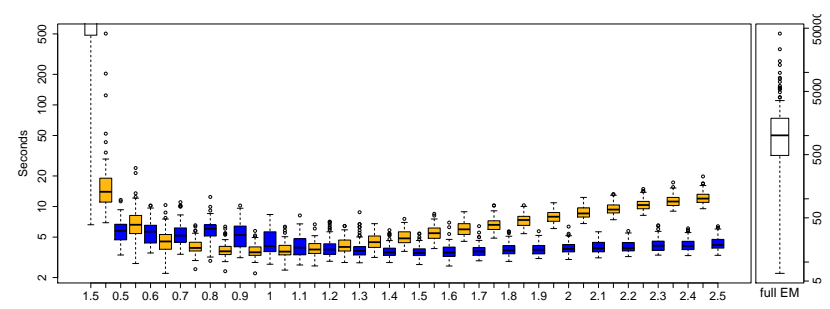

(k) $200 \times 200$ grid with $m=175$

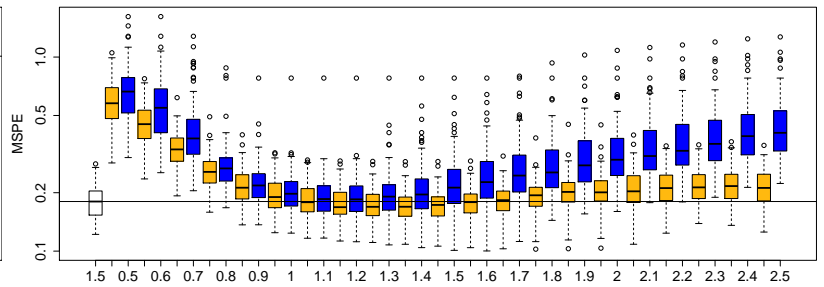

(b) $50 \times 50$ grid with $m=23$

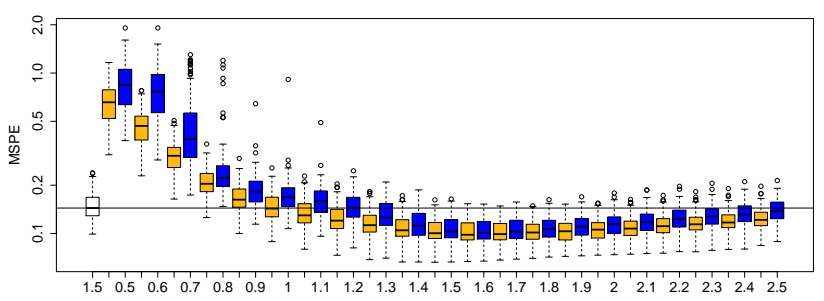

(d) $50 \times 50$ grid with $m=77$

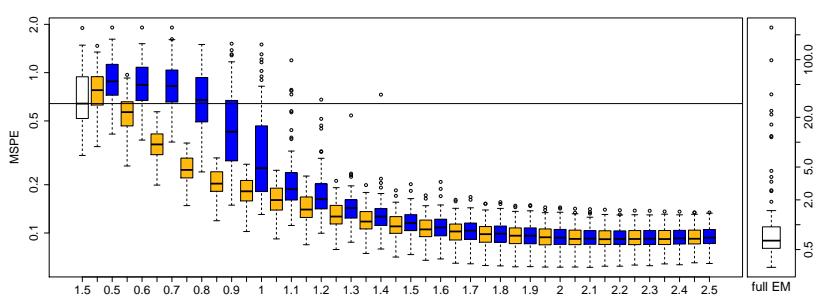

(f) $50 \times 50$ grid with $m=175$

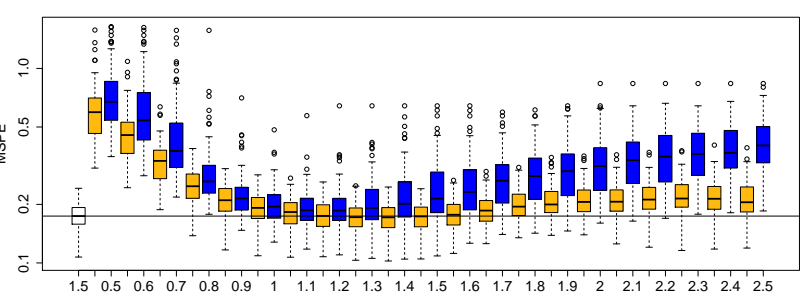

(h) $200 \times 200$ grid with $m=23$

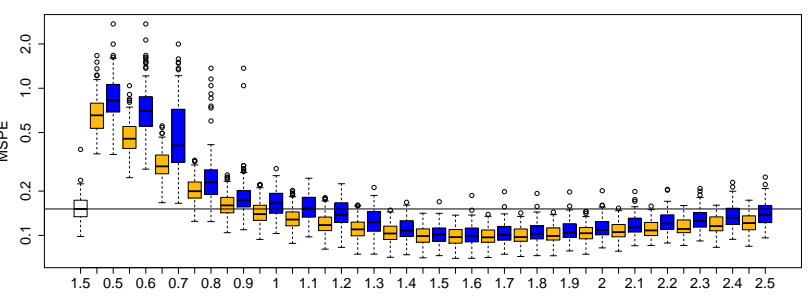

(j) $200 \times 200$ grid with $m=77$

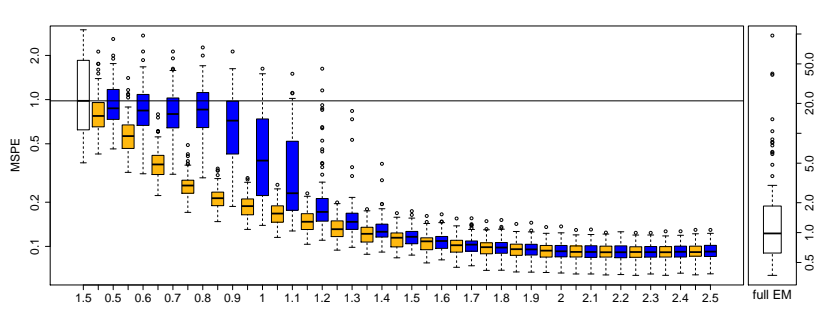

(l) $200 \times 200$ grid with $m=175$

Figure S-11: Seconds (left panel) and Mean Squaregl Prediction Errors (MSPEs, right panel) for varying bandwidths constants (x-axis) and number of knots $(m)$ for all three estimation methods on both grids when $\nu=1.5$ and $\sigma^{2}=0.25$. 


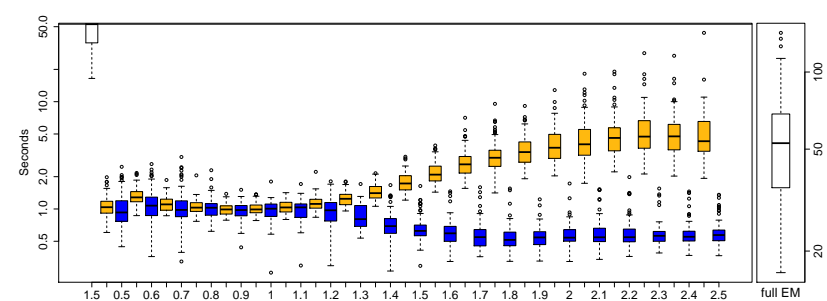

(a) $50 \times 50$ grid with $m=23$

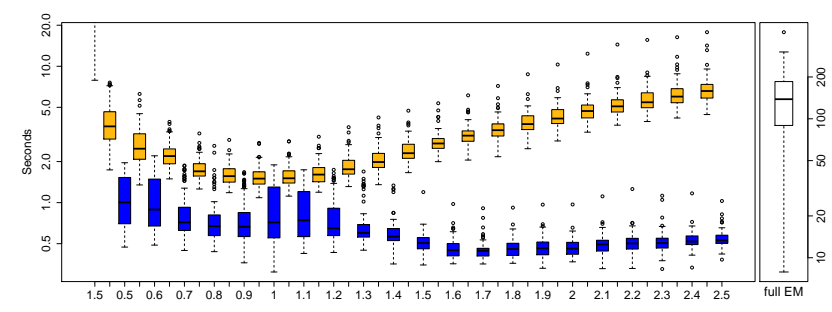

(c) $50 \times 50$ grid with $m=77$

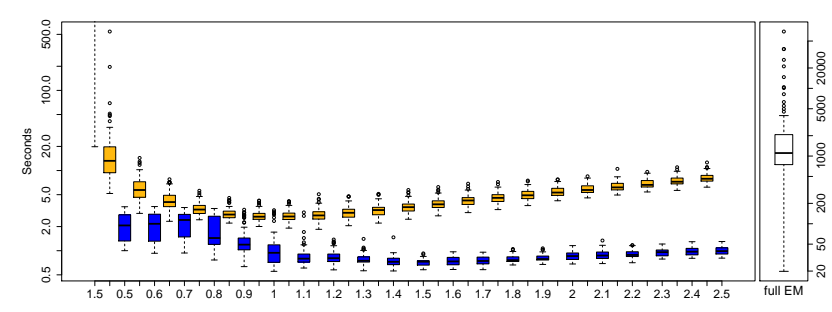

(e) $50 \times 50$ grid with $m=175$

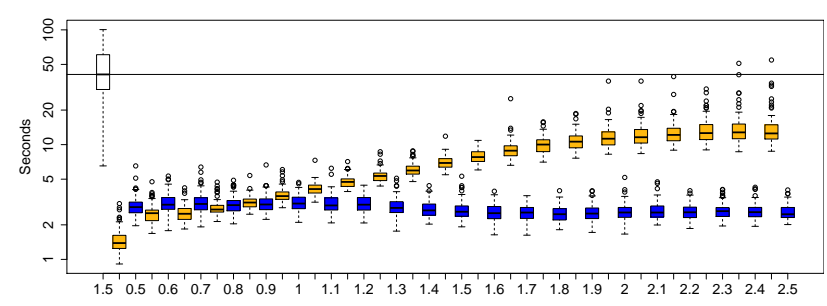

(g) $200 \times 200$ grid with $m=23$

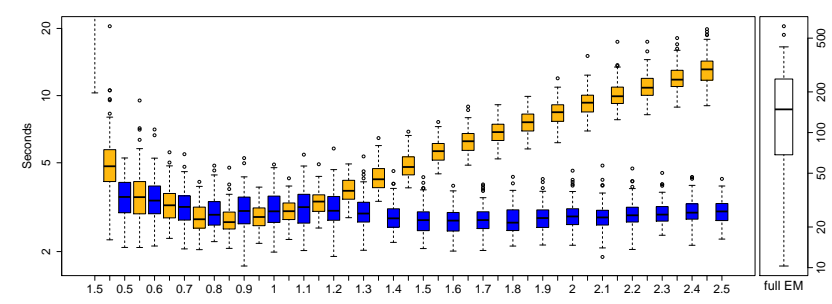

(i) $200 \times 200$ grid with $m=77$

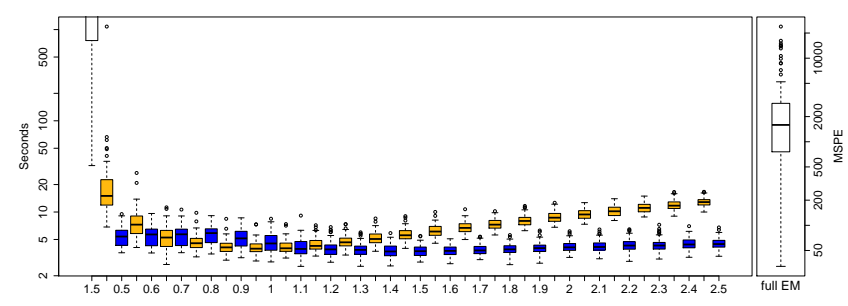

(k) $200 \times 200$ grid with $m=175$

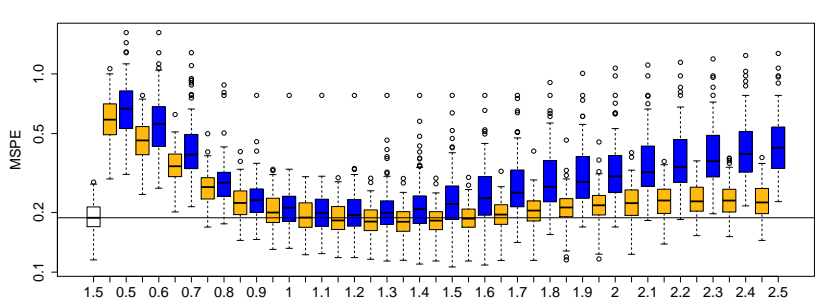

(b) $50 \times 50$ grid with $m=23$

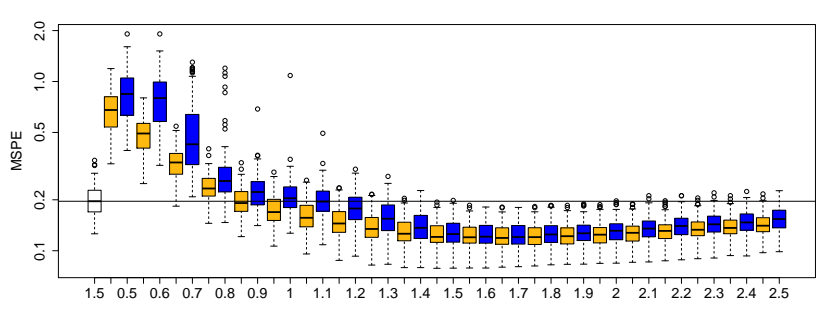

(d) $50 \times 50$ grid with $m=77$

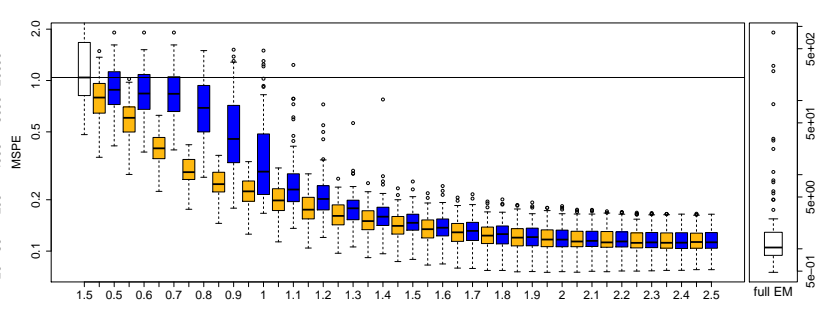

(f) $50 \times 50$ grid with $m=175$

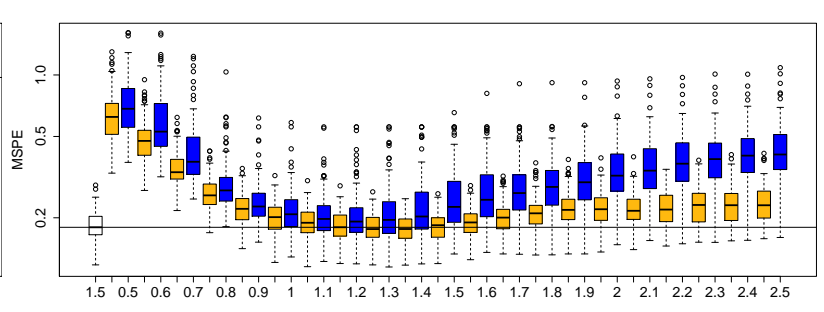

(h) $200 \times 200$ grid with $m=23$

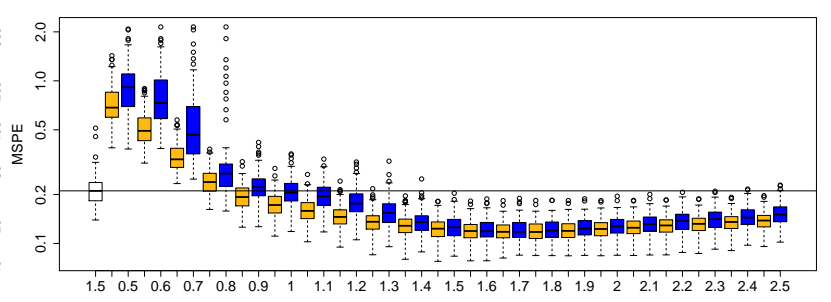

(j) $200 \times 200$ grid with $m=77$

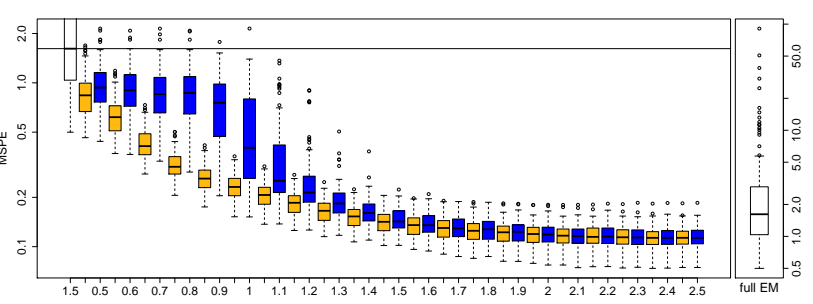

(l) $200 \times 200$ grid with $m=175$

Figure S-12: Seconds (left panel) and Mean Squared Prediction Errors (MSPEs, right panel) for varying bandwidths constants (x-axis) and number of knots $(m)$ for all three estimation methods on both grids when $\nu=1.5$ and $\sigma^{2}=0.4$. 


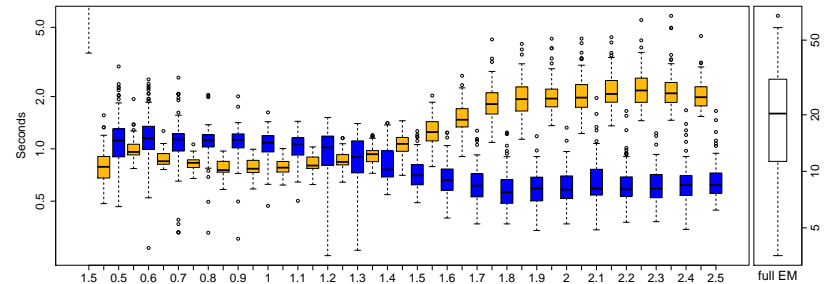

(a) $50 \times 50$ grid with $m=23$

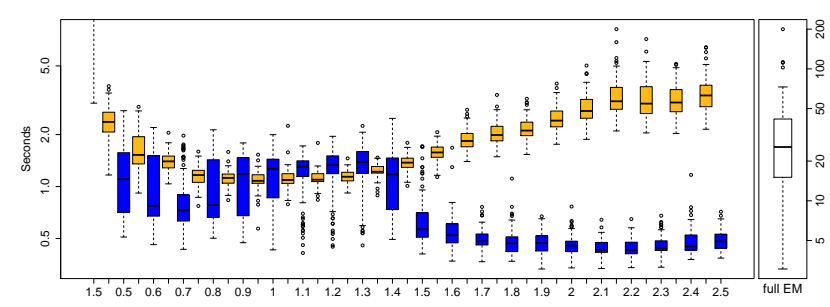

(c) $50 \times 50$ grid with $m=77$

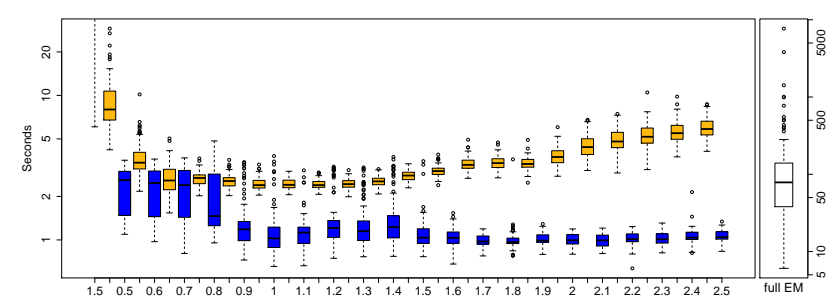

(e) $50 \times 50$ grid with $m=175$

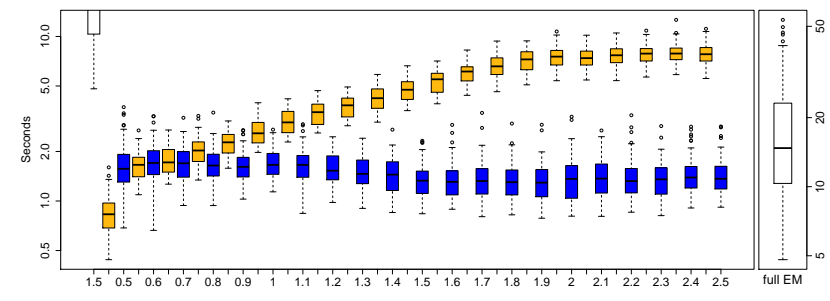

(g) $200 \times 200$ grid with $m=23$

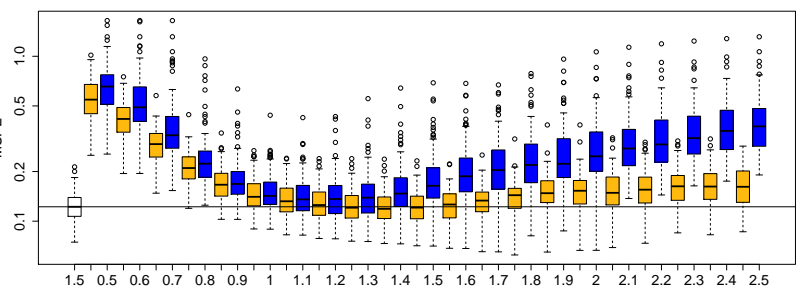

(b) $50 \times 50$ grid with $m=23$

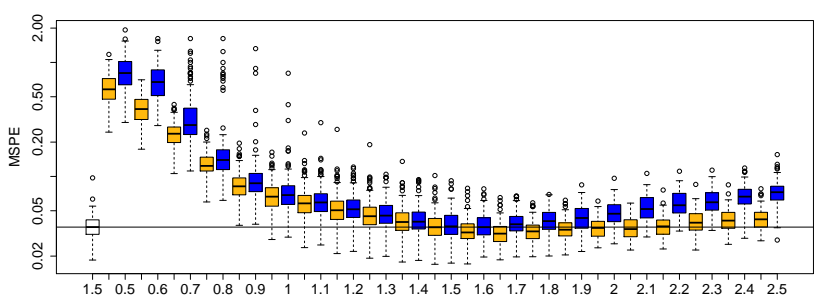

(d) $50 \times 50$ grid with $m=77$

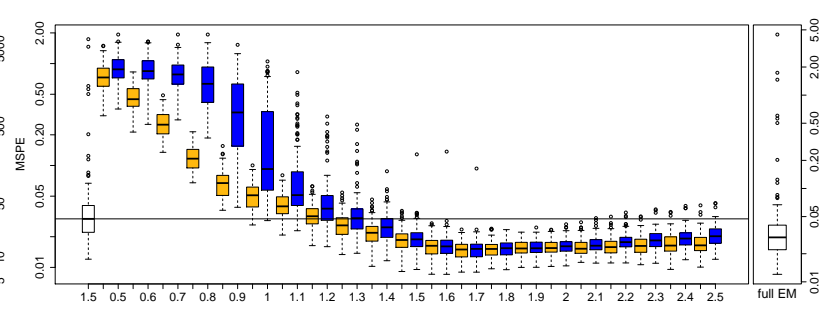

(f) $50 \times 50$ grid with $m=175$

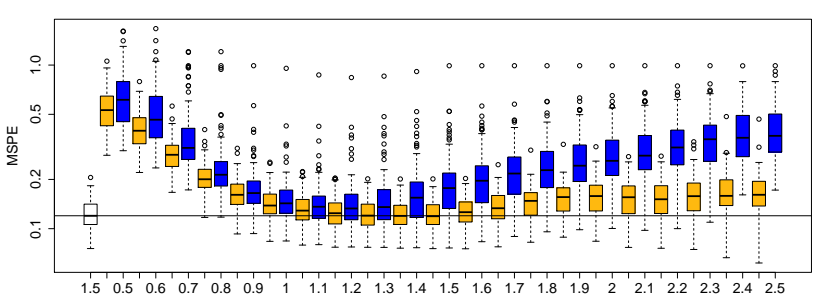

(h) $200 \times 200$ grid with $m=23$

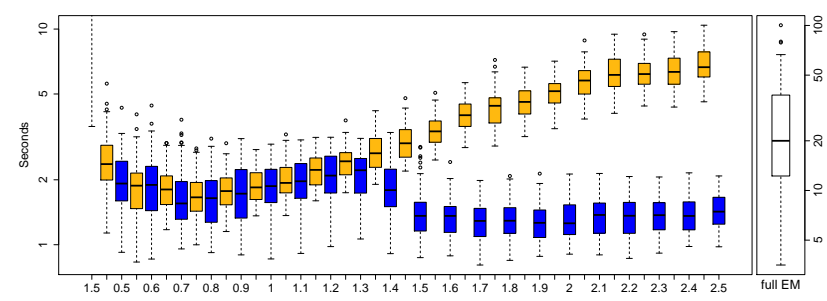

(i) $200 \times 200$ grid with $m=77$

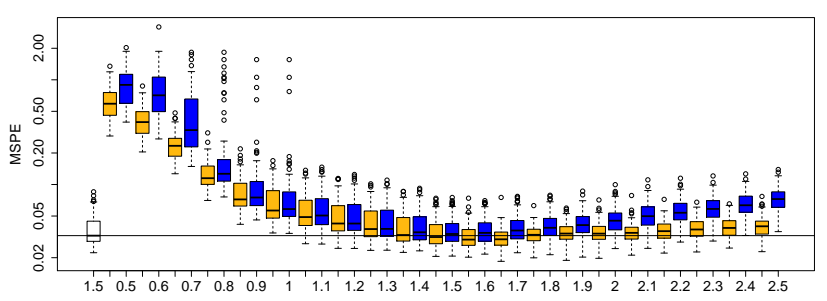

(j) $200 \times 200$ grid with $m=77$

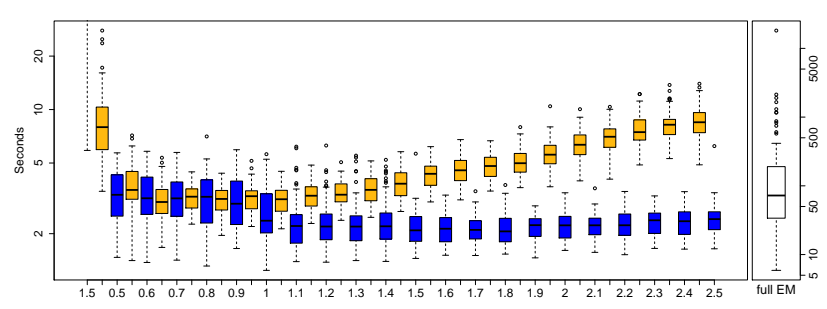

(k) $200 \times 200$ grid with $m=175$

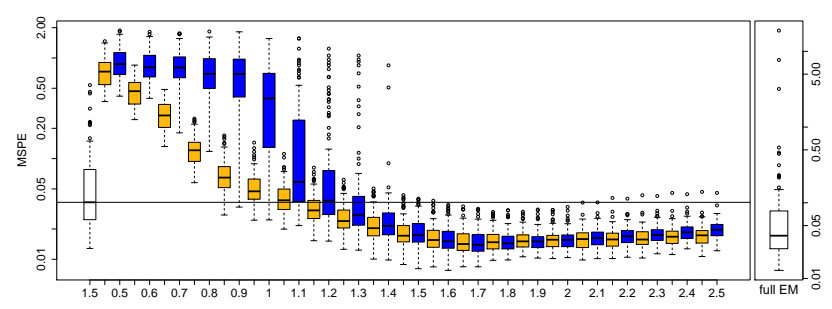

(l) $200 \times 200$ grid with $m=175$

Figure S-13: Seconds (left panel) and Mean Squarefl Prediction Errors (MSPEs, right panel) for varying bandwidths constants (x-axis) and number of knots $(m)$ for all three estimation methods on both grids when $\nu=2$ and $\sigma^{2}=0$. 


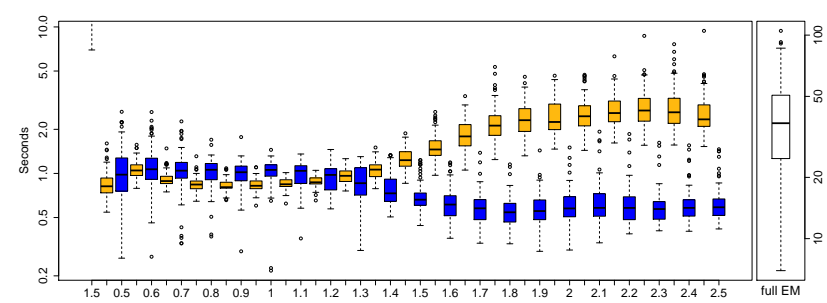

(a) $50 \times 50$ grid with $m=23$

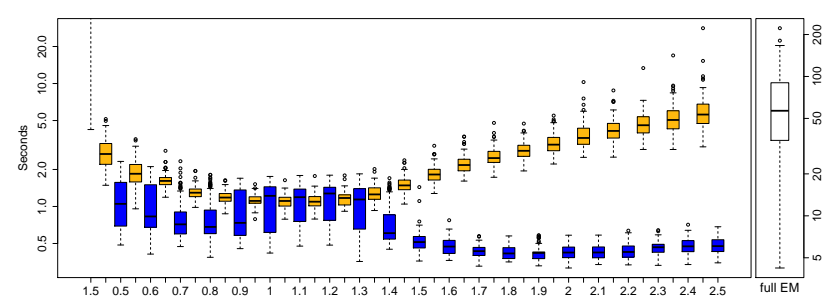

(c) $50 \times 50$ grid with $m=77$

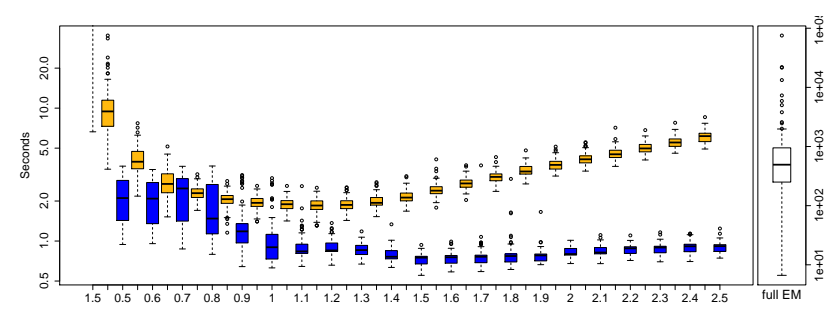

(e) $50 \times 50$ grid with $m=175$

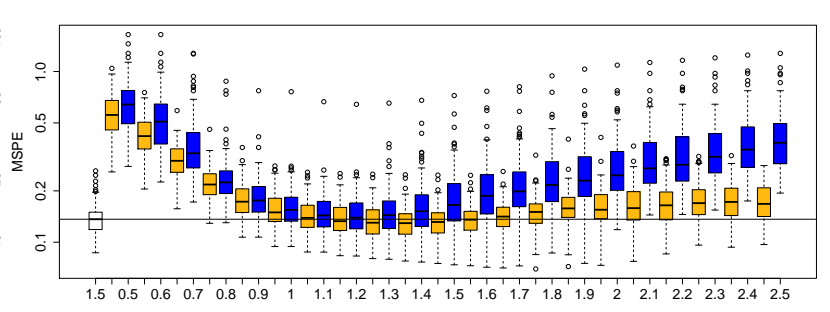

(b) $50 \times 50$ grid with $m=23$

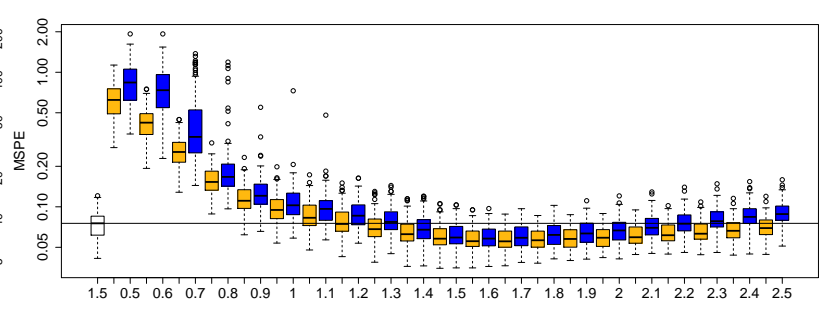

(d) $50 \times 50$ grid with $m=77$

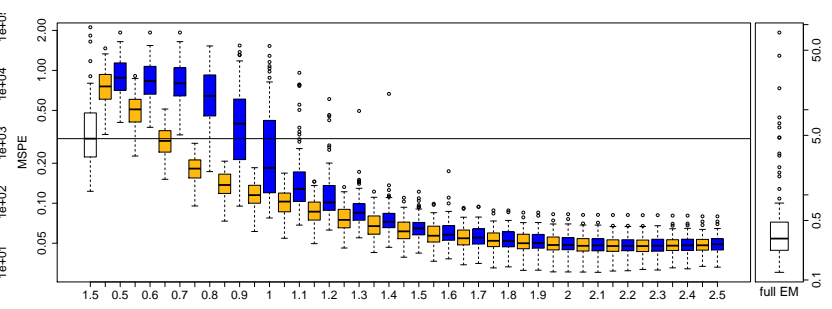

(f) $50 \times 50$ grid with $m=175$

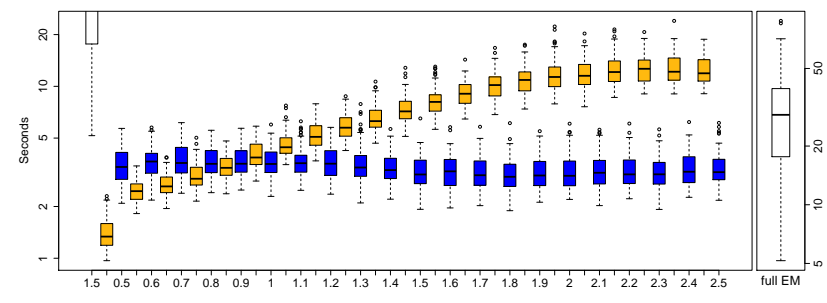

(g) $200 \times 200$ grid with $m=23$

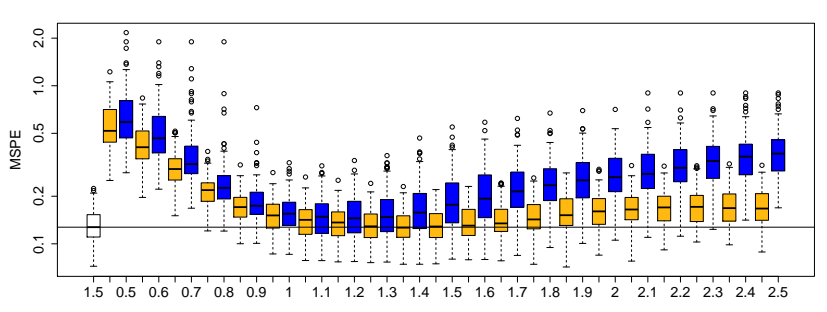

(h) $200 \times 200$ grid with $m=23$

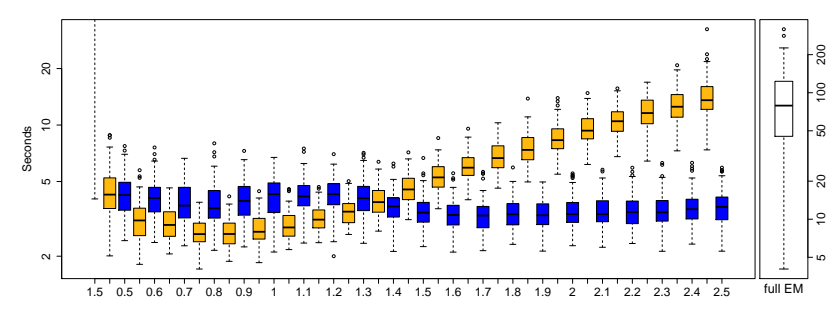

(i) $200 \times 200$ grid with $m=77$

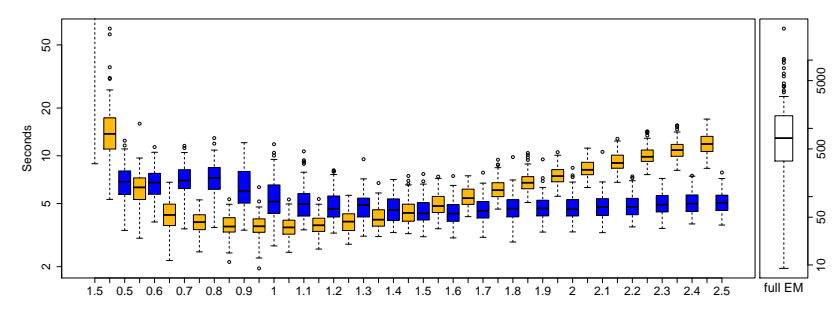

(k) $200 \times 200$ grid with $m=175$

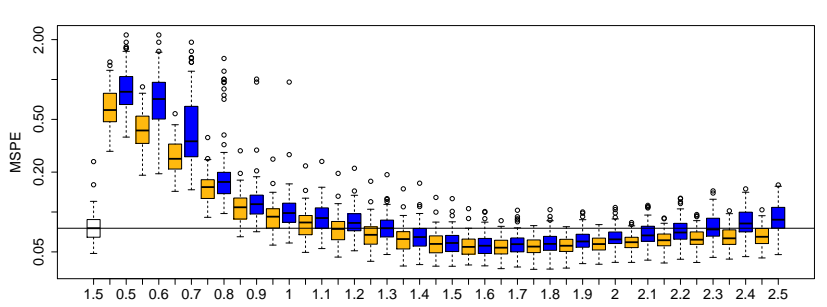

(j) $200 \times 200$ grid with $m=77$

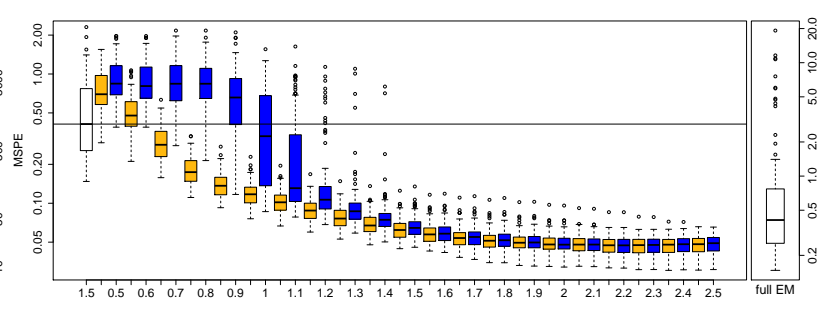

(l) $200 \times 200$ grid with $m=175$

Figure S-14: Seconds (left panel) and Mean Squared Prediction Errors (MSPEs, right panel) for varying bandwidths constants (x-axis) and number of knots $(m)$ for all three estimation methods on both grids when $\nu=2$ and $\sigma^{2}=0.1$. 


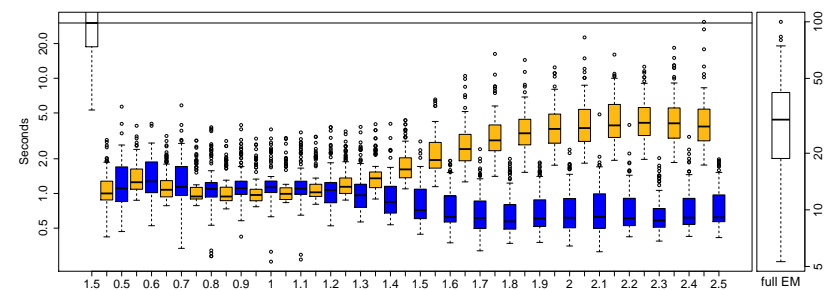

(a) $50 \times 50$ grid with $m=23$

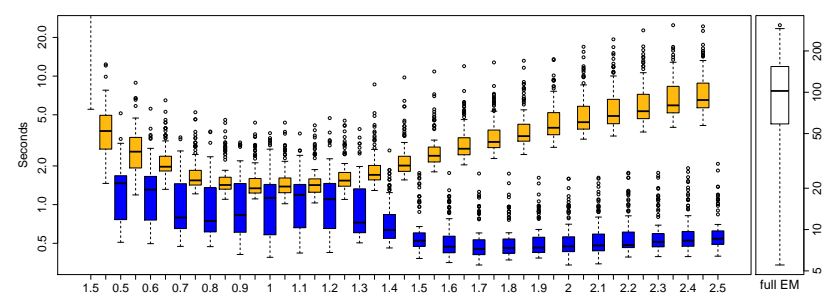

(c) $50 \times 50$ grid with $m=77$

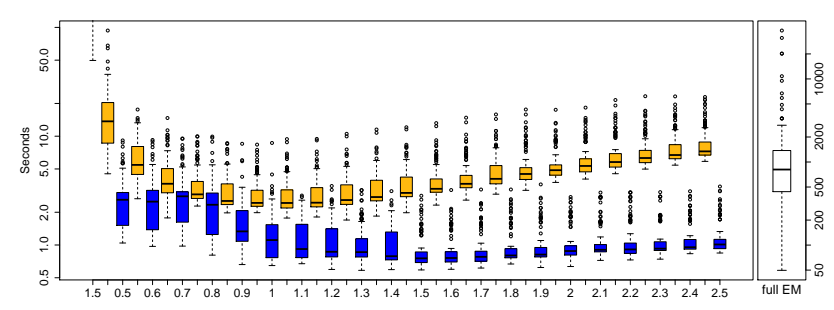

(e) $50 \times 50$ grid with $m=175$

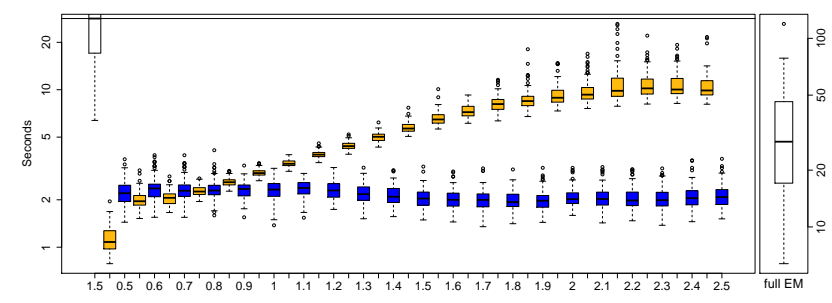

(g) $200 \times 200$ grid with $m=23$

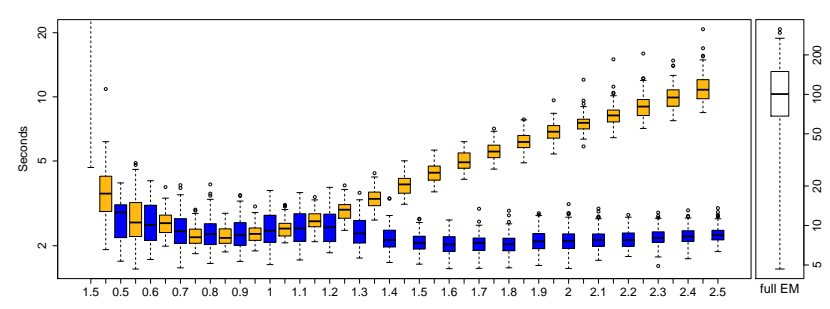

(i) $200 \times 200$ grid with $m=77$

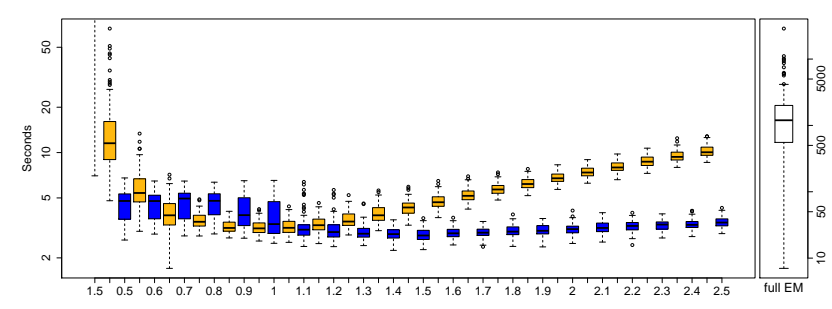

(k) $200 \times 200$ grid with $m=175$

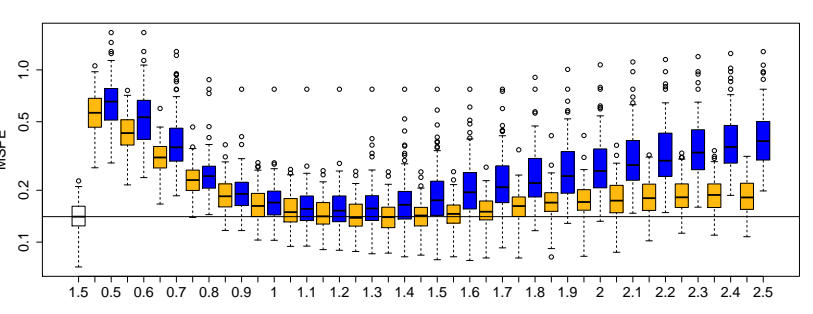

(b) $50 \times 50$ grid with $m=23$

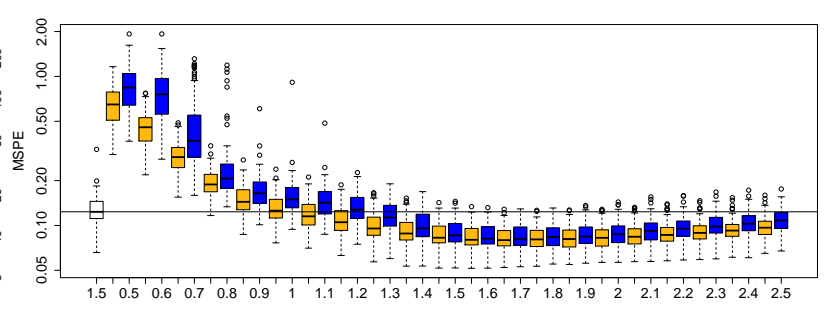

(d) $50 \times 50$ grid with $m=77$

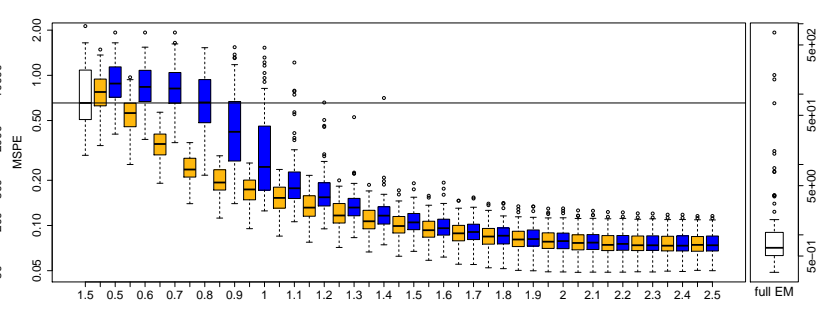

(f) $50 \times 50$ grid with $m=175$

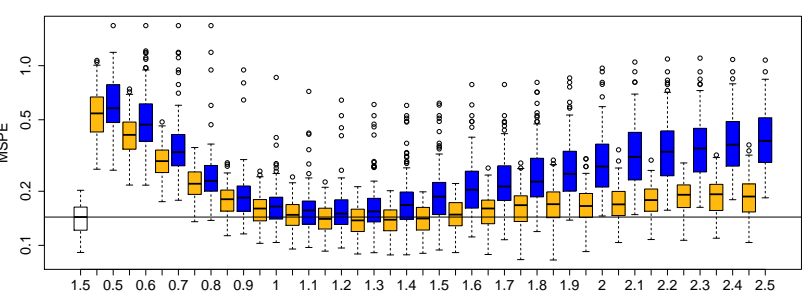

(h) $200 \times 200$ grid with $m=23$

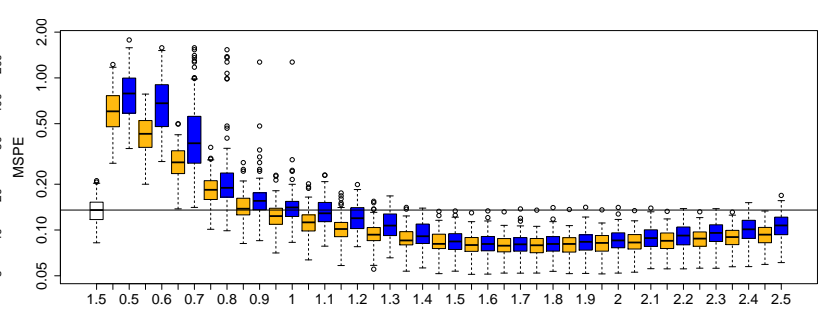

(j) $200 \times 200$ grid with $m=77$

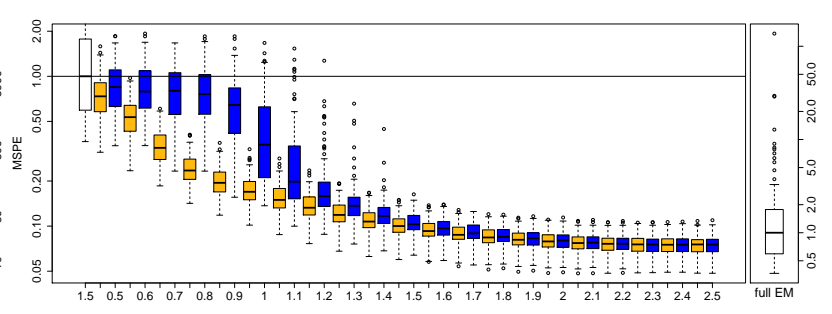

(l) $200 \times 200$ grid with $m=175$

Figure S-15: Seconds (left panel) and Mean Squared Prediction Errors (MSPEs, right panel) for varying bandwidths constants (x-axis) and number of knots $(m)$ for all three estimation methods on both grids when $\nu=2$ and $\sigma^{2}=0.25$. 


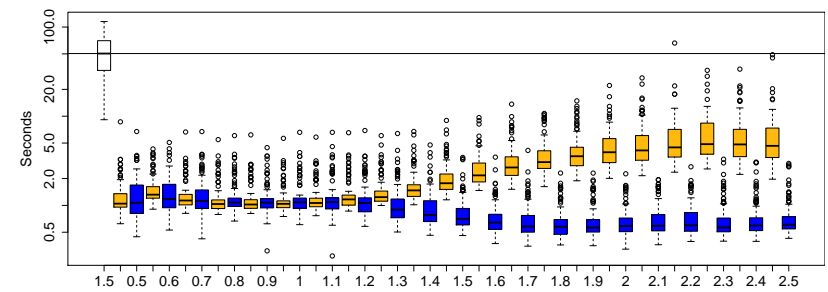

(a) $50 \times 50$ grid with $m=23$

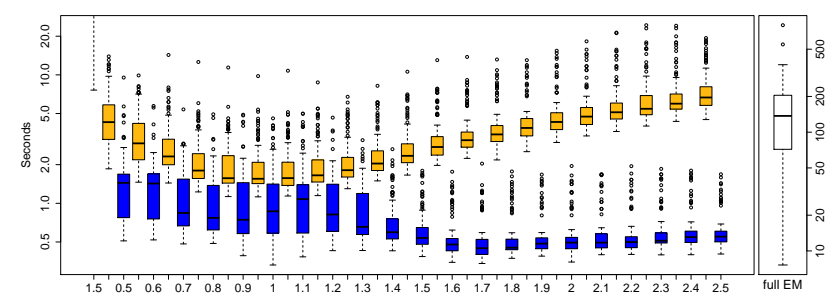

(c) $50 \times 50$ grid with $m=77$

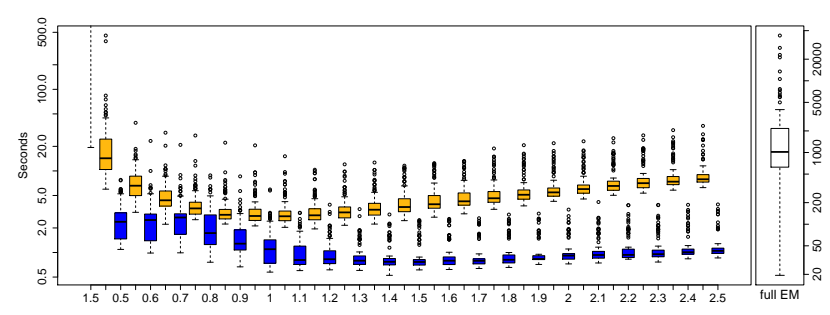

(e) $50 \times 50$ grid with $m=175$

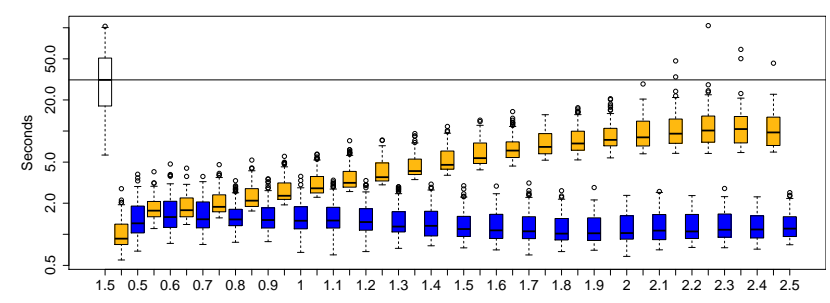

(g) $200 \times 200$ grid with $m=23$

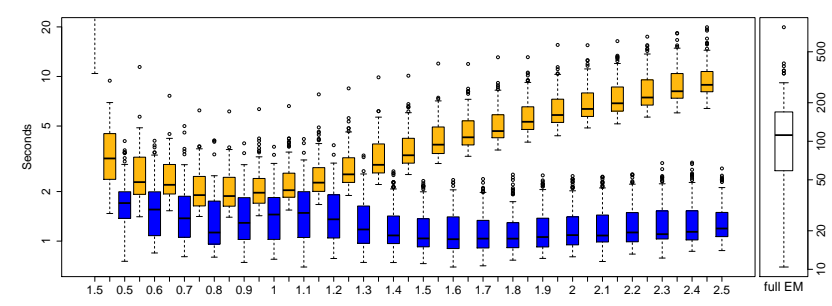

(i) $200 \times 200$ grid with $m=77$

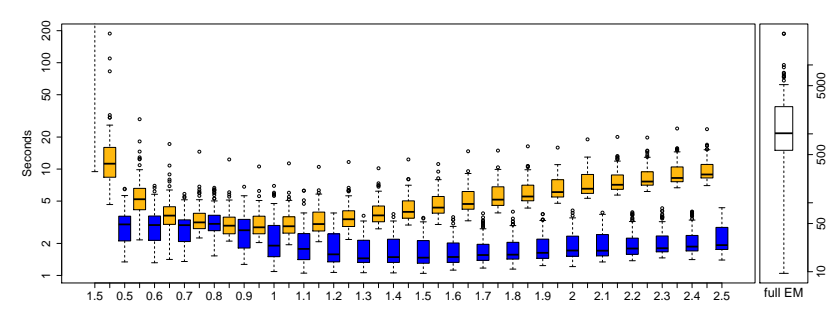

(k) $200 \times 200$ grid with $m=175$

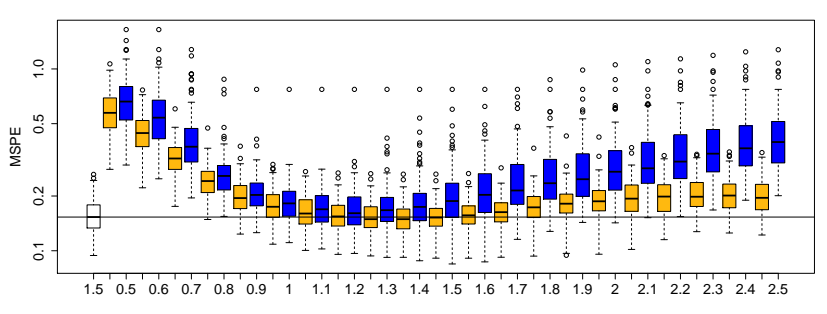

(b) $50 \times 50$ grid with $m=23$

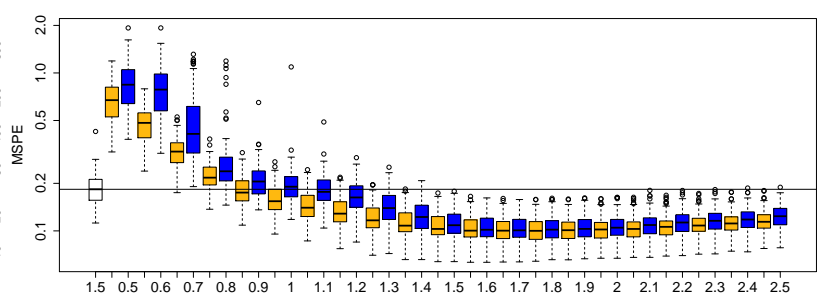

(d) $50 \times 50$ grid with $m=77$

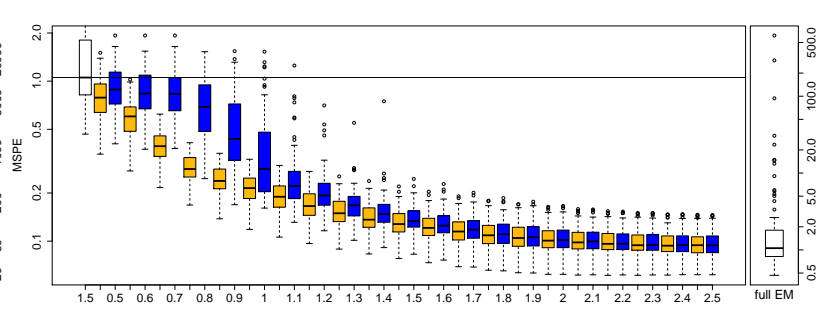

(f) $50 \times 50$ grid with $m=175$

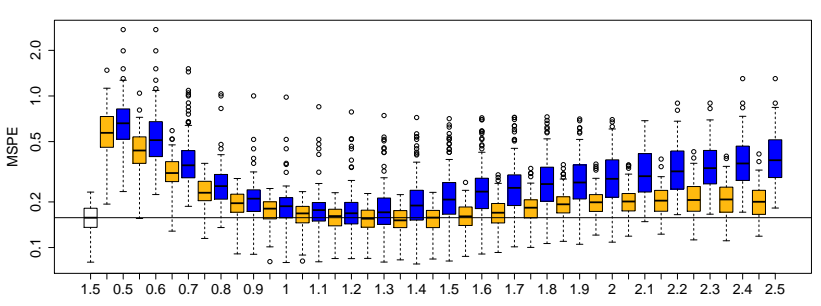

(h) $200 \times 200$ grid with $m=23$

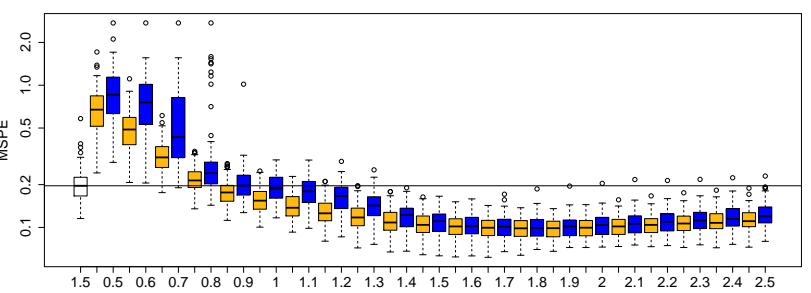

(j) $200 \times 200$ grid with $m=77$

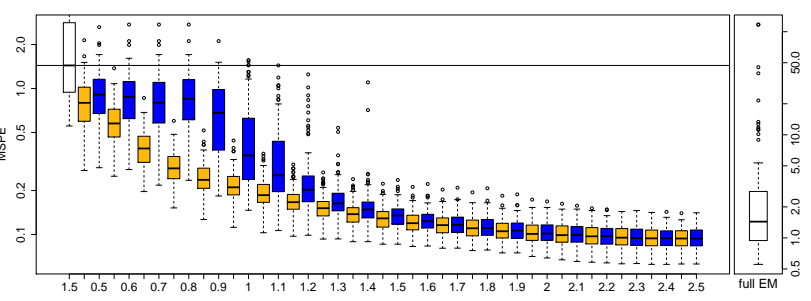

(l) $200 \times 200$ grid with $m=175$

Figure S-16: Seconds (left panel) and Mean Squaregd Prediction Errors (MSPEs, right panel) for varying bandwidths constants (x-axis) and number of knots $(m)$ for all three estimation methods on both grids when $\nu=2$ and $\sigma^{2}=0.4$. 


\section{References}

Bates, D. and Maechler, M. (2015). Matrix: Sparse and Dense Matrix Classes and Methods. R package version 1.2-3.

Davis, T. (2006). Direct Methods for Sparse Linear Systems. Fundamentals of Algorithms. Society for Industrial and Applied Mathematics.

Nychka, D., Furrer, R., Paige, J., and Sain, S. (2015). fields: Tools for Spatial Data. R package version 8.3-5. 